\title{
Direct Catalytic Asymmetric Addition of Acetonitrile to Aldimines
}

\author{
Akira Saito, Naoya Kumagai,* Masakatsu Shibasaki* \\ Institute of Microbial Chemistry (BIKAKEN), Tokyo, Japan \\ nkumagai@bikaken.or.jp, mshibasa@bikaken.or.jp
}

\section{General Methods}

1-1. Reactions and purifications

1-2. Characterizations

$1-3$. Solvents and reagents

2. Preparation of Materials

2-1. Preparation of imines

2-2. Synthesis of chiral Ni(II) complex

3. Catalytic Asymmetric Addition to Imines 5

3-1. Brief ligand screening

3-2. General procedure for direct catalytic asymmetric addition to imines

3-3. Characterization of Mannich adducts

3-4. Large-scale synthesis

3-5. Determination of absolute configuration of the products 


\section{General Methods}

\section{1-1. Reactions and purifications}

Unless otherwise noted, all reactions were carried out in an oven-dried glassware fitted with a 3-way glass stopcock under an argon atmosphere with magnetically stirred chips. All work-up and purification procedures were carried out with reagent-grade solvents under ambient atmosphere. Thin layer chromatography (TLC) was performed on Merck TLC plates $(0.25 \mathrm{~mm})$ pre-coated with silica gel $60 \mathrm{~F} 254$ and visualized by UV quenching and staining with $\mathrm{KMnO}_{4}$ or anisaldehyde solution. Flash column chromatography was performed with $\mathrm{SiO}_{2}$ Merck 60 (230-400 mesh), or on a Teledyne CombiFlash Rf 200.

\section{1-2. Characterizations}

Infrared (IR) spectra were recorded on a HORIBA FT210 Fourier transform infrared spectrophotometer. NMR spectra were recorded on a Bruker AVANCE III HD400 or a Bruker JEOL ECS-600. Chemical shifts $(\delta)$ are given in ppm relative to residual solvent peaks. Data for ${ }^{1} \mathrm{H}$ NMR are reported as follows: chemical shift (multiplicity, coupling constants where applicable, number of hydrogens). Abbreviations are as follows: $s$ (singlet), $d$ (doublet), $t$ (triplet), dd (doublet of doublet), dt (doublet of triplet), ddd (doublet of doublet of doublet), q (quartet), m (multiplet), br (broad). For ${ }^{19} \mathrm{~F}$ NMR, chemical shifts were reported in the scale relative to $\mathrm{PhCF}_{3}\left(\delta-62.7680 \mathrm{ppm}\right.$ in $\left.\mathrm{CDCl}_{3}\right)$ as an external reference. For ${ }^{31} \mathrm{P}$ $\mathrm{NMR}$, chemical shifts were reported in the scale relative to phosphoric acid $\left(\delta 0.000 \mathrm{ppm}\right.$ in $\left.\mathrm{D}_{2} \mathrm{O}\right)$ as an external reference. ${ }^{1}$ High performance liquid chromatography (HPLC) analysis was performed on a JASCO HPLC system equipped with Daicel chiral-stationary-phase columns. Optical rotation was measured using a $1 \mathrm{~mL}$ cell with a $1.0 \mathrm{dm}$ path length on a JASCO polarimeter P-1030. High-resolution mass spectra were measured on a Thermo Fisher Scientific LTQ Orbitrap XL (ESI) or JEOL JMS-T100GCv (EI).

\section{1-3. Solvents and reagents}

Anhydrous $\mathrm{THF}, \mathrm{CH}_{3} \mathrm{CN}$, and $\mathrm{CH}_{2} \mathrm{Cl}_{2}$ were purified by passing through a solvent purification system (Glass Contour). $N, N$-dimethylacetamide was purchased from Aldrich (anhydrous). All reagents were used as supplied by commercial vendors or prepared by the method described in the corresponding references. 


\section{Preparation of Materials}

\section{2-1. Preparation of imines}

All N-Dpp imines were known compounds and were prepared according to the literature procedure. ${ }^{2}$ The compounds obtained by flash column chromatography were recrystallized in $\mathrm{CH}_{2} \mathrm{Cl}_{2} / n$-hexane prior to use.

\section{2-2. Synthesis of chiral Ni(II) complex}

\section{$<$ imidazolium salt synthesis>}

Bisimidazolium salts S1a, S1b, and S1d were synthesized according to the known procedure. ${ }^{3}$

S1c was synthesized according to the procedure described below.

(5S,5'S)-2,2'-(1,3-Phenylene)bis(5-phenyl-5,6-dihydro-8H-[1,2,4]triazolo[3,4-c][1,4]oxazin-2-

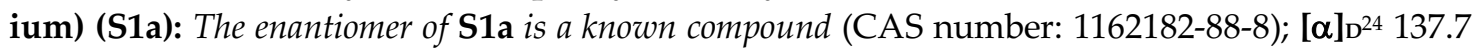
(c $\left.0.50, \mathrm{CH}_{3} \mathrm{OH}\right)$.

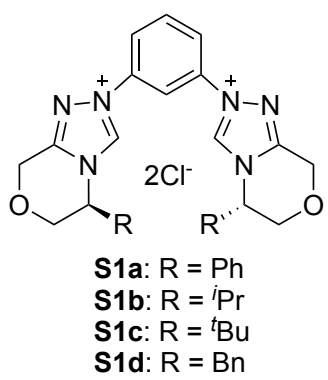

(5S,5'S)-2,2'-(1,3-Phenylene)bis(5-(tert-butyl)-5,6-dihydro-8H-[1,2,4]triazolo[3,4-c][1,4]oxazin-2-ium) dichloride (S1c):

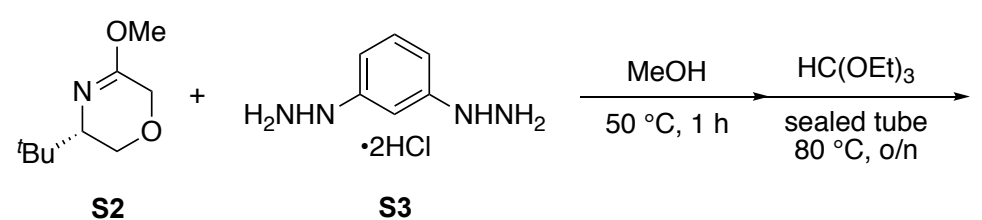<smiles></smiles>

To a solution of $\mathbf{S 3}^{4}(305 \mathrm{mg}, 1.44 \mathrm{mmol})$ in $\mathrm{MeOH}(5.0 \mathrm{~mL})$ was added a solution of $\mathbf{S 2}^{5}$ (495 mg, $\left.2.89 \mathrm{mmol}\right) \mathrm{in} \mathrm{MeOH}$ $(5.0 \mathrm{~mL})$ at $50{ }^{\circ} \mathrm{C}$. After stirring for $1 \mathrm{~h}$, the solution was transferred to a sealed tube, triethyl orthoformate $(2.4 \mathrm{~mL}, 14$ $\mathrm{mmol}$ ) was added, and stirred overnight at $80^{\circ} \mathrm{C}$. After volatiles were removed in vacuo, the residue was purified by silica gel column chromatography $\left(\mathrm{CHCl}_{3} / \mathrm{MeOH}=80 / 20-60 / 40\right)$ to afford $\mathrm{S1c}$ as a pink solid $(491 \mathrm{mg}, 55 \%)$.

m.p. 209-211 ${ }^{\circ} \mathrm{C}$; IR $\left(\mathrm{CHCl}_{3}\right)$ 3398, 2967, 1615, 1580, 1528, 1473, 1398, 1226, $1119 \mathrm{~cm}^{-1}$; ${ }^{1} \mathbf{H}$ NMR $(400 \mathrm{MHz}, \mathrm{CD} 3 \mathrm{CN}) \delta 12.62$ $(\mathrm{d}, J=1.3 \mathrm{~Hz}, 2 \mathrm{H}), 9.98(\mathrm{~s}, 1 \mathrm{H}), 8.20(\mathrm{dd}, J=8.3,2.1 \mathrm{~Hz}, 2 \mathrm{H}), 8.00-7.86(\mathrm{~m}, 1 \mathrm{H}), 5.27(\mathrm{~d}, J=16.9 \mathrm{~Hz}, 2 \mathrm{H}), 4.99(\mathrm{dd}, J=16.9$, $0.7 \mathrm{~Hz}, 2 \mathrm{H}), 4.61-4.46(\mathrm{~m}, 2 \mathrm{H}), 4.36(\mathrm{~d}, J=3.6 \mathrm{~Hz}, 2 \mathrm{H}), 3.93(\mathrm{dd}, J=13.3,3.7 \mathrm{~Hz}, 2 \mathrm{H}), 1.19(\mathrm{~s}, 18 \mathrm{H}) ;{ }^{13} \mathrm{C} \mathrm{NMR}(100 \mathrm{MHz}$, $\mathrm{CD}_{3} \mathrm{CN}$ ) $\delta 151.7,144.1,137.2,133.4,122.7,114.0,65.3,65.0,62.3,35.9,27.4 ;$ HRMS (ESI) $m / z$ calc'd for $\mathrm{C}_{24} \mathrm{H}_{33} \mathrm{~N}_{6} \mathrm{O}_{2}$ [M $-2 \mathrm{Cl}$

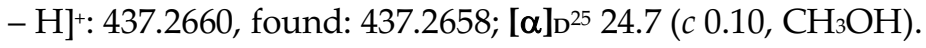

$<$ General procedure for preparation of pincer Ni(II) complexes S4a-d $>$<smiles></smiles>
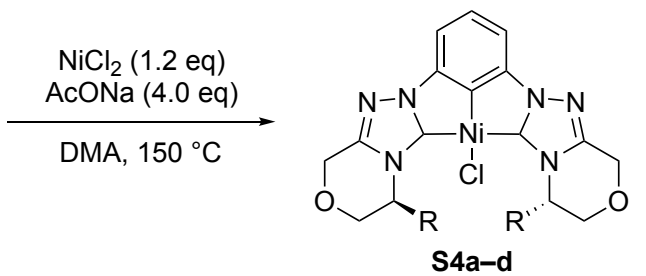

a: $R=P h$

b: $\mathrm{R}={ }^{i} \mathrm{Pr}$

c: $R={ }^{t} \mathrm{Bu}$

S1a-d

S4a-d

The mixture of $\mathbf{S 1}$, anhydrous $\mathrm{NiCl}_{2}$ (1.1 equiv), and $\mathrm{AcONa}$ (4.0 equiv) in $\mathrm{N}, \mathrm{N}$-dimethylacetamide ( $0.1 \mathrm{M}$ in total) was stirred at $150{ }^{\circ} \mathrm{C}$ overnight, and was diluted with $\mathrm{CH}_{2} \mathrm{Cl}_{2}$ after cooling down to room temperature. The mixture was washed with $\mathrm{H}_{2} \mathrm{O}$ for three times, dried over $\mathrm{Na}_{2} \mathrm{SO}_{4}$, and concentrated in vacuo. The residue was purified by silica gel column chromatography ( $n$-hexane/ $\mathrm{CHCl}_{3}$ ) to afford $\mathrm{Ni}(\mathrm{II})$ complex $\mathbf{S 4}$.

Ni(II) pincer complex (S4a): Prepared from S1a $(82.0 \mathrm{mg}, 0.149 \mathrm{mmol})$ and isolated as a yellow solid $(26.3 \mathrm{mg}$, $31 \%)$.

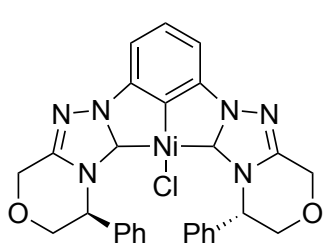
m.p. $>280^{\circ} \mathrm{C}$; IR $\left(\mathrm{CHCl}_{3}\right) 1597,1557,1394,1354,1113,1090,1113,1090 \mathrm{~cm}^{-1}$; ${ }^{1} \mathbf{H}$ NMR $(400 \mathrm{MHz}$, $\left.\mathrm{CDCl}_{3}\right) \delta 7.35-7.23(\mathrm{~m}, 7 \mathrm{H}), 7.17(\mathrm{t}, J=7.0 \mathrm{~Hz}, 1 \mathrm{H}), 7.09-7.03(\mathrm{~m}, 5 \mathrm{H}), 6.37(\mathrm{~d}, J=2.6 \mathrm{~Hz}, 2 \mathrm{H}), 5.21$ $(\mathrm{d}, J=16.1 \mathrm{~Hz}, 2 \mathrm{H}), 4.88(\mathrm{~d}, J=16.1 \mathrm{~Hz}, 2 \mathrm{H}), 4.17(\mathrm{~d}, J=12.2 \mathrm{~Hz}, 2 \mathrm{H}), 4.06(\mathrm{dd}, J=12.2,2.6 \mathrm{~Hz}$, 2H); ${ }^{13} \mathrm{C} \mathrm{NMR}\left(100 \mathrm{MHz}, \mathrm{CD}_{2} \mathrm{Cl}_{2}\right) \delta 175.4,148.6,146.3,143.1,140.3,128.6,128.2,127.0,126.2,109.2$, 70.8, 63.2, 58.2; HRMS (ESI) $m / z$ calc'd for $\mathrm{C}_{28} \mathrm{H}_{23} \mathrm{~N}_{6} \mathrm{O}_{2} \mathrm{Ni}[\mathrm{M}-\mathrm{Cl}]^{+}: 533.1230$, found: 533.1231; $[\alpha]_{D^{22}} 351.9\left(c 0.18, \mathrm{CHCl}_{3}\right)$. 
Ni(II) pincer complex (S4b): Prepared from S1b (340 mg, $0.706 \mathrm{mmol}$ ) and isolated as a yellow solid (253 mg, 71\%). m.p.

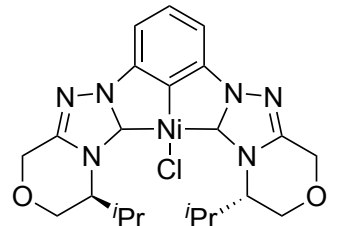

$\left.0.10, \mathrm{CHCl}_{3}\right)$. $>280{ }^{\circ} \mathrm{C}$; IR $\left(\mathrm{CHCl}_{3}\right)$ 1600, 1556, 1488, 1464, 1403, 1361, 1329, 1260, 1144, 1119, $1089 \mathrm{~cm}^{-1}$; ${ }^{1} \mathrm{H}$ NMR $\left(400 \mathrm{MHz}, \mathrm{CDCl}_{3}\right) \delta 7.16(\mathrm{dd}, J=8.3,7.2 \mathrm{~Hz}, 1 \mathrm{H}), 7.040(\mathrm{~d}, J=7.2 \mathrm{~Hz}, 1 \mathrm{H}), 7.039(\mathrm{~d}, J=8.3 \mathrm{~Hz}, 1 \mathrm{H})$, $5.08(\mathrm{~d}, J=15.9 \mathrm{~Hz}, 2 \mathrm{H}), 4.93(\mathrm{dd}, J=4.2,4.2 \mathrm{~Hz}, 2 \mathrm{H}), 4.78(\mathrm{~d}, J=15.9 \mathrm{~Hz}, 2 \mathrm{H}), 4.34(\mathrm{~d}, J=12.7 \mathrm{~Hz}$, $2 \mathrm{H}), 3.81(\mathrm{dd}, J=12.7,2.9 \mathrm{~Hz}, 2 \mathrm{H}), 2.85-2.73(\mathrm{~m}, 2 \mathrm{H}), 1.16(\mathrm{~d}, J=6.9 \mathrm{~Hz}, 6 \mathrm{H}), 0.90(\mathrm{~d}, J=7.1 \mathrm{~Hz}$, $6 \mathrm{H}) ;{ }^{13} \mathrm{C} \mathrm{NMR}\left(100 \mathrm{MHz}, \mathrm{CDCl}_{3}\right) \delta 173.8,147.9,145.7,141.5,125.8,108.7,64.0,61.9,57.8,33.3,19.4$, 17.1; HRMS (ESI) $m / z$ calc'd for $\mathrm{C}_{22} \mathrm{H}_{27} \mathrm{~N}_{6} \mathrm{O}_{2} \mathrm{Ni}[\mathrm{M}-\mathrm{Cl}]^{+}: 465.1543$, found: 465.1543; [ $\left.\alpha\right]_{D^{23}} 163.7$ (c

Ni(II) pincer complex (S4c): Prepared from S1c (495 mg, $2.89 \mathrm{mmol})$ and isolated as a yellow solid (401 mg, 55\%). m.p.<smiles>CC(C)C1COCC2=NN3C4=NN5C(N(Cl)c6c(cccc63)[C@@H](Br)COC4)N2N15</smiles>
$>280{ }^{\circ} \mathrm{C}$; IR $\left(\mathrm{CHCl}_{3}\right)$ 1600, 1553, 1479, 1401, 1369, 1352, 1292, 1127, 1062, $1003 \mathrm{~cm}^{-1}$; ${ }^{1} \mathbf{H}$ NMR $(400$ $\left.\mathrm{MHz}_{2} \mathrm{CDCl}_{3}\right) \delta 7.17(\mathrm{dd}, J=8.3,7.3 \mathrm{~Hz}, 1 \mathrm{H}), 7.031(\mathrm{~d}, J=7.3 \mathrm{~Hz}, 1 \mathrm{H}), 7.030(\mathrm{~d}, J=8.2 \mathrm{~Hz}, 1 \mathrm{H}), 5.16$ $(\mathrm{dd}, J=2.8,1.0 \mathrm{~Hz}, 2 \mathrm{H}), 5.10(\mathrm{~d}, J=16.4 \mathrm{~Hz}, 2 \mathrm{H}), 4.86(\mathrm{~d}, J=16.4 \mathrm{~Hz}, 2 \mathrm{H}), 4.40(\mathrm{dd}, J=12.7,1.0 \mathrm{~Hz}$, 2H), $3.91(\mathrm{dd}, J=12.7,2.8 \mathrm{~Hz}, 2 \mathrm{H}), 1.16$ (s, 18H); ${ }^{13} \mathrm{C}$ NMR $\left(100 \mathrm{MHz}, \mathrm{CDCl}_{3}\right) \delta$ 174.1, 148.5, 145.7, 140.8, 125.8, 108.8, 65.1, 61.2, 59.7, 36.1, 27.9; HRMS (ESI) $m / z$ calc'd for $\mathrm{C}_{24} \mathrm{H}_{31} \mathrm{~N}_{6} \mathrm{O}_{2} \mathrm{Ni}[\mathrm{M}-\mathrm{Cl}]^{+}$:

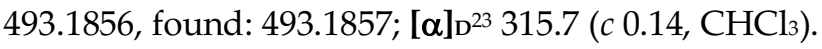

Ni(II) pincer complex (S4d): Prepared from S1d (400 mg, $0.693 \mathrm{mmol})$ and isolated as a yellow solid (294 mg, $71 \%)$. m.p.<smiles>ClN1c2ccccc2CN2c3cccc4c3N(C12)N1C(=N4)COCC1Br</smiles>
157-159 ${ }^{\circ} \mathrm{C}$; IR $\left(\mathrm{CHCl}_{3}\right)$ 1601, 1557, 1357, 1123, 1090, $1064 \mathrm{~cm}^{-1} ; \mathbf{1}^{\mathbf{H}} \mathbf{N M R}\left(400 \mathrm{MHz}, \mathrm{CDCl}_{3}\right) \delta 7.66-$ $7.60(\mathrm{~m}, 4 \mathrm{H}), 7.40-7.34(\mathrm{~m}, 4 \mathrm{H}), 7.32-7.24(\mathrm{~m}, 2 \mathrm{H}), 7.18(\mathrm{dd}, J=8.4,7.0 \mathrm{~Hz}, 1 \mathrm{H}), 7.09-7.04(\mathrm{~m}, 2 \mathrm{H})$, $5.37(\mathrm{ddd}, J=12.6,4.2,2.7 \mathrm{~Hz}, 2 \mathrm{H}), 5.17(\mathrm{~d}, J=15.9 \mathrm{~Hz}, 2 \mathrm{H}), 4.85(\mathrm{~d}, J=15.9 \mathrm{~Hz}, 2 \mathrm{H}), 4.03(\mathrm{~d}, J=$ $12.6 \mathrm{~Hz}, 2 \mathrm{H}), 3.85(\mathrm{dd}, J=12.6,4.2 \mathrm{~Hz}, 2 \mathrm{H}), 3.68(\mathrm{ddd}, J=12.6,2.8,1.3 \mathrm{~Hz}, 2 \mathrm{H}), 3.07(\mathrm{dd}, J=12.6$, $12.6 \mathrm{~Hz}, 2 \mathrm{H}) ;{ }^{13} \mathrm{C}$ NMR $\left(100 \mathrm{MHz}, \mathrm{CDCl}_{3}\right) \delta 173.8,147.3,145.7,141.8,136.7,130.0,128.6,126.9$, 125.8, 108.8, 64.7, 62.7, 55.6, 39.8; HRMS (ESI) $m / z$ calc'd for $\mathrm{C}_{30} \mathrm{H}_{27} \mathrm{~N}_{6} \mathrm{O}_{2} \mathrm{Ni}[\mathrm{M}-\mathrm{Cl}]^{+:}$561.1543, found: $561.1548 ;[\alpha]_{D^{24}}-30.6\left(c 0.10, \mathrm{CHCl}_{3}\right)$.

\section{Cationic Ni(II) pincer complex (Ni/L2):}
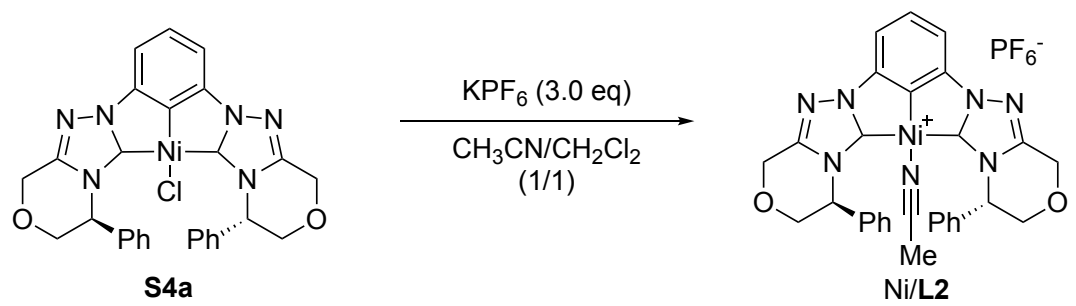

The mixture of S4a (228 mg, $0.400 \mathrm{mmol})$ and $\mathrm{KPF}_{6}(221 \mathrm{mg}, 1.20 \mathrm{mmol})$ in $\mathrm{CH}_{3} \mathrm{CN} / \mathrm{CH}_{2} \mathrm{Cl}_{2}(1 / 1,10 \mathrm{~mL})$ was stirred for 1 $\mathrm{h}$ at room temperature. After filtration through Celite, the volatiles were removed in vacuo. The residue was dissolved in minimum amount of $\mathrm{CH}_{2} \mathrm{Cl}_{2}$, and solidified by the addition of $\mathrm{Et}_{2} \mathrm{O}$ to afford $\mathrm{Ni} / \mathrm{L2}(269 \mathrm{mg}, 93 \%)$.

m.p. 262-264 ${ }^{\circ} \mathrm{C}$; IR $\left(\mathrm{CHCl}_{3}\right)$ 2285, 1604, 1558, 1497, 1455, 1402, 1361, 1119, $1095 \mathrm{~cm}^{-1}$; $1 \mathbf{H}$ NMR $\left(400 \mathrm{MHz}, \mathrm{CD}_{2} \mathrm{Cl} 2\right) \delta 7.52-$ $7.36(\mathrm{~m}, 6 \mathrm{H}), 7.28(\mathrm{t}, J=7.6 \mathrm{~Hz}, 1 \mathrm{H}), 7.14(\mathrm{~d}, J=7.6 \mathrm{~Hz}, 2 \mathrm{H}), 6.99(\mathrm{~d}, J=6.5 \mathrm{~Hz}, 4 \mathrm{H}), 5.47(\mathrm{~s}, 2 \mathrm{H}), 5.14(\mathrm{~d}, J=16.4 \mathrm{~Hz}, 2 \mathrm{H})$, $4.91(\mathrm{~d}, J=16.4 \mathrm{~Hz}, 2 \mathrm{H}), 4.32(\mathrm{~d}, J=12.5 \mathrm{~Hz}, 2 \mathrm{H}), 4.20(\mathrm{dd}, J=12.5,3.2 \mathrm{~Hz}, 2 \mathrm{H}), 1.85(\mathrm{~s}, 3 \mathrm{H}) ;{ }^{13} \mathrm{C} \mathrm{NMR}(100 \mathrm{MHz}, \mathrm{CD} 2 \mathrm{Cl} 2)$ $\delta 175.5,149.5,146.4,140.0,138.5,129.8,129.2,128.6,127.9,125.9,110.5,71.3,63.4,58.3,3.8 ;{ }^{19} \mathrm{~F} \mathrm{NMR}\left(376 \mathrm{MHz}, \mathrm{CD}_{2} \mathrm{Cl}_{2}\right) \delta$ $-72.7(\mathrm{~d}, J=710 \mathrm{~Hz})$; ${ }^{31 P} \mathrm{NMR}\left(162 \mathrm{MHz}, \mathrm{CD}_{2} \mathrm{Cl}_{2}\right) \delta-144.5$ (sept, $\left.J=710 \mathrm{~Hz}\right)$; HRMS (ESI) $m / z$ calc'd for $\mathrm{C}_{30} \mathrm{H}_{26} \mathrm{~N}_{7} \mathrm{O}_{2}[\mathrm{M}-$ $\left.\mathrm{PF}_{6}\right]^{+}$: 574.1496, found: 574.1498; [ $\left.\alpha\right]_{\mathrm{D}^{23}} 367.2$ (c 0.10, $\left.\mathrm{CHCl}_{3}\right)$. 


\section{Catalytic Asymmetric Addition to Imines}

\section{3-1. Brief ligand screening}
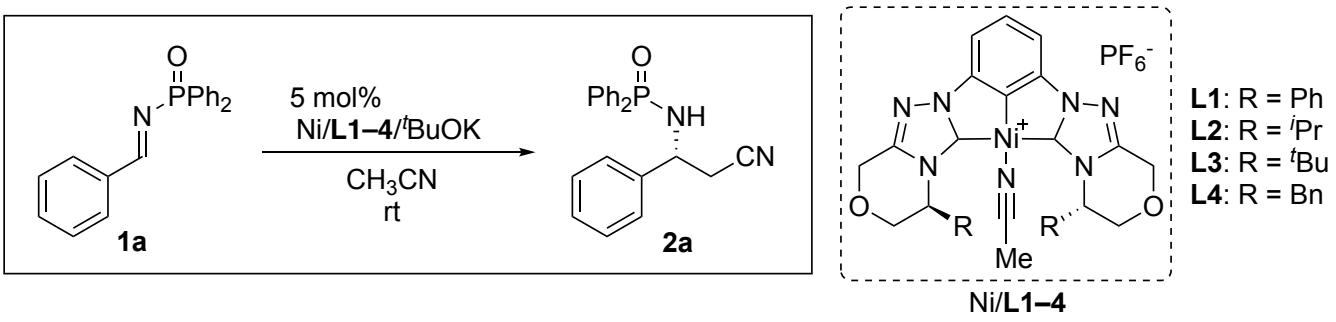

To a suspension of $\mathrm{AgPF}_{6}(25.3 \mathrm{mg}, 0.010 \mathrm{mmol})$ in $\mathrm{CH}_{2} \mathrm{Cl}_{2}(1.0 \mathrm{M})$ was added $\mathrm{Ni}(\mathrm{II})$ complex $\mathrm{S} 4$ (0.010 mmol). The resulting mixture was stirred for $1 \mathrm{~h}$ at room temperature, and the white precipitate was removed by filtration through Celite. After removal of solvent by evaporation, To the residue in a $20 \mathrm{~mL}$ test tube were successively added ${ }^{\mathrm{B}} \mathrm{BuOK}(1.1$ $\mathrm{mg}, 0.010 \mathrm{mmol})$ and $\mathrm{CH}_{3} \mathrm{CN}(1.0 \mathrm{~mL})$ at $0{ }^{\circ} \mathrm{C}$. The solution was stirred for $10 \mathrm{~min}$ at the same temperature and regarded as $0.01 \mathrm{M}$ catalyst solution. To a solution of $1 \mathrm{a}(30.5 \mathrm{mg}, 0.100 \mathrm{mmol})$ in $\mathrm{CH}_{3} \mathrm{CN}(500 \mu \mathrm{L})$ was added the aforementioned catalyst solution ( $500 \mu \mathrm{L}, 0.005 \mathrm{mmol}, 5 \mathrm{~mol} \%$ ) at room temperature. After the complete consumption of $1 \mathrm{a}$ was checked by TLC, the reaction was quenched with $0.1 \mathrm{M} \mathrm{AcOH} / \mathrm{CH}_{3} \mathrm{CN}$ solution $(100 \mu \mathrm{L})$. After the addition of sat. $\mathrm{NaHCO}_{3}$ aqueous solution, the mixture was extracted with AcOEt, and the combined organic layers were washed with brine, dried over $\mathrm{Na}_{2} \mathrm{SO}_{4}$, and concentrated in vacuo. The yield of $2 \mathrm{a}$ was determined by ${ }^{1} \mathrm{H}$ NMR using $1,3,5-$ trimethoxybenzene as an internal standard.

\section{3-2. General procedure for direct catalytic asymmetric addition to imines}
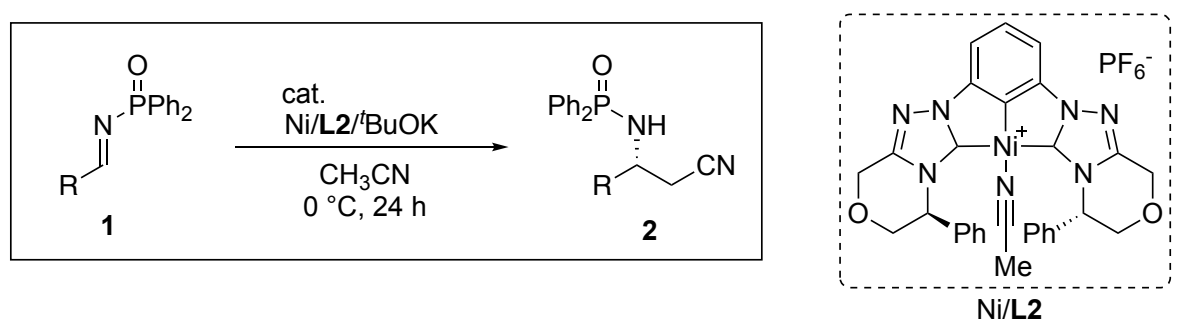

A flame-dried test tube $(20 \mathrm{~mL})$ was charged with $\mathrm{Ni} / \mathrm{L} 2(7.2 \mathrm{mg}, 0.010 \mathrm{mmol})$ and ${ }^{~} \mathrm{BuOK}(1.1 \mathrm{mg}, 0.010 \mathrm{mmol})$ in a dry box. After the test tube was cooled down to $0{ }^{\circ} \mathrm{C}, \mathrm{CH}_{3} \mathrm{CN}(1.0 \mathrm{~mL})$ was added, and the resulting solution was stirred for $10 \mathrm{~min}$ to afford $0.01 \mathrm{M} \mathrm{Ni}$ (II) catalyst solution. The catalyst solution was quickly used within $1 \mathrm{~h}$ after preparation. A solution of $1(0.100 \mathrm{mmol})$ in $\mathrm{CH}_{3} \mathrm{CN}\left(800 \mu \mathrm{L}\right.$ or $500 \mu \mathrm{L}$ ) was cooled to $0{ }^{\circ} \mathrm{C}$, and $200 \mu \mathrm{L}$ (for $2 \mathrm{~mol} \%$ ) or $500 \mu \mathrm{L}$ (for 5 $\mathrm{mol} \%$ ) of the $\mathrm{Ni}(\mathrm{II})$ catalyst solution was slowly added. The final concentration of 2 in $\mathrm{CH}_{3} \mathrm{CN}$ was $0.1 \mathrm{M}$. The resulting mixture was stirred for $24 \mathrm{~h}$ at $0{ }^{\circ} \mathrm{C}$, and quenched with $0.1 \mathrm{M} \mathrm{AcOH} / \mathrm{CH}_{3} \mathrm{CN}$ solution $(100 \mu \mathrm{L})$. After the addition of sat. $\mathrm{NaHCO}_{3}$ aqueous solution, the mixture was extracted with AcOEt, and the combined organic layers were washed with brine, dried over $\mathrm{Na}_{2} \mathrm{SO}_{4}$, and concentrated in vacuo. The residue was purified with silica gel column chromatography (n-hexane/AcOEt) to afford pure $\alpha$-aminonitrile 2. 
3-3. Characterization of Mannich adducts

(R)-N-(2-Cyano-1-phenylethyl)-P,P-diphenylphosphinic amide (2a): Prepared by the general procedure from 1a (30.5<smiles>N#CCC(NP(=O)(O)O)c1ccccc1</smiles>
$\mathrm{mg}, 0.100 \mathrm{mmol})$ and $2 \mathrm{~mol} \%$ catalyst, and isolated as a white solid $(33.0 \mathrm{mg}, 97 \%)$. m.p. $146-148^{\circ} \mathrm{C}$; IR ( $\left.\mathrm{CHCl}_{3}\right): 3148,2893,2251,1591,1456,1439,1186,1124,1110,1071,954 \mathrm{~cm}^{-1} ; \mathbf{H}$ NMR $\left(400 \mathrm{MHz}, \mathrm{CDCl}_{3}\right) \delta$ 7.90-7.29 (m, 4H), 7.56-7.30 (m, 11H), 4.59-4.51 (m, 1H), $3.80(\mathrm{dd}, J=10.7,6.9 \mathrm{~Hz}, 1 \mathrm{H}), 3.06(\mathrm{dd}, J=16.6$, $7.1 \mathrm{~Hz}, 1 \mathrm{H}), 2.95(\mathrm{dd}, J=16.6,4.4 \mathrm{~Hz}, 1 \mathrm{H}) ;{ }^{13} \mathrm{C}$ NMR $\left(100 \mathrm{MHz}, \mathrm{CDCl}_{3}\right) \delta 139.9(\mathrm{~d}, J=6.6 \mathrm{~Hz}), 132.4(\mathrm{~d}, J$ $=9.8 \mathrm{~Hz}), 132.31(\mathrm{~d}, J=2.8 \mathrm{~Hz}), 132.26(\mathrm{~d}, J=2.8 \mathrm{~Hz}), 132.22(\mathrm{~d}, J=76.0 \mathrm{~Hz}), 131.7(\mathrm{~d}, J=9.8 \mathrm{~Hz}), 130.9$ $(\mathrm{d}, J=78.4 \mathrm{~Hz}), 129.1,128.70(\mathrm{~d}, J=12.7 \mathrm{~Hz}), 128.69(\mathrm{~d}, J=12.7 \mathrm{~Hz}), 128.5,126.0,117.2,51.5,28.2(\mathrm{~d}, J=$ $2.7 \mathrm{~Hz}) ;{ }^{31} \mathbf{P}$ NMR $\left(162 \mathrm{MHz}, \mathrm{CDCl}_{3}\right) \delta 23.7$; HRMS (ESI) $\mathrm{m} / z$ calc'd for $\mathrm{C}_{21} \mathrm{H}_{20} \mathrm{~N}_{2} \mathrm{OP}[\mathrm{M}+\mathrm{H}]^{+}$: 347.1308, found: 347.1311; $[\alpha]_{D^{24}} 22.53$ (c 0.65, $\mathrm{CHCl}_{3}, 97 \%$ ee sample); HPLC (CHIRALCEL IC-3 $(\phi 0.46 \mathrm{~cm} \times 25 \mathrm{~cm}), n$-hexane/2-propanol $=2 / 1$, flow rate $1.0 \mathrm{~mL} / \mathrm{min}$, detection at $254 \mathrm{~nm}, \mathrm{tr}_{\mathrm{R}}=11.8 \mathrm{~min}$ (minor), $15.7 \mathrm{~min}$ (major).

Racemic sample

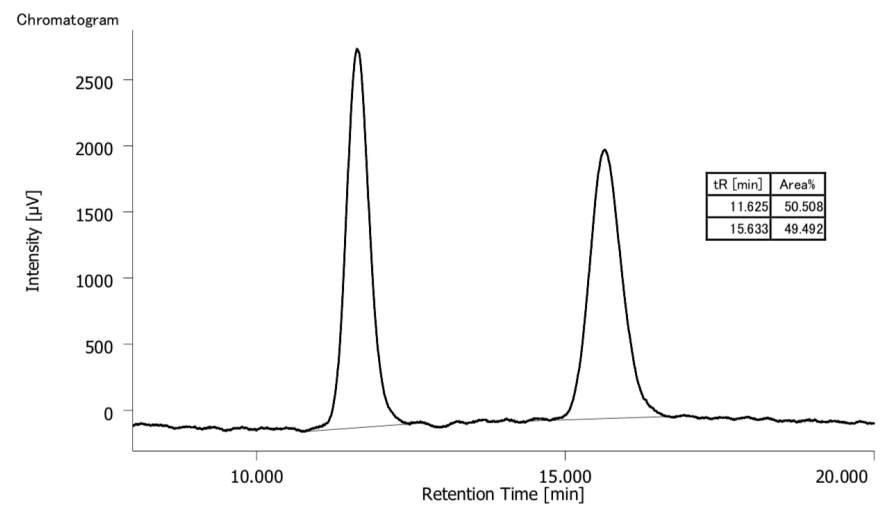

Reaction sample

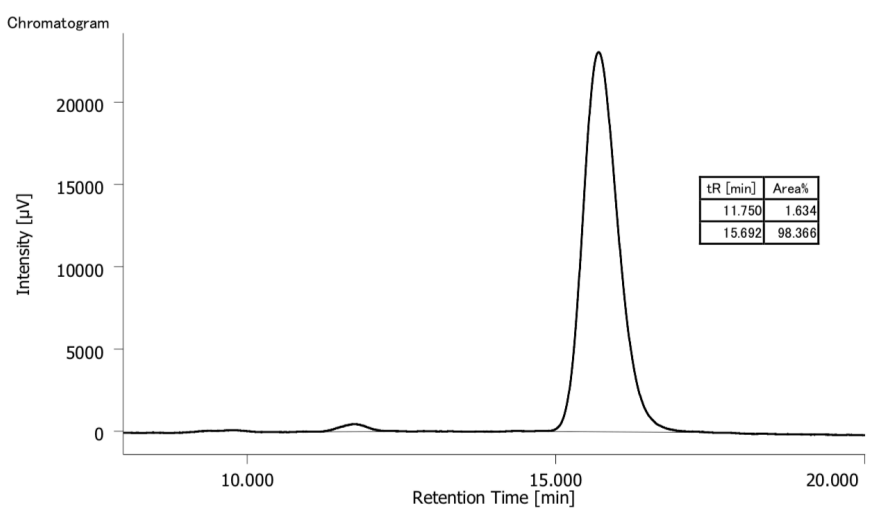

(R)-N-(2-Cyano-1-(o-tolyl)ethyl)-P,P-diphenylphosphinic amide (2b): Prepared by the general procedure from $\mathbf{1 b}$ (31.9<smiles>N#CCC(NP=O)c1ccccc1N</smiles>
$\mathrm{mg}, 0.100 \mathrm{mmol})$ and $5 \mathrm{~mol} \%$ catalyst, and isolated as a white solid $(32.7 \mathrm{mg}, 91 \%) . \mathbf{m . p .} \cdot 213-215^{\circ} \mathrm{C}$; IR (CHCl $): 3136,2899,2252,1590,1474,1464,1438,1194,1171,1119,1082,960 \mathrm{~cm}^{-1} ;{ }^{1} \mathbf{H}$ NMR $(400 \mathrm{MHz}$, $\left.\mathrm{CDCl}_{3}\right) \delta$ 7.90-7.75 (m, 4H), 7.57-7.37 (m, 7H), 7.37-7.21 (m, 2H), $7.14(\mathrm{~d}, J=7.5 \mathrm{~Hz}, 1 \mathrm{H}), 4.86-4.75(\mathrm{~m}$, $1 \mathrm{H}), 3.59(\mathrm{dd}, J=9.9,7.9 \mathrm{~Hz}, 1 \mathrm{H}), 3.04(\mathrm{dd}, J=16.5,7.3 \mathrm{~Hz}, 1 \mathrm{H}), 2.90(\mathrm{dd}, J=16.5,4.2 \mathrm{~Hz}, 1 \mathrm{H}), 2.07(\mathrm{~s}$, $3 \mathrm{H}) ;{ }^{13} \mathrm{C}$ NMR $\left(100 \mathrm{MHz}, \mathrm{CDCl}_{3}\right) \delta 138.5(\mathrm{~d}, J=6.4 \mathrm{~Hz}), 134.5,132.4(\mathrm{~d}, J=9.8 \mathrm{~Hz}), 132.4(\mathrm{~d}, J=2.8 \mathrm{~Hz})$, $132.3(\mathrm{~d}, J=2.8 \mathrm{~Hz}), 132.2(\mathrm{~d}, J=76.2 \mathrm{~Hz}), 130.9,130.9(\mathrm{~d}, J=78.9 \mathrm{~Hz}), 128.72(\mathrm{~d}, J=12.7 \mathrm{~Hz}), 128.66(\mathrm{~d}, J$ $=12.7 \mathrm{~Hz}), 128.3,127.0,124.9,117.0,47.3,27.9(\mathrm{~d}, J=2.5 \mathrm{~Hz}), 19.0 ;{ }^{31 P}$ NMR $\left(162 \mathrm{MHz}, \mathrm{CDCl}_{3}\right) \delta 23.8 ; \mathrm{HRMS}(\mathrm{ESI}) \mathrm{m} / \mathrm{z}$ calc'd for $\mathrm{C}_{22} \mathrm{H}_{22} \mathrm{ON}_{2} \mathrm{P}[\mathrm{M}+\mathrm{H}]^{+}: 361.1464$, found: 361.1464; $[\alpha]_{\mathrm{D}^{24}}-35.5$ (c $0.10, \mathrm{CHCl}_{3}, 96 \%$ ee sample); HPLC $\left(\right.$ CHIRALCEL IC-3 $(\phi 0.46 \mathrm{~cm} \times 25 \mathrm{~cm}), n$-hexane $/ 2$-propanol $=2 / 1$, flow rate $1.0 \mathrm{~mL} / \mathrm{min}$, detection at $254 \mathrm{~nm}, \mathrm{t}_{\mathrm{R}}=9.5 \mathrm{~min}$ (minor), $16.2 \mathrm{~min}$ (major).

Racemic sample

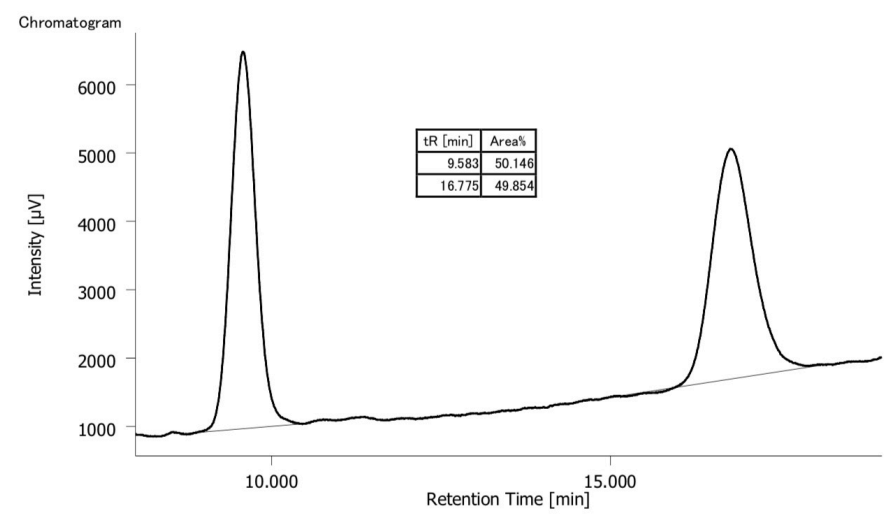

Reaction sample

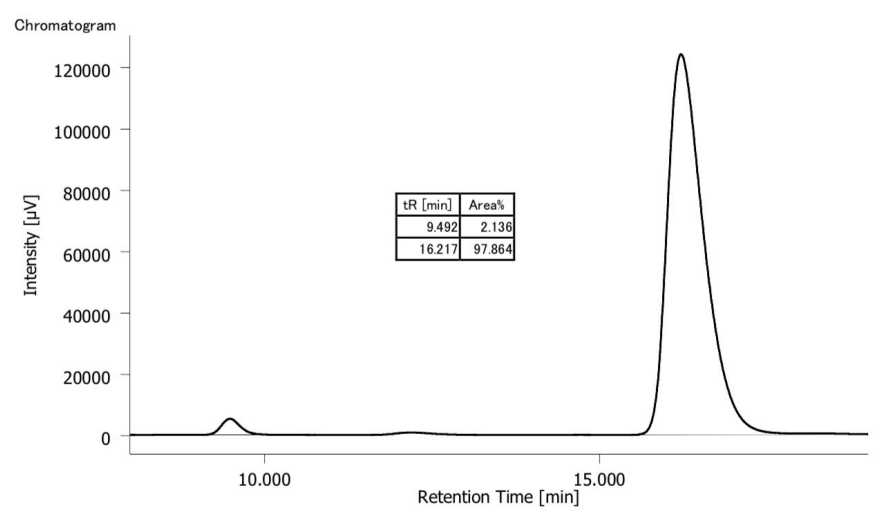


(R)-N-(2-Cyano-1-(naphthalen-2-yl)ethyl)-P,P-diphenylphosphinic amide (2c): Prepared by the general procedure from $1 \mathrm{c}(35.5 \mathrm{mg}, 0.100 \mathrm{mmol}), 2 \mathrm{~mol} \%$ catalyst, and THF $(100 \mu \mathrm{L})$ as a cosolvent, and isolated as a white solid $(39.1 \mathrm{mg}$,

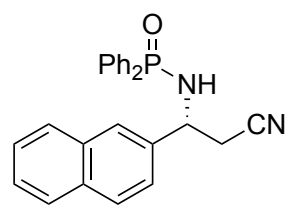
99\%). m.p. 165-168 ${ }^{\circ} \mathrm{C}$; IR $\left(\mathrm{CHCl}_{3}\right): 3123,2898,2250,1592,1482,1456,1439,1188,1178,1125,1098$ $\mathrm{cm}^{-1}$; ${ }^{1} \mathrm{H}$ NMR $\left(400 \mathrm{MHz}, \mathrm{CDCl}_{3}\right) \delta$ 7.94-7.82 (m, 7H), $7.78(\mathrm{~s}, 1 \mathrm{H}), 7.58-7.39(\mathrm{~m}, 9 \mathrm{H}), 4.81-4.71(\mathrm{~m}$, $1 \mathrm{H}), 3.68(\mathrm{dd}, J=10.7,7.1 \mathrm{~Hz}, 1 \mathrm{H}), 3.21(\mathrm{dd}, J=16.6,6.9 \mathrm{~Hz}, 1 \mathrm{H}), 3.06(\mathrm{dd}, J=16.6,4.1 \mathrm{~Hz}, 1 \mathrm{H}) ;{ }^{13} \mathrm{C}$ NMR $\left(100 \mathrm{MHz}, \mathrm{CDCl}_{3}\right) \delta 137.2(\mathrm{~d}, J=6.7 \mathrm{~Hz}), 133.1(\mathrm{~d}, J=6.8 \mathrm{~Hz}), 132.38(\mathrm{~d}, J=9.7 \mathrm{~Hz}), 132.34(\mathrm{~d}$, $J=2.7 \mathrm{~Hz}), 132.28(\mathrm{~d}, J=2.5 \mathrm{~Hz}), 132.24(\mathrm{~d}, J=76.0 \mathrm{~Hz}), 131.7(\mathrm{~d}, J=9.8 \mathrm{~Hz}), 131.0(\mathrm{~d}, J=78.1 \mathrm{~Hz})$, 129.2, $128.7(\mathrm{~d}, J=12.7 \mathrm{~Hz}), 128.1,127.7,126.6,126.5,125.1,123.7,117.2,51.6,28.2(\mathrm{~d}, J=2.0 \mathrm{~Hz}) ;{ }^{31 P} \mathrm{NMR}(162 \mathrm{MHz}$, $\left.\mathrm{CDCl}_{3}\right) \delta$ 23.9; HRMS (ESI) $m / z$ calc'd for $\mathrm{C}_{25} \mathrm{H}_{22} \mathrm{ON}_{2} \mathrm{P}[\mathrm{M}+\mathrm{H}]^{+}: 397.1464$, found: $397.1471 ;[\alpha]_{\mathrm{D}^{24}} 56.7$ (c $0.10, \mathrm{CHCl}_{3}, 98 \%$ ee sample); HPLC (CHIRALCEL IC-3 $(\phi 0.46 \mathrm{~cm} \times 25 \mathrm{~cm}), n$-hexane $/ 2$-propanol = 2/1, flow rate $1.0 \mathrm{~mL} / \mathrm{min}$, detection at $254 \mathrm{~nm}, \mathrm{t}_{\mathrm{R}}=14.5 \mathrm{~min}$ (minor), $20.6 \mathrm{~min}$ (major).

Racemic sample

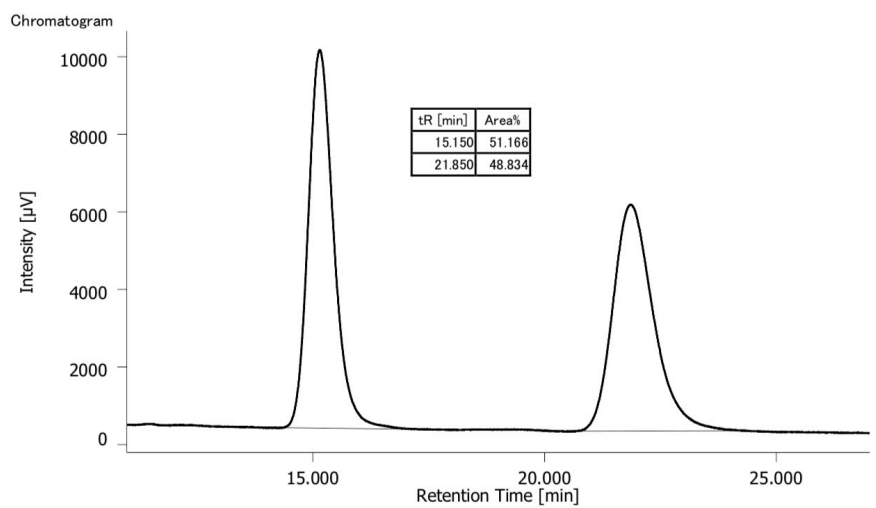

Reaction sample

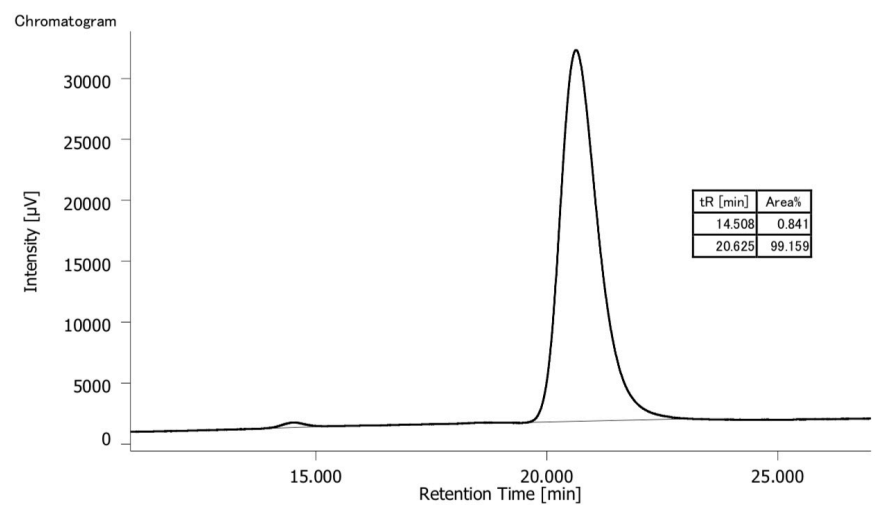

(R)-N-(2-Cyano-1-(naphthalen-1-yl)ethyl)-P,P-diphenylphosphinic amide (2d): Prepared by the general procedure<smiles>N#CCC(N[P+](=O)[O-])c1cccc2ccccc12</smiles>
from $1 \mathrm{~d}(35.5 \mathrm{mg}, 0.100 \mathrm{mmol})$ and $2 \mathrm{~mol} \%$ catalyst, and isolated as a white solid $(38.5 \mathrm{mg}, 97 \%)$. m.p. 171-173 ${ }^{\circ} \mathrm{C}$; IR ( $\left.\mathrm{CHCl}_{3}\right): 3168,2901,2250,1593,1514,1438,1413,1175,1124,1109,1075 \mathrm{~cm}^{-1}$; ${ }^{1} \mathbf{H}$ NMR $\left(400 \mathrm{MHz}, \mathrm{CDCl}_{3}\right) \delta$ 7.92-7.78 (m, 6H), $7.74(\mathrm{~d}, J=8.4 \mathrm{~Hz}, 2 \mathrm{H}), 7.71(\mathrm{~d}, J=7.1 \mathrm{~Hz}, 2 \mathrm{H}), 7.58-7.43(\mathrm{~m}$, $7 \mathrm{H}), 7.39-7.34(\mathrm{~m}, 2 \mathrm{H}), 5.50-5.40(\mathrm{~m}, 1 \mathrm{H}), 3.77(\mathrm{dd}, J=10.4,7.5 \mathrm{~Hz}, 1 \mathrm{H}), 3.33(\mathrm{dd}, J=16.6,6.6 \mathrm{~Hz}, 1 \mathrm{H})$, $3.09(\mathrm{dd}, J=16.6,4.0 \mathrm{~Hz}, 1 \mathrm{H}) ;{ }^{13} \mathrm{C}$ NMR $\left(100 \mathrm{MHz}, \mathrm{CDCl}_{3}\right) \delta 135.4(\mathrm{~d}, J=6.6 \mathrm{~Hz}), 132.3,132.23(\mathrm{~d}, J=$ $9.9 \mathrm{~Hz}), 132.22(\mathrm{~d}, J=62.5 \mathrm{~Hz}), 130.9(\mathrm{~d}, J=65.0 \mathrm{~Hz}), 131.7(\mathrm{~d}, J=9.8 \mathrm{~Hz}), 130.9(\mathrm{~d}, J=65.0 \mathrm{~Hz}), 129.6$, $129.2(\mathrm{~d}, J=1.7 \mathrm{~Hz}), 128.7(\mathrm{~d}, J=12.7 \mathrm{~Hz}), 128.6(\mathrm{~d}, J=12.7 \mathrm{~Hz}), 126.8,126.0,125.3,123.4,121.9,117.2,47.2,27.9(\mathrm{~d}, J=2.2$ $\mathrm{Hz}$ ); ${ }^{31}$ P NMR (162 MHz, CDCl $) \delta$ 24.0; HRMS (ESI) $m / z$ calc'd for $\mathrm{C}_{25} \mathrm{H}_{22} \mathrm{ON}_{2} \mathrm{P}[\mathrm{M}+\mathrm{H}]^{+}:$397.1464, found: 397.1460; $[\boldsymbol{\alpha}]_{\mathrm{D}^{24}}$ -50.2 ( $0.10, \mathrm{CHCl}_{3}, 95 \%$ ee sample); HPLC (CHIRALCEL IA-3 $(\phi 0.46 \mathrm{~cm} \times 25 \mathrm{~cm}), n$-hexane/2-propanol = 2/1, flow rate $1.0 \mathrm{~mL} / \mathrm{min}$, detection at $254 \mathrm{~nm}, \mathrm{t}_{\mathrm{R}}=11.5 \mathrm{~min}$ (minor), $14.5 \mathrm{~min}$ (major).

Racemic sample

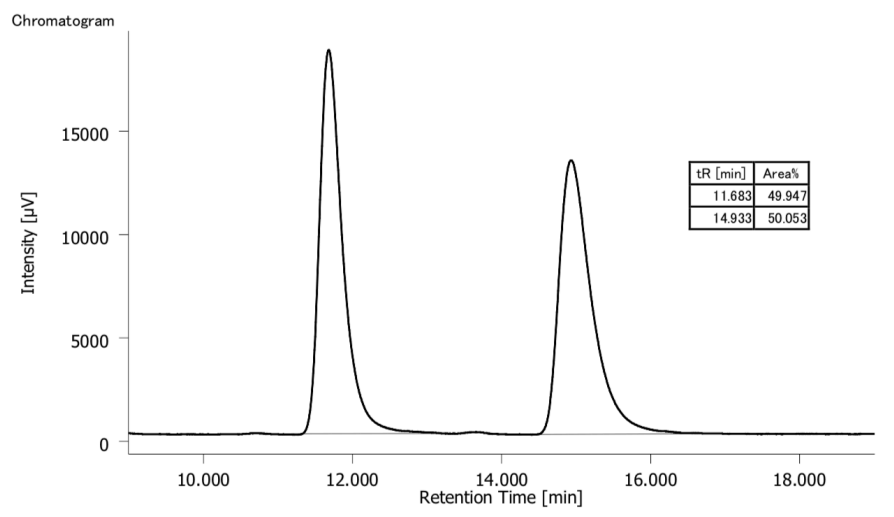

Reaction sample

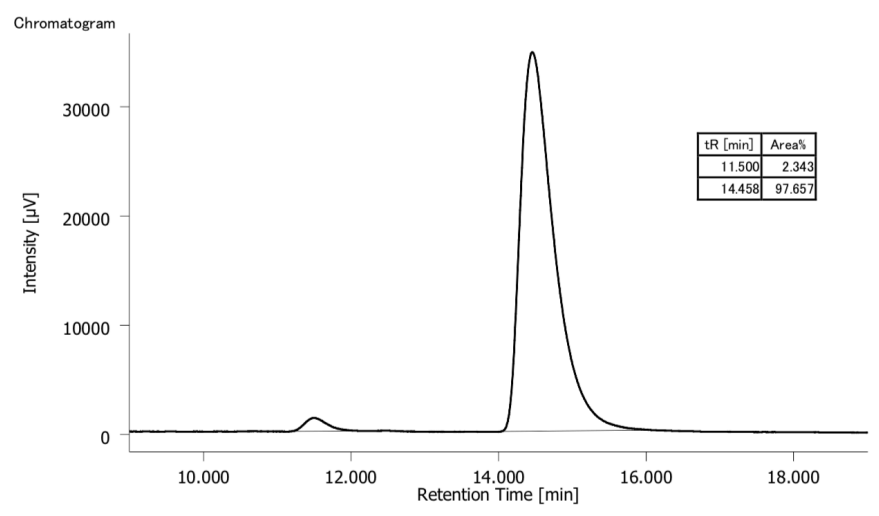


(R)-N-(2-Cyano-1-(4-methoxyphenyl)ethyl)-P,P-diphenylphosphinic amide (2e): Prepared by the general procedure<smiles>COc1ccc(C(CC#N)NP(=O)(O)c2ccccc2)cc1</smiles>
from 1 e $(33.5 \mathrm{mg}, 0.100 \mathrm{mmol})$ and $2 \mathrm{~mol} \%$ catalyst, and isolated as a white solid (36.1 $\mathrm{mg}, 96 \%)$. m.p. $115-117^{\circ} \mathrm{C}$; IR $\left(\mathrm{CHCl}_{3}\right): 3149,2902,2250,1613,1590,1515,1439,1251,1180,1122,1028 \mathrm{~cm}^{-1}$; ${ }_{1} \mathrm{H}$ NMR $\left(400 \mathrm{MHz}, \mathrm{CDCl}_{3}\right) \delta$ 7.91-7.81 (m, 4H), 7.57-7.50 (m, 2H), 7.50-7.41 (m, 4H), 7.29-7.24 (m, $2 \mathrm{H}), 6.93-6.88(\mathrm{~m}, 2 \mathrm{H}), 4.59-4.49(\mathrm{~m}, 1 \mathrm{H}), 3.81(\mathrm{~s}, 3 \mathrm{H}), 3.49(\mathrm{dd}, J=10.4,7.1 \mathrm{~Hz}, 1 \mathrm{H}), 3.06(\mathrm{dd}, J=$ $16.6,6.9 \mathrm{~Hz}, 1 \mathrm{H}), 2.94(\mathrm{dd}, J=16.6,4.2 \mathrm{~Hz}, 1 \mathrm{H}) ;{ }^{13} \mathrm{C}$ NMR $\left(100 \mathrm{MHz}, \mathrm{CDCl}_{3}\right) \delta 159.5,132.32(\mathrm{~d}, J=$ $9.7 \mathrm{~Hz}), 132.31(\mathrm{q}, J=69.8 \mathrm{~Hz}), 132.22(\mathrm{~d}, J=3.3 \mathrm{~Hz}), 132.2(\mathrm{~d}, J=6.6 \mathrm{~Hz}), 132.2,132.1(\mathrm{~d}, J=6.8 \mathrm{~Hz})$, $131.7(\mathrm{~d}, J=9.8 \mathrm{~Hz}), 131.0(\mathrm{~d}, J=72.1 \mathrm{~Hz}), 128.6(\mathrm{q}, J=12.6 \mathrm{~Hz}), 127.3,117.3,114.3,55.3,51.0,28.2(\mathrm{~d}, J=2.9 \mathrm{~Hz}) ;{ }^{31}$ P NMR

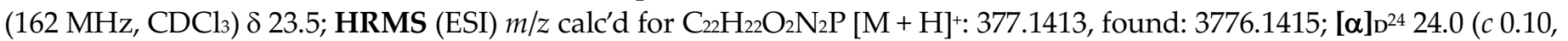
$\mathrm{CHCl}_{3}, 97 \%$ ee sample); HPLC (CHIRALCEL IC-3 $(\phi 0.46 \mathrm{~cm} \times 25 \mathrm{~cm}), n$-hexane/2-propanol = 2/1, flow rate $1.0 \mathrm{~mL} / \mathrm{min}$, detection at $254 \mathrm{~nm}, \mathrm{tr}_{\mathrm{r}}=16.9 \mathrm{~min}$ (minor), $22.4 \mathrm{~min}$ (major).

Racemic sample

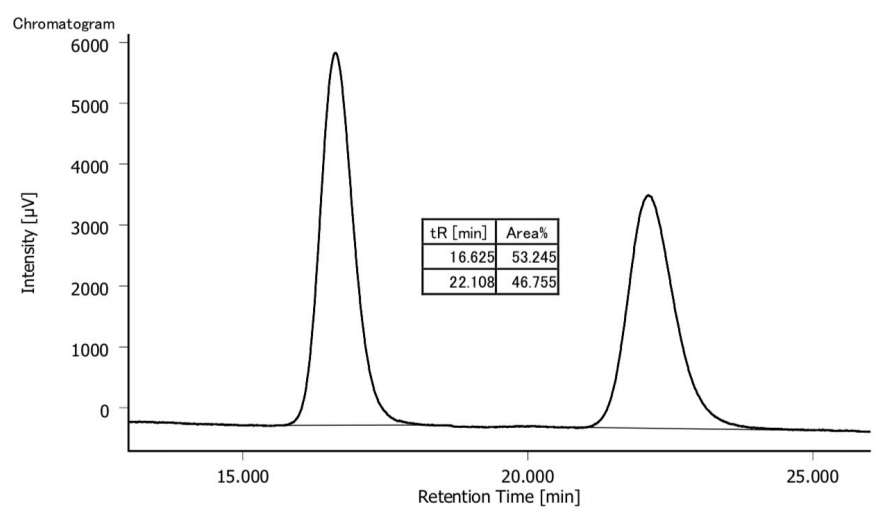

Reaction sample

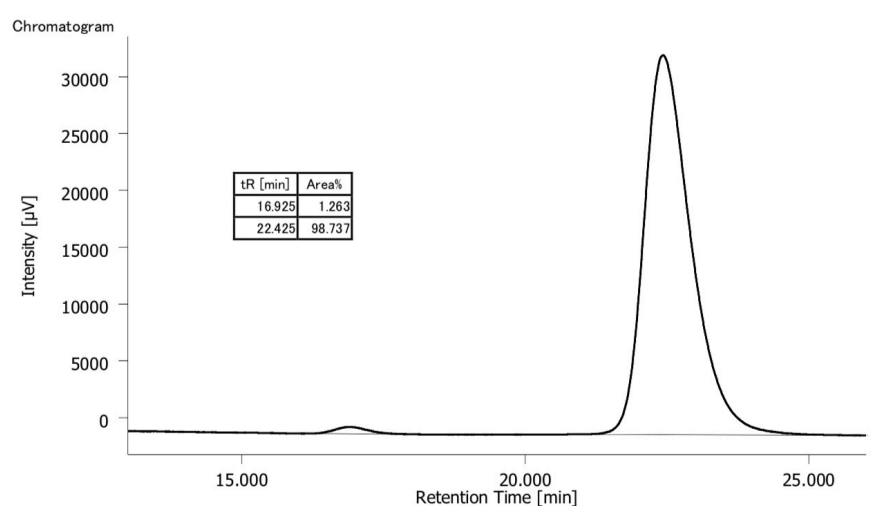

(R)-N-(2-Cyano-1-(4-chlorophenyl)ethyl)-P,P-diphenylphosphinic amide (2f): Prepared by the general procedure from<smiles>N#CCCc1ccc(Cl)cc1NP(=O)=O</smiles>
If (34.0 mg, $0.100 \mathrm{mmol})$ and $2 \mathrm{~mol} \%$ catalyst, and isolated as a white solid $(36.8 \mathrm{mg}, 97 \%)$. m.p. 163-165 ${ }^{\circ} \mathrm{C}$; IR ( $\left.\mathrm{CHCl}_{3}\right): 3153,2895,2251,1592,1493,1439,1181,1124,1110,1092,1014 \mathrm{~cm}^{-1} ; 1 \mathbf{H}$ NMR $\left(400 \mathrm{MHz}, \mathrm{CDCl}_{3}\right) \delta$ 7.92-7.80 (m, 4H), 7.58-7.42 (m, 6H), 7.38-7.33 (m, 2H), 7.31-7.27 (m, 2H), 4.62$4.52(\mathrm{~m}, 1 \mathrm{H}), 3.54(\mathrm{dd}, J=10.7,6.7 \mathrm{~Hz}, 1 \mathrm{H}), 3.08(\mathrm{dd}, J=16.7,6.7 \mathrm{~Hz}, 1 \mathrm{H}), 2.93(\mathrm{dd}, J=16.7,4.3 \mathrm{~Hz}$, 1H); ${ }^{13} \mathrm{C}$ NMR $\left(100 \mathrm{MHz}, \mathrm{CDCl}_{3}\right) \delta 138.5(\mathrm{~d}, J=6.2 \mathrm{~Hz}), 134.3,132.3(\mathrm{~d}, J=2.9 \mathrm{~Hz}), 132.2(\mathrm{~d}, J=9.8$ $\mathrm{Hz}), 131.7(\mathrm{~d}, J=9.8 \mathrm{~Hz}), 130.5(\mathrm{~d}, J=52.8 \mathrm{~Hz}), 129.2,128.8(\mathrm{~d}, J=2.2 \mathrm{~Hz}), 128.6(\mathrm{~d}, J=2.2 \mathrm{~Hz}), 127.5$, 117.0, 51.0, $28.0(\mathrm{~d}, J=3.6 \mathrm{~Hz}) ;{ }^{31}$ P NMR $\left(162 \mathrm{MHz}, \mathrm{CDCl}_{3}\right) \delta 23.8$; HRMS (ESI) $m / z$ calc'd for $\mathrm{C}_{21} \mathrm{H}_{19} \mathrm{ON}_{2} \mathrm{ClP}[\mathrm{M}+\mathrm{H}]^{+}$: 381.0918, found: 381.0916; [ $\alpha]_{\mathrm{D}^{24}} 19.1$ (c 0.10, $\mathrm{CHCl}_{3}, 97 \%$ ee sample); HPLC (CHIRALCEL IC-3 ( $\left.\phi 0.46 \mathrm{~cm} \times 25 \mathrm{~cm}\right), n$ hexane/2-propanol = 2/1, flow rate $1.0 \mathrm{~mL} / \mathrm{min}$, detection at $254 \mathrm{~nm}, \mathrm{t}_{\mathrm{R}}=10.1 \mathrm{~min}$ (minor), $11.8 \mathrm{~min}$ (major).

Racemic sample

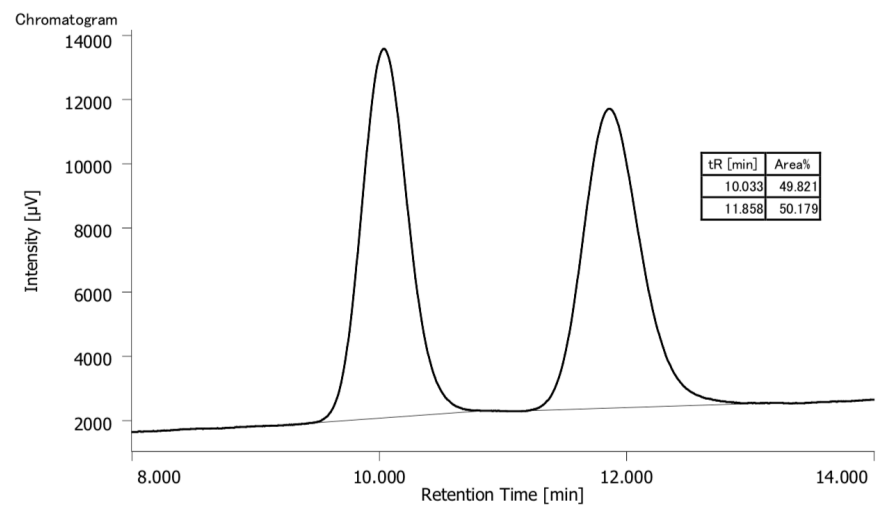

Reaction sample

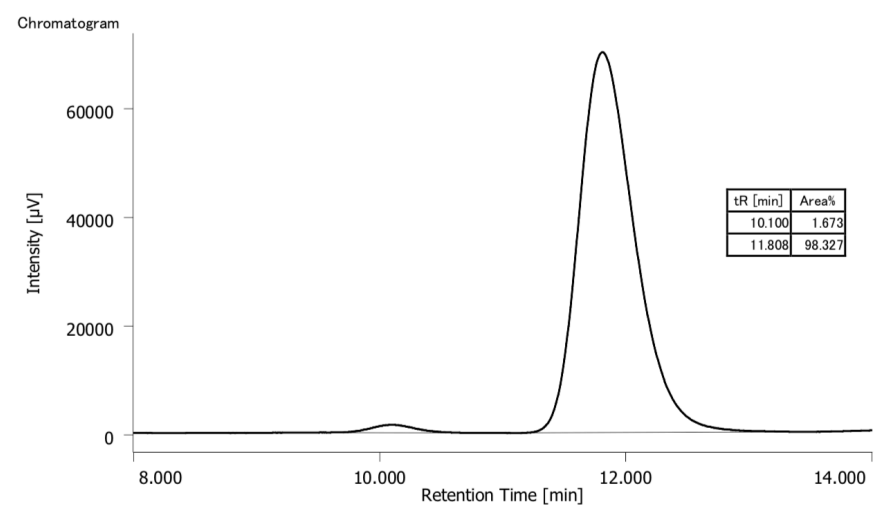


(R)-N-(2-Cyano-1-(4-bromophenyl)ethyl)-P,P-diphenylphosphinic amide (2g): Prepared by the general procedure<smiles>N#CCC(NP(=O)(F)F)c1ccc(Br)cc1</smiles>
from $1 \mathrm{~g}(38.5 \mathrm{mg}, 0.100 \mathrm{mmol})$ and $5 \mathrm{~mol} \%$ catalyst, and isolated as a white solid $(40.8 \mathrm{mg}, 96 \%)$. m.p. $166-168^{\circ} \mathrm{C}$; IR $\left(\mathrm{CHCl}_{3}\right): 3159,2894,2251,1592,1488,1439,1181,1124,1110,1091,1011 \mathrm{~cm}^{-1} ;{ }^{1} \mathbf{H}$ NMR $\left(400 \mathrm{MHz}, \mathrm{CDCl}_{3}\right) \delta 7.91-7.79(\mathrm{~m}, 4 \mathrm{H}), 7.58-7.42(\mathrm{~m}, 8 \mathrm{H}), 7.22(\mathrm{~d}, J=8.2 \mathrm{~Hz}, 2 \mathrm{H}), 4.59-4.51(\mathrm{~m}$, $1 \mathrm{H}), 3.54(\mathrm{dd}, J=10.8,6.7 \mathrm{~Hz}, 1 \mathrm{H}), 3.08(\mathrm{dd}, J=16.9,7.1 \mathrm{~Hz}, 1 \mathrm{H}), 2.92(\mathrm{dd}, J=16.9,4.3 \mathrm{~Hz}, 1 \mathrm{H}) ;{ }^{13} \mathrm{C}$ NMR $\left(100 \mathrm{MHz}, \mathrm{CDCl}_{3}\right) \delta 132.42(\mathrm{~d}, J=2.6 \mathrm{~Hz}), 132.37(\mathrm{~d}, J=2.9 \mathrm{~Hz}), 132.25(\mathrm{~d}, J=11.2 \mathrm{~Hz}), 132.21$, $132.0(\mathrm{~d}, J=58.7 \mathrm{~Hz}), 131.7(\mathrm{~d}, J=9.8 \mathrm{~Hz}), 130.7(\mathrm{~d}, J=58.7 \mathrm{~Hz}), 128.8(\mathrm{~d}, J=2.1 \mathrm{~Hz}), 128.7(\mathrm{~d}, J=2.0$ $\mathrm{Hz}), 127.8,122.5,116.9,51.0,28.0(\mathrm{~d}, J=3.2 \mathrm{~Hz})$; ${ }^{31} \mathbf{P}$ NMR $\left(162 \mathrm{MHz}, \mathrm{CDCl}_{3}\right) \delta 23.9$; HRMS (ESI) $\mathrm{m} / z$ calc'd for

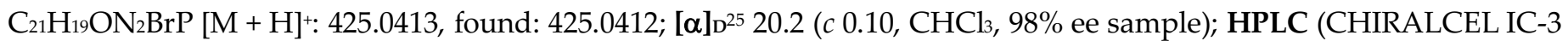
$(\phi 0.46 \mathrm{~cm} \times 25 \mathrm{~cm}), n$-hexane/2-propanol = 2/1, flow rate $1.0 \mathrm{~mL} / \mathrm{min}$, detection at $254 \mathrm{~nm}, \mathrm{t}_{\mathrm{R}}=10.3 \mathrm{~min}$ (minor), $12.1 \mathrm{~min}$ (major).

Racemic sample

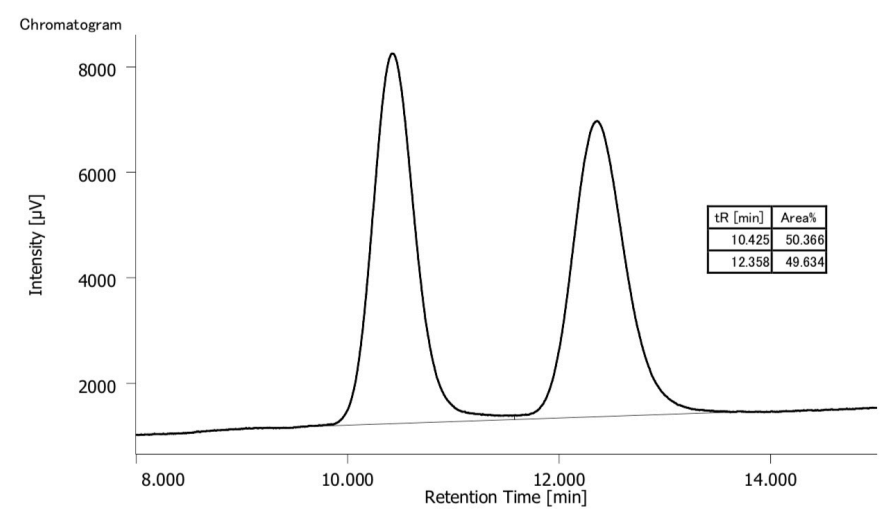

Reaction sample

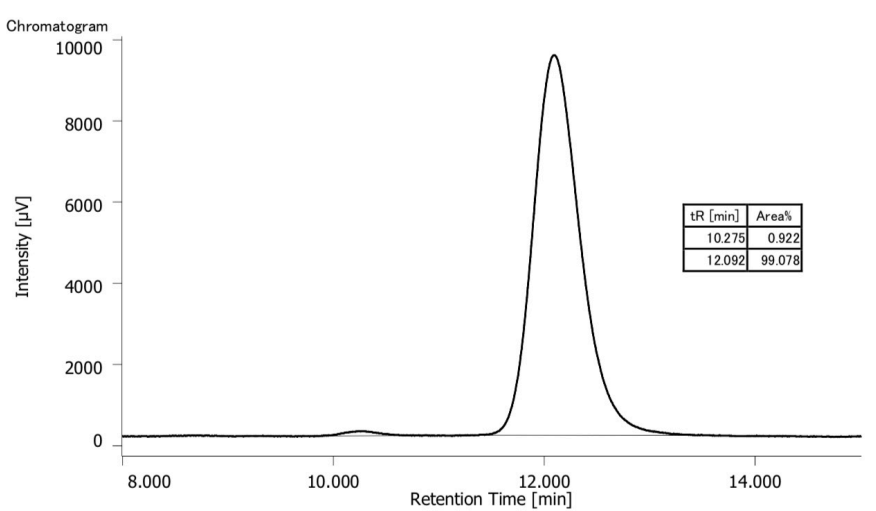

(R)-N-(2-Cyano-1-(4-(trifluoromethyl)phenyl)ethyl)- $P, P$-diphenylphosphinic amide (2h): Prepared by the general<smiles>N#CCC(NP=O)c1ccc(C(F)(F)F)cc1</smiles>
procedure from $1 \mathrm{~h}(37.3 \mathrm{mg}, 0.100 \mathrm{mmol})$ and $2 \mathrm{~mol} \%$ catalyst, and isolated as a white solid (39.9 mg, 96\%). m.p. 190-192 ${ }^{\circ} \mathrm{C}$; IR ( $\left.\mathrm{CHCl}_{3}\right): 3166,2901,2248,1619,1592,1463,1438,1421,1405,1329$, $1178,1115,1069,1019 \mathrm{~cm}^{-1} ;{ }^{1} \mathrm{H}$ NMR $\left(400 \mathrm{MHz}, \mathrm{CDCl}_{3}\right) \delta 7.91-7.77(\mathrm{~m}, 4 \mathrm{H}), 7.64(\mathrm{~d}, J=8.1 \mathrm{~Hz}, 2 \mathrm{H})$, 7.58-7.39 (m, 8H), 4.68-4.58 (m, 1H), $3.80(\mathrm{dd}, J=11.0,6.5 \mathrm{~Hz}, 1 \mathrm{H}), 3.10(\mathrm{dd}, J=16.7,6.5 \mathrm{~Hz}, 1 \mathrm{H})$, $2.94(\mathrm{dd}, J=16.7,4.5 \mathrm{~Hz}, 1 \mathrm{H}) ;{ }^{13} \mathrm{C}$ NMR $\left(100 \mathrm{MHz}, \mathrm{CDCl}_{3}\right) \delta 143.8(\mathrm{~d}, J=5.7 \mathrm{~Hz}), 132.4(\mathrm{q}, J=2.7$ $\mathrm{Hz}), 132.2(\mathrm{~d}, J=9.9 \mathrm{~Hz}), 131.8(\mathrm{~d}, J=9.9 \mathrm{~Hz}), 131.7,130.8(\mathrm{~d}, J=70.9 \mathrm{~Hz}), 130.6(\mathrm{q}, J=32.5 \mathrm{~Hz})$, $130.5(\mathrm{~d}, J=72.6 \mathrm{~Hz}), 128.8(\mathrm{~d}, J=4.1 \mathrm{~Hz}), 128.7(\mathrm{~d}, J=4.1 \mathrm{~Hz}), 126.6,126.0(\mathrm{q}, J=3.7 \mathrm{~Hz}), 123.8(\mathrm{q}, J=271 \mathrm{~Hz}), 116.8,51.2$, $27.9(\mathrm{~d}, J=3.8 \mathrm{~Hz}) ;{ }^{19} \mathbf{F}$ NMR $\left(376 \mathrm{MHz}, \mathrm{CDCl}_{3}\right) \delta-62.7 ;{ }^{31} \mathbf{P}$ NMR $\left(162 \mathrm{MHz}, \mathrm{CDCl}_{3}\right) \delta 24.0$; HRMS (ESI) $m / z$ calc'd for $\mathrm{C}_{22} \mathrm{H}_{19} \mathrm{ON}_{2} \mathrm{~F}_{3} \mathrm{P}[\mathrm{M}+\mathrm{H}]^{+}: 415.1182$, found: 415.1186; [ $\left.\alpha\right]_{\mathrm{D}^{25}} 1.8$ (c 0.10, $\mathrm{CHCl}_{3}, 96 \%$ ee sample); HPLC (CHIRALCEL IA-3 ( $\phi$ $0.46 \mathrm{~cm} \times 25 \mathrm{~cm}$ ), $n$-hexane/2-propanol = 2/1, flow rate $1.0 \mathrm{~mL} / \mathrm{min}$, detection at $254 \mathrm{~nm}, \mathrm{t}_{\mathrm{R}}=11.1 \mathrm{~min}$ (minor), $12.3 \mathrm{~min}$ (major).

Racemic sample

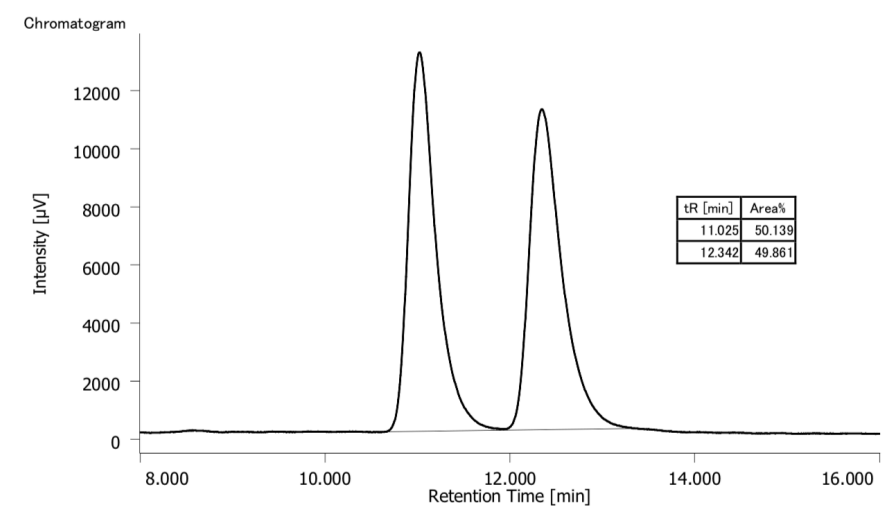

Reaction sample

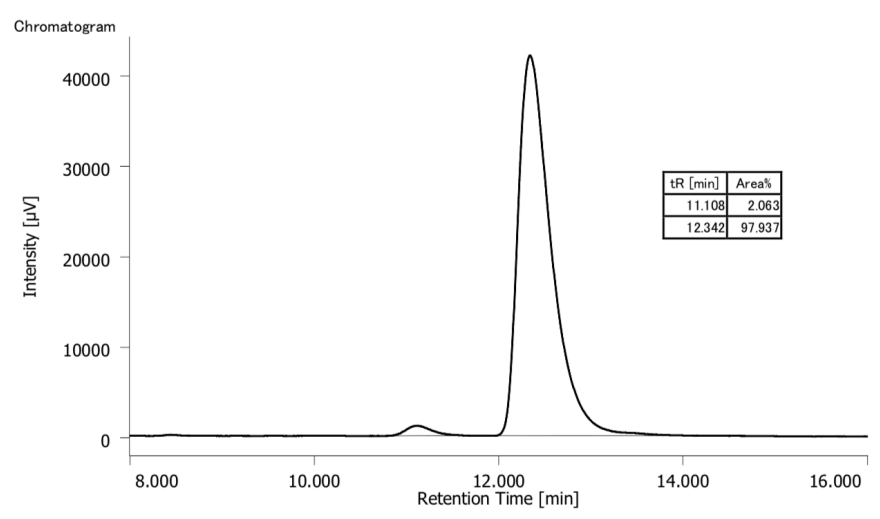


(R)-N-(2-Cyano-1-(4-cyanophenyl)ethyl)-P,P-diphenylphosphinic amide (2i): Prepared by the general procedure from<smiles>N#CCCc1ccc(NP)cc1</smiles>
$1 \mathbf{i}(33.0 \mathrm{mg}, 0.100 \mathrm{mmol})$ and $2 \mathrm{~mol} \%$ catalyst, and isolated as a white solid $(36.2 \mathrm{mg}, 98 \%)$. m.p. $84-$ $86^{\circ} \mathrm{C}$; IR $\left(\mathrm{CHCl}_{3}\right): 3149,2898,2251,2230,1609,1439,1416,1182,1124,1109 \mathrm{~cm}^{-1} ;{ }^{1} \mathrm{H}$ NMR $(400 \mathrm{MHz}$, $\left.\mathrm{CDCl}_{3}\right) \delta$ 7.91-7.78 (m, 4H), 7.70-7.66 (m, 2H), 7.60-7.40 (m, 8H), 4.69-4.59 (m, 1H), $3.65(\mathrm{dd}, J=10.9$, $6.2 \mathrm{~Hz}, 1 \mathrm{H}), 3.11(\mathrm{dd}, J=16.7,6.5 \mathrm{~Hz}, 1 \mathrm{H}), 2.93(\mathrm{dd}, J=16.7,4.4 \mathrm{~Hz}, 1 \mathrm{H}) ;{ }^{13} \mathrm{C} \mathbf{N M R}\left(100 \mathrm{MHz}, \mathrm{CDCl}_{3}\right)$ $\delta 145.0(\mathrm{~d}, J=5.6 \mathrm{~Hz}), 132.8,132.52(\mathrm{q}, J=2.8 \mathrm{~Hz}), 132.46(\mathrm{~d}, J=2.6 \mathrm{~Hz}), 132.1(\mathrm{~d}, J=9.7 \mathrm{~Hz}), 131.8(\mathrm{~d}$, $J=9.8 \mathrm{~Hz}), 131.8(\mathrm{~d}, J=32.7 \mathrm{~Hz}), 130.5(\mathrm{~d}, J=34.6 \mathrm{~Hz}), 128.8(\mathrm{q}, J=5.6 \mathrm{~Hz}), 128.7(\mathrm{~d}, J=5.6 \mathrm{~Hz}), 127.1$, 118.2, 116.6, 112.4, 51.3, $27.7(\mathrm{~d}, J=3.9 \mathrm{~Hz}) ;{ }^{31} \mathbf{P}$ NMR $\left(162 \mathrm{MHz}, \mathrm{CDCl}_{3}\right) \delta 24.2 ;$ HRMS (ESI) $m / z$ calc'd for $\mathrm{C}_{22} \mathrm{H}_{19} \mathrm{~N}_{3} \mathrm{OP}[\mathrm{M}$ $+\mathrm{H}]^{+}:$372.1260, found: 372.1260; [ $\left.\alpha\right]_{\mathrm{D}^{25}} 43.8$ ( $c$ 0.15, $\mathrm{CHCl}_{3}, 94 \%$ ee sample); HPLC (CHIRALCEL IA-3 $(\phi 0.46 \mathrm{~cm} \times 25 \mathrm{~cm})$, $n$-hexane $/ 2$-propanol = 2/1, flow rate $1.0 \mathrm{~mL} / \mathrm{min}$, detection at $254 \mathrm{~nm}, \mathrm{t}_{\mathrm{R}}=15.5 \mathrm{~min}$ (minor), $28.5 \mathrm{~min}$ (major).

Racemic sample

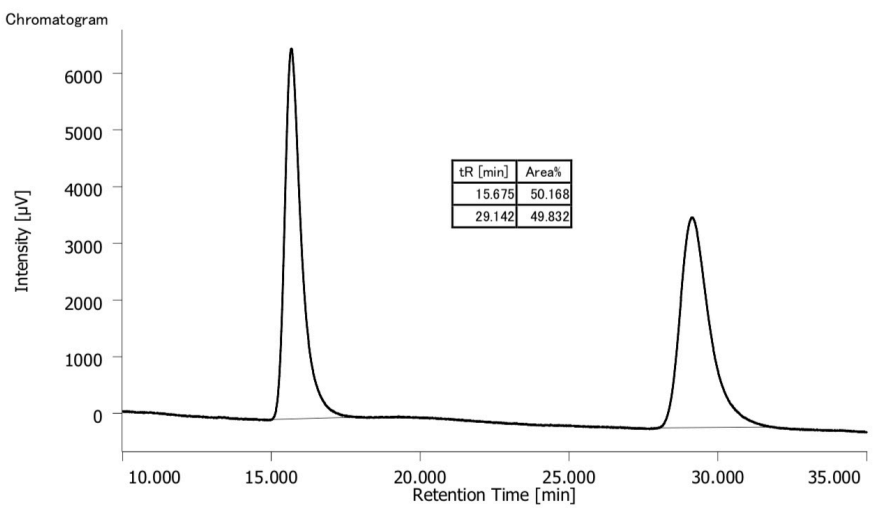

Reaction sample

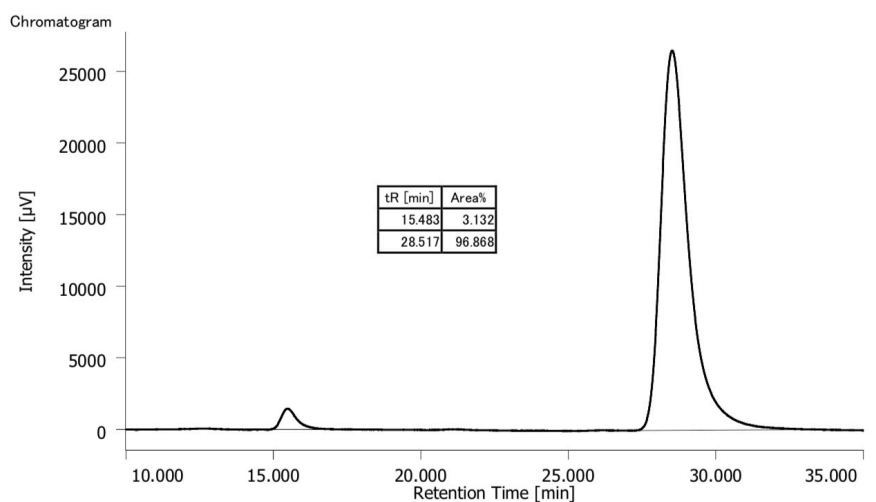

(R)-N-(2-Cyano-1-(furan-2-yl)ethyl)-P,P-diphenylphosphinic amide (2j): Prepared by the general procedure from $1 \mathbf{j}$<smiles>N#CCC(NP(=O)(O)O)c1ccco1</smiles>
(31.9 $\mathrm{mg}, 0.100 \mathrm{mmol}$ ) and $2 \mathrm{~mol} \%$ catalyst, and isolated as a white solid $(25.1 \mathrm{mg}, 75 \%)$. m.p. 120 $122{ }^{\circ} \mathrm{C}$; IR ( $\left.\mathrm{CHCl}_{3}\right): 3148,2890,2251,1592,1505,1439,1190,1124,1110,1072,1012 \mathrm{~cm}^{-1} ;{ }^{1} \mathrm{H}$ NMR $(400$ $\left.\mathrm{MHz}, \mathrm{CDCl}_{3}\right) \delta 7.97-7.83(\mathrm{~m}, 4 \mathrm{H}), 7.58-7.44(\mathrm{~m}, 6 \mathrm{H}), 7.40(\mathrm{dd}, J=1.8,0.8 \mathrm{~Hz}, 1 \mathrm{H}), 6.39(\mathrm{ddd}, J=3.3,0.7$, $0.7 \mathrm{~Hz}, 1 \mathrm{H}), 6.36(\mathrm{dd}, J=3.3,1.8 \mathrm{~Hz}, 1 \mathrm{H}), 4.67-4.57(\mathrm{~m}, 1 \mathrm{H}), 3.60(\mathrm{dd}, J=11.2,7.6 \mathrm{~Hz}, 1 \mathrm{H}), 3.11(\mathrm{dd}, J=$ $16.6,6.5 \mathrm{~Hz}, 1 \mathrm{H}), 3.04(\mathrm{dd}, J=16.6,4.5 \mathrm{~Hz}, 1 \mathrm{H}) ;{ }^{13} \mathrm{C}$ NMR $\left(100 \mathrm{MHz}, \mathrm{CDCl}_{3}\right) \delta 152.0(\mathrm{~d}, J=8.3 \mathrm{~Hz}), 142.8$, $132.40(\mathrm{~d}, J=9.9 \mathrm{~Hz}), 132.37,132.0(\mathrm{~d}, J=71.5 \mathrm{~Hz}), 131.7(\mathrm{~d}, J=9.9 \mathrm{~Hz}), 130.7(\mathrm{~d}, J=71.6 \mathrm{~Hz}), 128.8(\mathrm{~d}, J=4.2 \mathrm{~Hz}), 128.7(\mathrm{~d}$, $J=4.2 \mathrm{~Hz}), 116.8,110.6,107.6,46.2,26.1(\mathrm{~d}, J=2.7 \mathrm{~Hz}) ;{ }^{31} \mathbf{P}$ NMR $\left(162 \mathrm{MHz}, \mathrm{CDCl}_{3}\right) \delta 23.8$; HRMS (ESI) $\mathrm{m} / z$ calc'd for $\mathrm{C}_{19} \mathrm{H}_{18} \mathrm{~N}_{2} \mathrm{O}_{2} \mathrm{P}[\mathrm{M}+\mathrm{H}]^{+}: 337.1100$, found: 337.1108 ; [ $\left.\alpha\right]_{\mathrm{D}^{25}} 13.8$ (c $0.11, \mathrm{CHCl}_{3}, 90 \%$ ee sample); HPLC (CHIRALCEL IC-3 ( $\phi$ $0.46 \mathrm{~cm} \times 25 \mathrm{~cm}$ ), $n$-hexane/2-propanol =2/1, flow rate $1.0 \mathrm{~mL} / \mathrm{min}$, detection at $254 \mathrm{~nm}, \mathrm{t}_{\mathrm{R}}=13.4 \mathrm{~min}(\operatorname{minor}), 14.6 \mathrm{~min}$ (major).

Racemic sample

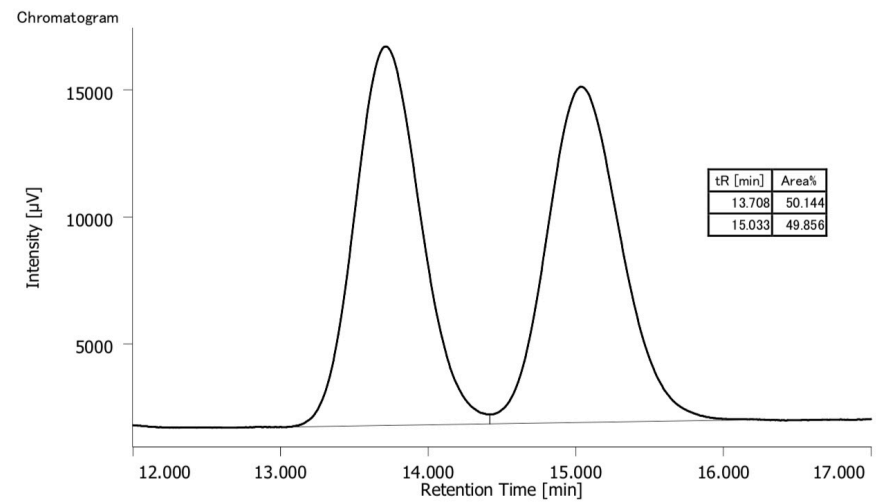

Reaction sample

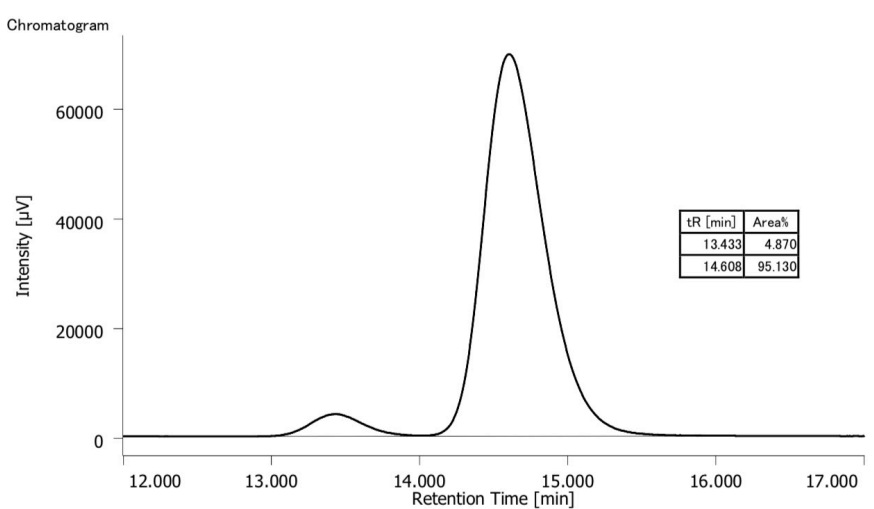


(R)-N-(2-Cyano-1-(thiophen-2-yl)ethyl)-P,P-diphenylphosphinic amide (2k): Prepared by the general procedure from<smiles>N#CCC(NP(=O)=O)c1cccs1</smiles>

1k (31.1 mg, $0.100 \mathrm{mmol})$ and $2 \mathrm{~mol} \%$ catalyst, and isolated as a white solid (33.4 mg, 95\%). m.p. 152$154{ }^{\circ} \mathrm{C}$; IR $\left(\mathrm{CHCl}_{3}\right): 3122,2874,2251,1591,1462,1439,1416,1306,1189,1123,1109,1093 \mathrm{~cm}^{-1} ;{ }^{1} \mathbf{H}$ NMR $\left(400 \mathrm{MHz}, \mathrm{CDCl}_{3}\right) \delta$ 7.99-7.85 (m, 4H), 7.56-7.51 (m, 2H), 7.51-7.44 (m, 4H), 7.29 (dd, J = 5.1, 1.2 Hz, $\left.1 \mathrm{H}\right)$, $7.07(\mathrm{ddd}, J=3.6,1.2,1.2 \mathrm{~Hz}, 1 \mathrm{H}), 7.00(\mathrm{dd}, J=5.1,3.6 \mathrm{~Hz}, 1 \mathrm{H}), 4.84-4.75(\mathrm{~m}, 1 \mathrm{H}), 3.80-3.72(\mathrm{~m}, 1 \mathrm{H}), 3.21$ $(\mathrm{ddd}, J=16.7,6.4,1.3 \mathrm{~Hz}, 1 \mathrm{H}), 3.08(\mathrm{dd}, J=16.7,3.8 \mathrm{~Hz}, 1 \mathrm{H}) ;{ }^{13} \mathrm{C}$ NMR $\left(100 \mathrm{MHz}, \mathrm{CDCl}_{3}\right) \delta 143.7(\mathrm{~d}, J=$ $8.7 \mathrm{~Hz}), 132.4(\mathrm{~d}, J=9.6 \mathrm{~Hz}), 131.6(\mathrm{~d}, J=9.8 \mathrm{~Hz}), 131.3(\mathrm{~d}, J=39.7 \mathrm{~Hz}), 130.2,128.8(\mathrm{~d}, J=3.0 \mathrm{~Hz}), 128.7(\mathrm{~d}, J=3.0 \mathrm{~Hz})$, 127.3, 125.4, 124.9, 116.9, 47.8, $28.4(\mathrm{~d}, J=1.9 \mathrm{~Hz}) ;{ }^{31 P} \mathrm{NMR}\left(162 \mathrm{MHz}, \mathrm{CDCl}_{3}\right) \delta 23.4$; HRMS (ESI) $\mathrm{m} / z$ calc'd for $\mathrm{C}_{19} \mathrm{H}_{18} \mathrm{~N}_{2} \mathrm{OPS}[\mathrm{M}+\mathrm{H}]^{+}: 353.0872$, found: 353.0878; $[\alpha]_{\mathrm{D}^{24}}-8.8\left(c 0.10, \mathrm{CHCl}_{3}, 98 \%\right.$ ee sample); HPLC (CHIRALCEL IC-3 $(\phi$ $0.46 \mathrm{~cm} \times 25 \mathrm{~cm}$ ), $n$-hexane/2-propanol =2/1, flow rate $1.0 \mathrm{~mL} / \mathrm{min}$, detection at $254 \mathrm{~nm}, \mathrm{tr}_{\mathrm{R}}=13.2 \mathrm{~min}$ (minor), $14.8 \mathrm{~min}$ (major).

Racemic sample

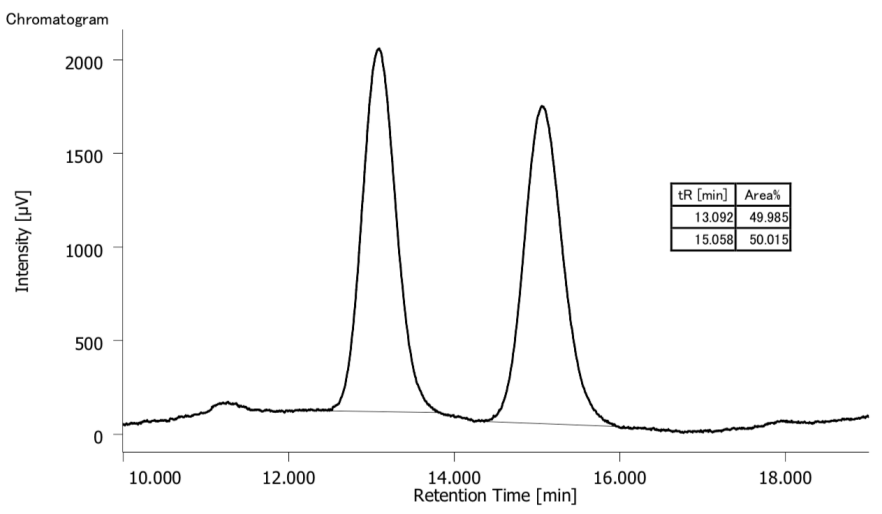

Reaction sample

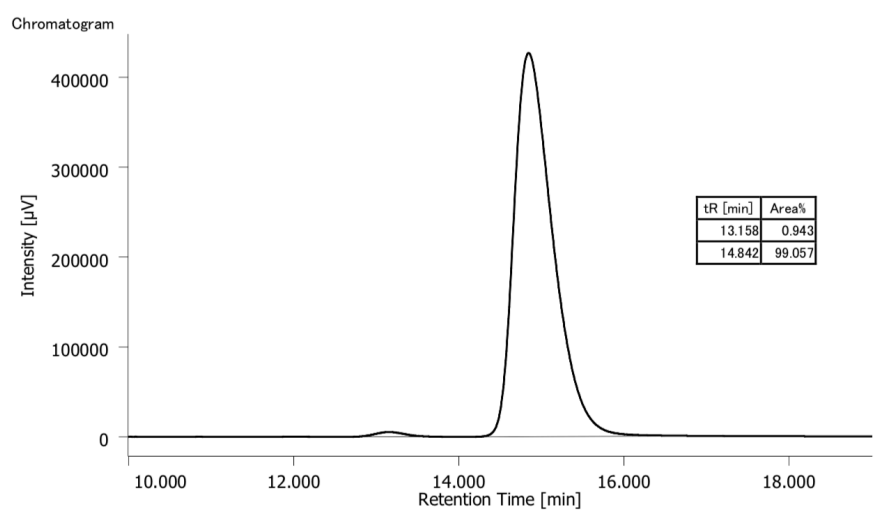

$(R, E)-N$-(1-Cyano-4-phenylbut-3-en-2-yl)-P,P-diphenylphosphinic amide (21): Prepared by the general procedure from $11(33.1 \mathrm{mg}, 0.100 \mathrm{mmol})$ and $5 \mathrm{~mol} \%$ catalyst, and isolated as a white solid $(37.2 \mathrm{mg}, 93 \%)$. m.p.<smiles>N#CCC/C=C/c1ccccc1</smiles>
93-95 ${ }^{\circ} \mathrm{C}$; IR ( $\left.\mathrm{CHCl}_{3}\right): 3151,2887,2250,1592,1495,1448,1439,1187,1124,1110,1071 \mathrm{~cm}^{-1}$; ${ }^{1} \mathbf{H ~ N M R}$ $\left(400 \mathrm{MHz}, \mathrm{CDCl}_{3}\right) \delta$ 7.97-7.85 (m, 4H), 7.57-7.51 (m, 2H), 7.50-7.44 (m, 4H), 7.38-7.25 (m, 5H), 6.62 $(\mathrm{d}, J=15.9 \mathrm{~Hz}, 1 \mathrm{H}), 6.24(\mathrm{dd}, J=15.9,6.3 \mathrm{~Hz}, 1 \mathrm{H}), 4.25-4.14(\mathrm{~m}, 1 \mathrm{H}), 3.44-3.34(\mathrm{~m}, 1 \mathrm{H}), 2.96(\mathrm{dd}, J=$ 16.6, $6.1 \mathrm{~Hz}, 1 \mathrm{H}), 2.80(\mathrm{dd}, J=16.6,4.1 \mathrm{~Hz}, 1 \mathrm{H}) ;{ }^{13} \mathrm{C} \mathrm{NMR}\left(100 \mathrm{MHz}, \mathrm{CDCl}_{3}\right) \delta 135.6,132.5,132.4(\mathrm{~d}$, $J=60.5 \mathrm{~Hz}), 132.33(\mathrm{~d}, J=9.5 \mathrm{~Hz}), 132.31,131.7(\mathrm{~d}, J=9.8 \mathrm{~Hz}), 131.1(\mathrm{~d}, J=63.2 \mathrm{~Hz}), 128.8(\mathrm{~d}, J=3.1$ $\mathrm{Hz}), 128.7(\mathrm{~d}, J=3.1 \mathrm{~Hz}), 128.6,128.3,127.3(\mathrm{~d}, J=7.1 \mathrm{~Hz}), 126.7,117.2,49.7,27.2(\mathrm{~d}, J=2.8 \mathrm{~Hz})$; ${ }^{31 P} \mathrm{NMR}(162 \mathrm{MHz}$, $\left.\mathrm{CDCl}_{3}\right) \delta$ 23.7; HRMS (ESI) $\mathrm{m} / z$ calc'd for $\mathrm{C}_{23} \mathrm{H}_{22} \mathrm{~N}_{2} \mathrm{OP}[\mathrm{M}+\mathrm{H}]^{+}: 373.1464$, found: $373.1467 ;[\alpha]_{\mathrm{D}^{24}} 60.2\left(\mathrm{c} 0.22, \mathrm{CHCl}_{3}, 95 \%\right.$ ee sample); HPLC (CHIRALCEL IC-3 $(\phi 0.46 \mathrm{~cm} \times 25 \mathrm{~cm}), n$-hexane $/ 2$-propanol = 2/1, flow rate $1.0 \mathrm{~mL} / \mathrm{min}$, detection at $254 \mathrm{~nm}, \mathrm{t}_{\mathrm{R}}=11.5 \mathrm{~min}$ (minor), $14.5 \mathrm{~min}$ (major).

Racemic sample

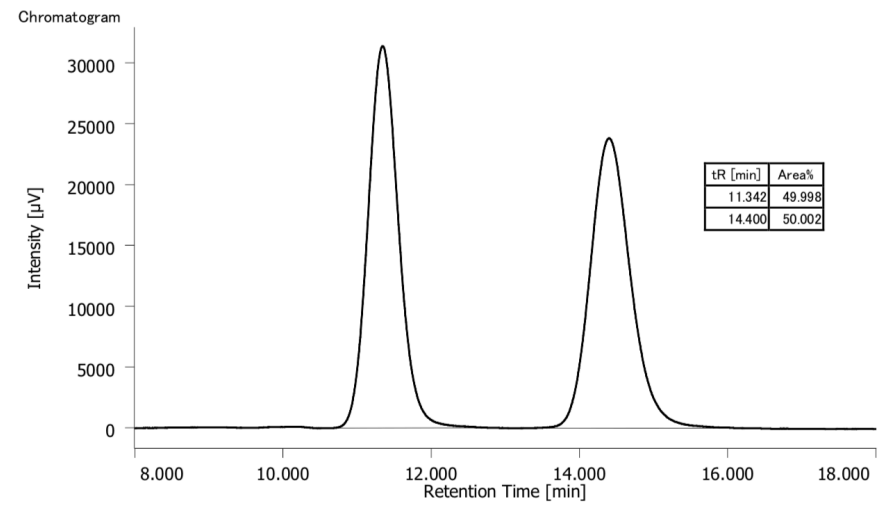

Reaction sample

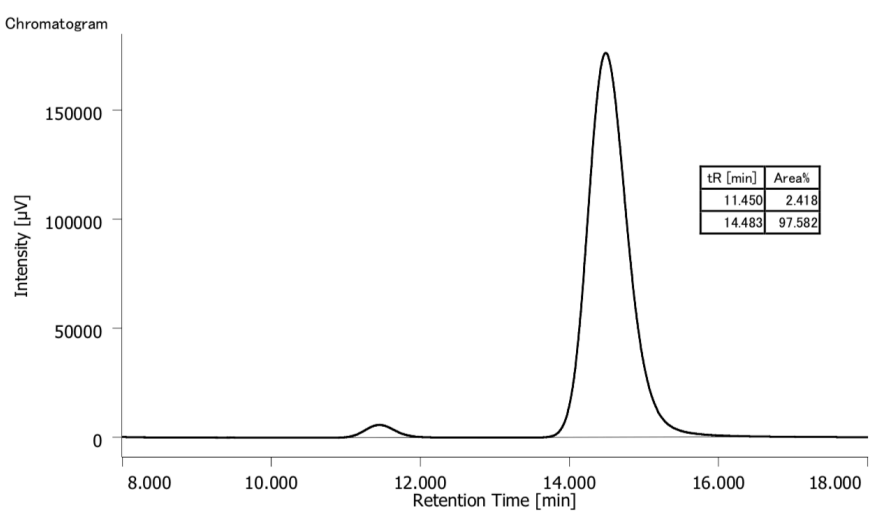




\section{3-4. Large-scale synthesis}

A flame-dried round-bottom flask was charged with $\mathrm{Ni} / \mathrm{L} 2(57.6 \mathrm{mg}, 0.080 \mathrm{mmol})$ and ${ }^{t} \mathrm{BuOK}(9.0 \mathrm{mg}, 0.080 \mathrm{mmol})$ in a dry box. After the test tube was cooled down to $0{ }^{\circ} \mathrm{C}, \mathrm{CH}_{3} \mathrm{CN}(8.0 \mathrm{~mL})$ was added, and the resulting solution was stirred for $10 \mathrm{~min}$ to afford $0.01 \mathrm{M} \mathrm{Ni}(\mathrm{II})$ catalyst solution. The catalyst solution was quickly used within $1 \mathrm{~h}$ after preparation. A solution of $1 \mathrm{a}(1.20 \mathrm{~g}, 3.93 \mathrm{mmol})$ in $\mathrm{CH}_{3} \mathrm{CN}(11.8 \mathrm{~mL})$ was cooled to $0{ }^{\circ} \mathrm{C}$, and the $\mathrm{Ni}(\mathrm{II})$ catalyst solution $(7.86 \mathrm{~mL}$, $0.0786 \mathrm{mmol}, 2 \mathrm{~mol} \%$ ) was slowly added. The resulting mixture was stirred for $24 \mathrm{~h}$ at $0{ }^{\circ} \mathrm{C}$, and quenched with $0.1 \mathrm{M} 4$ $0 \mathrm{M} \mathrm{HCl} / 1$,4-dioxane solution $(1.0 \mathrm{~mL})$. After the addition of sat. $\mathrm{NaHCO}_{3}$ aqueous solution, the mixture was extracted with AcOEt, and the combined organic layers were washed with brine, dried over $\mathrm{Na}_{2} \mathrm{SO}_{4}$, and concentrated in vacuo. The residue was purified with silica gel column chromatography $\left(\mathrm{CHCl}_{3} / \mathrm{MeOH}=95 / 5\right)$ to afford the aminonitrile $2 \mathrm{a}$ $(1.26 \mathrm{~g}, 93 \%)$ Enantiomeric excess was determined to be $97 \%$.

\section{3-5. Determination of absolute configuration of the products}

The absolute configuration of the product 2a was determined after converting to the known compound (3) by the following procedure described in the next section. ${ }^{6}$ The absolute configuration of the other products was deduced by analogy.

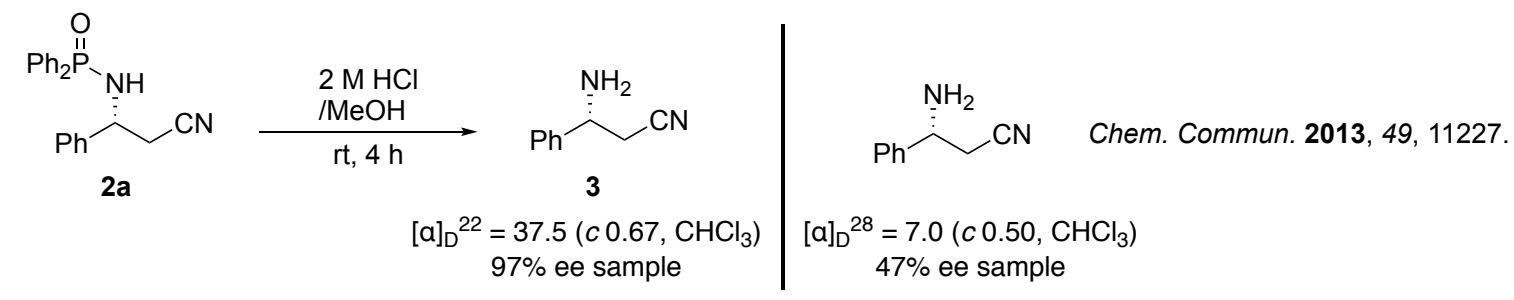

\section{Transformation of the Mannich Adduct}

(R)-3-Amino-3-phenylpropanenitrile (3):

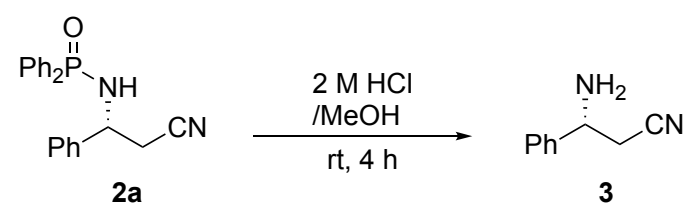

To $2 \mathrm{a}(34.6 \mathrm{mg}, 0.100 \mathrm{mmol})$ in a test tube was added $\mathrm{HCl}$ solution in $\mathrm{MeOH}(c a .2 .0 \mathrm{M}, 5.0 \mathrm{~mL})$ at room temperature, and the resulting mixture was stirred at the same temperature for $4 \mathrm{~h}$. After evaporation of solvent, the residue was dissolved in $1 \mathrm{M} \mathrm{HCl}$ aqueous solution and the resulting aqueous mixture was washed with $\mathrm{Et}_{2} \mathrm{O}$ to remove $\mathrm{Ph}_{2} \mathrm{P}(\mathrm{O}) \mathrm{OMe}$. To the mixture was added sat. $\mathrm{NaHCO}_{3}$ aq. until the solution became cloudy, and the resulting suspension was extracted with $\mathrm{CH}_{2} \mathrm{Cl}_{2}$. The combined organic layers were dried over $\mathrm{Na}_{2} \mathrm{SO}_{4}$, and concentrated in vacuo to afford $3(13.4 \mathrm{mg}, 92 \%)$

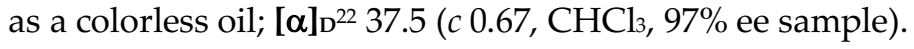

\section{(R)-3-((Diphenylphosphoryl)amino)-3-phenylpropanoic acid (4):}<smiles>N#CCC(Nc1ccccc1)c1ccccc1</smiles><smiles>CC(CC(=O)O)NP(=O)(O)c1ccccc1</smiles>

To a solution of $\mathbf{2 a}(34.6 \mathrm{mg}, 0.100 \mathrm{mmol})$ in $\mathrm{EtOH}(1.0 \mathrm{~mL})$ was added aqueous $\mathrm{NaOH}$ solution $(2.0 \mathrm{M}, 500 \mu \mathrm{L})$, and the resulting mixture was stirred under reflux condition for $16 \mathrm{~h}$. After cooling to room temperature, the volatiles were removed under reduced pressure, and the resulting suspension was washed with $\mathrm{Et}_{2} \mathrm{O}$. To the suspension was added 1 $\mathrm{M} \mathrm{HCl}$ aqueous solution to acidify, and the mixture was extracted with $\mathrm{CH}_{2} \mathrm{Cl}_{2}$. The combined organic layers were dried over $\mathrm{Na}_{2} \mathrm{SO}_{4}$, and concentrated in vacuo to afford $4(34.9 \mathrm{mg}, 96 \%)$ as an white solid.

m.p. $184-186^{\circ} \mathrm{C}$; IR $\left(\mathrm{CHCl}_{3}\right): 3262,2898,2626,2592,2567,1721,1438,1395,1128,1109,1080,1026 \mathrm{~cm}^{-1}$; ${ }^{1} \mathrm{H}$ NMR $(400$ MHz, $\left.\mathrm{CDCl}_{3}\right) \delta$ 7.90-7.70 (m, 3H), 7.53-7.20 (m, 12H), 4.63-4.46 (m, 2H), $3.09(\mathrm{dd}, J=16.1,5.0 \mathrm{~Hz}, 1 \mathrm{H}), 2.93(\mathrm{dd}, J=16.1$, $5.4 \mathrm{~Hz}, 1 \mathrm{H}) ;{ }^{13} \mathrm{C}$ NMR $\left(100 \mathrm{MHz}, \mathrm{CDCl}_{3}\right) \delta 173.1,142.4(\mathrm{~d}, J=7.4 \mathrm{~Hz}), 132.6(\mathrm{~d}, J=9.8 \mathrm{~Hz}), 132.11(\mathrm{~d}, J=3.3 \mathrm{~Hz}), 132.08(\mathrm{~d}$, $J=3.0 \mathrm{~Hz}), 131.8(\mathrm{~d}, J=9.7 \mathrm{~Hz}), 130.9(\mathrm{~d}, J=64.0 \mathrm{~Hz}), 129.0(\mathrm{~d}, J=64.0 \mathrm{~Hz}), 128.6(\mathrm{~d}, J=3.3 \mathrm{~Hz}), 128.44(\mathrm{~d}, J=3.7 \mathrm{~Hz})$, 128.42, 127.2, 126.4, 52.3, $42.9(\mathrm{~d}, J=2.6 \mathrm{~Hz}) ;{ }^{31} \mathbf{P}$ NMR $\left(162 \mathrm{MHz}, \mathrm{CDCl}_{3}\right) \delta$ 25.6; HRMS (ESI) $m / z$ calc'd for $\mathrm{C}_{21} \mathrm{H}_{21} \mathrm{NO}_{3} \mathrm{P}$ $[\mathrm{M}+\mathrm{H}]^{+}: 366.1245$, found: $366.1248 ;[\alpha]_{\mathrm{D}^{24}} 10.6$ ( $0.10, \mathrm{CHCl}_{3}, 97 \%$ ee sample). 
(R)-N-(3-Amino-1-phenylpropyl)-P,P-diphenylphosphinic amide (5):<smiles>N#CCC(NP(=O)=O)c1ccccc1</smiles>

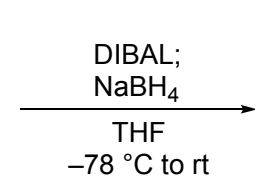

$-78^{\circ} \mathrm{C}$ to rt<smiles>NCCC(Nc1ccccc1)c1ccccc1</smiles>

5

To a solution of $2 \mathrm{a}(34.6 \mathrm{mg}, 0.100 \mathrm{mmol})$ in THF $(1.0 \mathrm{~mL})$ was added DIBAL $(1.0 \mathrm{M}$ in toluene solution, $500 \mu \mathrm{L}, 0.500$ $\mathrm{mmol})$ at $-78{ }^{\circ} \mathrm{C}$, and the resulting mixture was stirred for $2 \mathrm{~h}$ at the same temperature. After addition of $\mathrm{NaBH}_{4}(37.8$ $\mathrm{mg}, 1.00 \mathrm{mmol}$ ), the cooling bath was removed and the mixture was stirred for $16 \mathrm{~h}$. To the mixture were successively added sat. $\mathrm{NH}_{4} \mathrm{Cl}$ aqueous solution and sat. Rochelle's salt aqueous solution. After stirring for $2 \mathrm{~h}$, the mixture was extracted with AcOEt, and the combined organic layers were dried over $\mathrm{Na}_{2} \mathrm{SO}_{4}$ and concentrated in vacuo. The residue was purified by preparative TLC (silica gel, $n$-hexane/AcOEt $=1 / 2)$ to afford $5(25.3 \mathrm{mg}, 72 \%)$ as a colorless oil.

IR ( $\left.\mathrm{CHCl}_{3}\right): 3222,3137,2364,2316,1604,1592,1456,1439,1168,1125,1108,1071 \mathrm{~cm}^{-1}$; 1 H NMR $\left(400 \mathrm{MHz}, \mathrm{CDCl}_{3}\right) \delta 7.83-$ $7.73(\mathrm{~m}, 2 \mathrm{H}), 7.67-7.61(\mathrm{~m}, 2 \mathrm{H}), 7.54-7.39(\mathrm{~m}, 4 \mathrm{H}), 7.34-7.24(\mathrm{~m}, 5 \mathrm{H}), 7.17-7.12(\mathrm{~m}, 2 \mathrm{H}), 5.11$ (brs, $1 \mathrm{H}), 4.74(\mathrm{brs}, 1 \mathrm{H}), 4.30$ (ddd, $J=22.3,11.1,4.2 \mathrm{~Hz}, 1 \mathrm{H}), 3.55(\mathrm{dd}, J=11.1,9.4 \mathrm{~Hz}, 1 \mathrm{H}), 3.20-3.08(\mathrm{~m}, 1 \mathrm{H}), 3.01-2.91(\mathrm{~m}, 1 \mathrm{H}), 2.28-2.17(\mathrm{~m}, 1 \mathrm{H})$, 2.11-2.00 (m, 1H); ${ }^{13} \mathrm{C}$ NMR $\left(100 \mathrm{MHz}, \mathrm{CDCl}_{3}\right) \delta 143.5(\mathrm{~d}, J=5.7 \mathrm{~Hz}), 132.8(\mathrm{~d}, J=10.1 \mathrm{~Hz}), 132.4(\mathrm{~d}, J=2.9 \mathrm{~Hz}), 132.22(\mathrm{~d}$, $J=2.9 \mathrm{~Hz}), 132.16(\mathrm{~d}, J=125.8 \mathrm{~Hz}), 130.3(\mathrm{~d}, J=132.0 \mathrm{~Hz}), 128.77,128.75(\mathrm{~d}, J=12.7 \mathrm{~Hz}), 128.5(\mathrm{~d}, J=12.9 \mathrm{~Hz}), 127.5,125.8$, 52.9, 44.6, $36.3(\mathrm{~d}, J=2.2 \mathrm{~Hz}) ;{ }^{31} \mathrm{P}$ NMR $\left(162 \mathrm{MHz}, \mathrm{CDCl}_{3}\right) \delta 26.9 ;$ HRMS (ESI) $\mathrm{m} / z$ calc'd for $\mathrm{C}_{21} \mathrm{H}_{24} \mathrm{NO}_{2} \mathrm{P}[\mathrm{M}+\mathrm{H}]^{+}: 351.1621$, found: $351.1621 ;[\alpha]_{\mathrm{D}^{25}} 14.8$ (c $0.41, \mathrm{CHCl}_{3}, 97 \%$ ee sample). 


\section{Solid State Structure of Ni/L2}

Single crystals of $\mathrm{Ni} / \mathrm{L} 2(\mathrm{X}=\mathrm{Cl})$ were obtained by slow evaporation from solution in $n$-hexane and dichloromethane at room temperature. A suitable crystal was selected and the sample was measured on a Rigaku R-AXIS RAPID imaging plate area detector with graphite-monochromated $\mathrm{Cu}-\mathrm{K} \alpha$ radiation. The data were collected at $93 \mathrm{~K}$. Refined structure and crystallographic parameters are summarized in Supplementary Table S1 and Supplementary Fig. S1. The ORTEP diagram was drawn by OLEX ${ }^{2} .7$ CCDC 1922993 contains the supplementary crystallographic data for Ni/L2 $(\mathrm{X}=\mathrm{Cl})$.

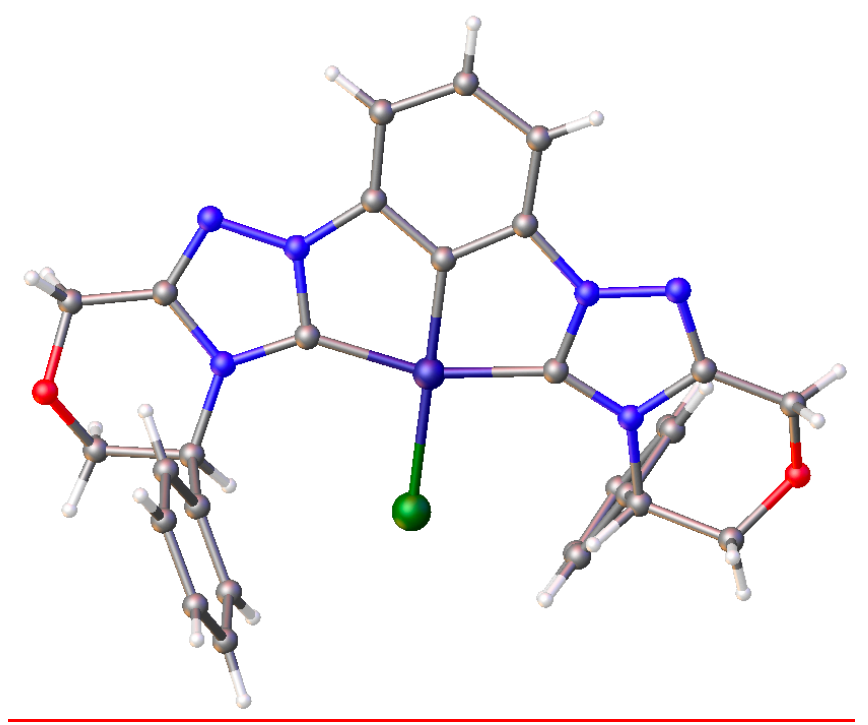

Figure S1. Structure of Ni/L2 $(X=\mathrm{Cl})$. Color code; White: hydrogen, gray: carbon, blue: nitrogen, red: oxygen, dark blue: nickel, dark green: chlorine.
Supplementary Table S1. Selected crystallographic data of $\mathrm{Ni} / \mathrm{L} 2(X=\mathrm{Cl})$.

Empirical Formula

Formula Weight

Crystal Color, Habit

Crystal Dimensions

Crystal System

Lattice Parameters

\author{
$\mathrm{C}_{28} \mathrm{H}_{23} \mathrm{ClN}_{6} \mathrm{NiO}_{2}$ \\ 569.68 \\ orange, block \\ $0.300 \times 0.200 \times 0.050 \mathrm{~mm}$ \\ orthorhombic
}

$6.68270(10) \AA$
$18.5119(4) \AA$
$19.4836(4) \AA$
$2410.31(7) \AA^{3}$
$C 2221$
4
$1.570 \mathrm{~g} / \mathrm{cm}^{3}$
1176.00
$0.064(12)$

$6.68270(10) \AA$

b $18.5119(4) \AA$

c $19.4836(4) \AA$

$\mathrm{V}$

Space Group

$Z$ value

$0.064(12)$ 


\section{Kinetic Isotope Effect}

\section{6-1. One-pot reaction}

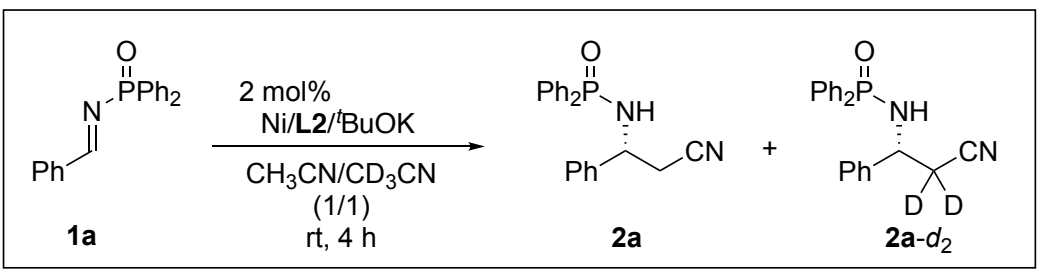

A flame-dried test tube $(20 \mathrm{~mL})$ was charged with $\mathrm{Ni} / \mathrm{L} 2(7.2 \mathrm{mg}, 0.010 \mathrm{mmol})$ and ${ }^{\mathrm{BuOK}}(1.1 \mathrm{mg}, 0.010 \mathrm{mmol}) \mathrm{in}$ a dry box. After the test tube was cooled down to $0{ }^{\circ} \mathrm{C}$, THF $(500 \mu \mathrm{L})$ was added, and the resulting solution was stirred for 10 min to afford $0.02 \mathrm{M} \mathrm{Ni}(\mathrm{II})$ catalyst THF solution. To a solution of $1 \mathrm{a}(30.5 \mathrm{mg}, 0.100 \mathrm{mmol})$ in $\mathrm{CH}_{3} \mathrm{CN} / \mathrm{CD}_{3} \mathrm{CN}(1 / 1,900$ $\mu \mathrm{L})$ was added $100 \mu \mathrm{L}(0.002 \mathrm{mmol}, 2 \mathrm{~mol} \%)$ of the $\mathrm{Ni}(\mathrm{II})$ catalyst solution. The resulting mixture was stirred for $4 \mathrm{~h}$ at the same temperature, and the consumption of 1a was monitored by TLC. The reaction was quenched with $0.1 \mathrm{M}$ $\mathrm{AcOH} / \mathrm{THF}$ solution $(100 \mu \mathrm{L})$. After the addition of sat. $\mathrm{NaHCO}_{3}$ aqueous solution, the mixture was extracted with AcOEt, and the combined organic layers were washed with brine, dried over $\mathrm{Na}_{2} \mathrm{SO}_{4}$, and concentrated in vacuo. ${ }^{1} \mathrm{H} \mathrm{NMR}$ analysis of the crude mixture was conducted, and the product ratio of $\mathbf{2 a} / \mathbf{2} \mathbf{a}-d_{2}\left(k_{\mathrm{H}} / k_{\mathrm{D}}\right)$ was determined to be 5.37 $(84.3 \% / 15.7 \%)$.

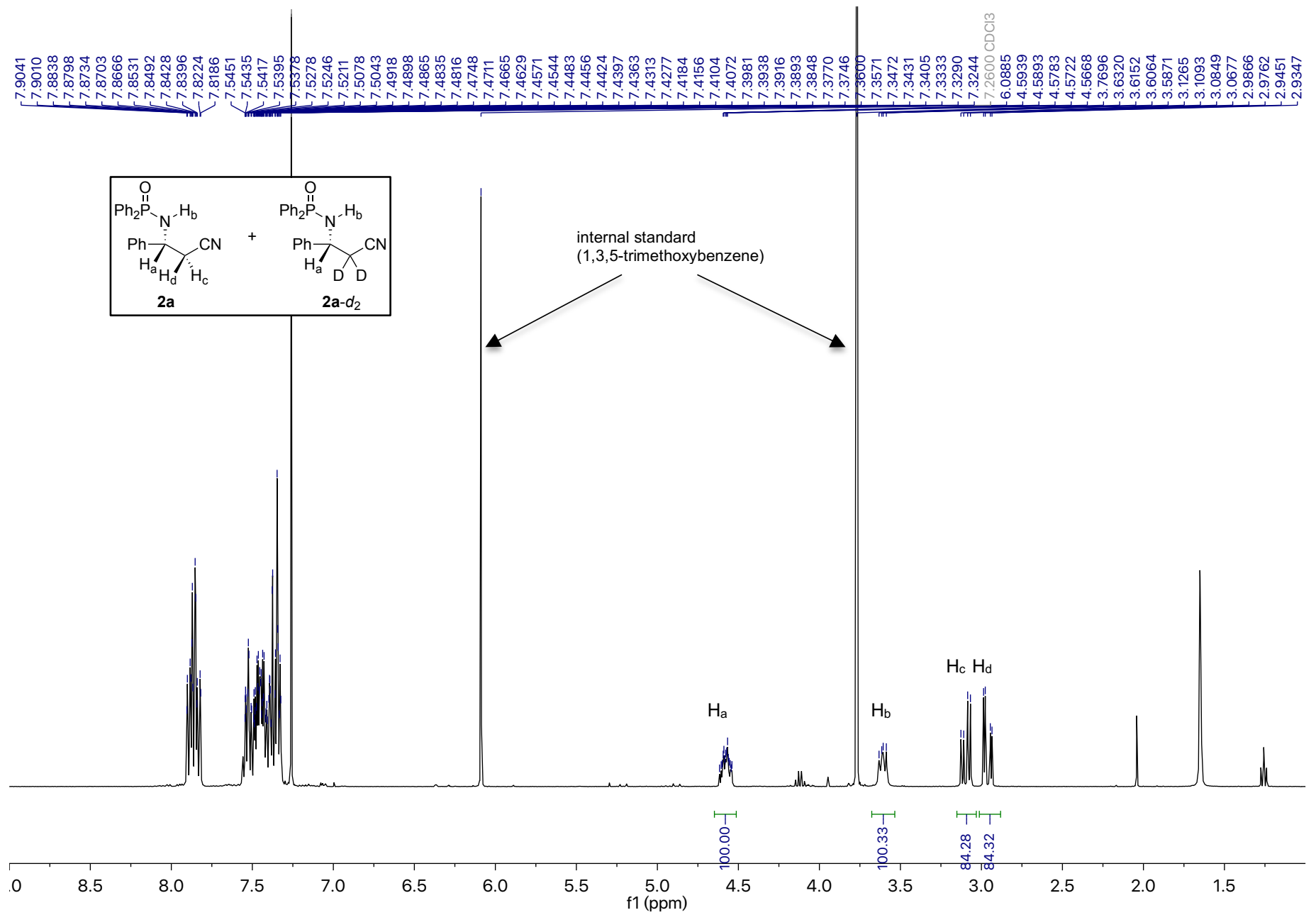




\section{6-2. Parallel reaction and initial rate kinetics}
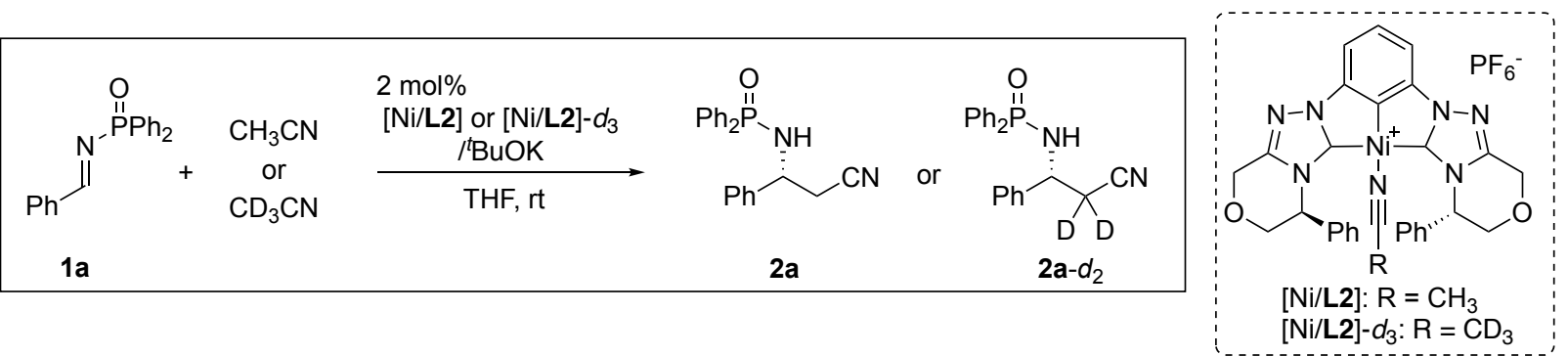

A flame-dried test tube $(20 \mathrm{~mL})$ was charged with $[\mathrm{Ni} / \mathrm{L} 2](7.2 \mathrm{mg}, 0.010 \mathrm{mmol})$ and ${ }^{t} \mathrm{BuOK}(1.1 \mathrm{mg}, 0.010 \mathrm{mmol})$ in a dry box. After the test tube was cooled down to $0{ }^{\circ} \mathrm{C}$, acetonitrile $(500 \mu \mathrm{L})$ and THF $(500 \mu \mathrm{L})$ were successively added, and the resulting solution was stirred for $10 \mathrm{~min}$ to afford $0.01 \mathrm{M} \mathrm{Ni}$ (II) catalyst solution. Several test tubes with a solution of 1a $(15.3 \mathrm{mg}, 0.050 \mathrm{mmol})$ in THF $(2.0 \mathrm{~mL})$ were prepared, and to each tube was added $100 \mu \mathrm{L}(0.001 \mathrm{mmol}, 2 \mathrm{~mol} \%)$ of the $\mathrm{Ni}$ (II) catalyst solution at room temperature. The resulting mixture was stirred for specific time and was quenched by the addition of $0.1 \mathrm{M} \mathrm{AcOH} / \mathrm{THF}$ solution $(100 \mu \mathrm{L})$. After removal of solvent, substrate/product ratio was determined by ${ }^{1} \mathrm{H}$ NMR analysis. The experiment using $\mathrm{CD}_{3} \mathrm{CN}$ and [Ni/L2]- $d_{3}$, which could be prepared by repetitive addition and evaporation of $\mathrm{CD}_{3} \mathrm{CN}$ for three times, was performed followed by the same procedure. The obtained yield-time relationship was plotted, and $k_{\mathrm{H}} / k_{\mathrm{D}}$ value was determined to be $3.00(=24.8 / 8.26)$.

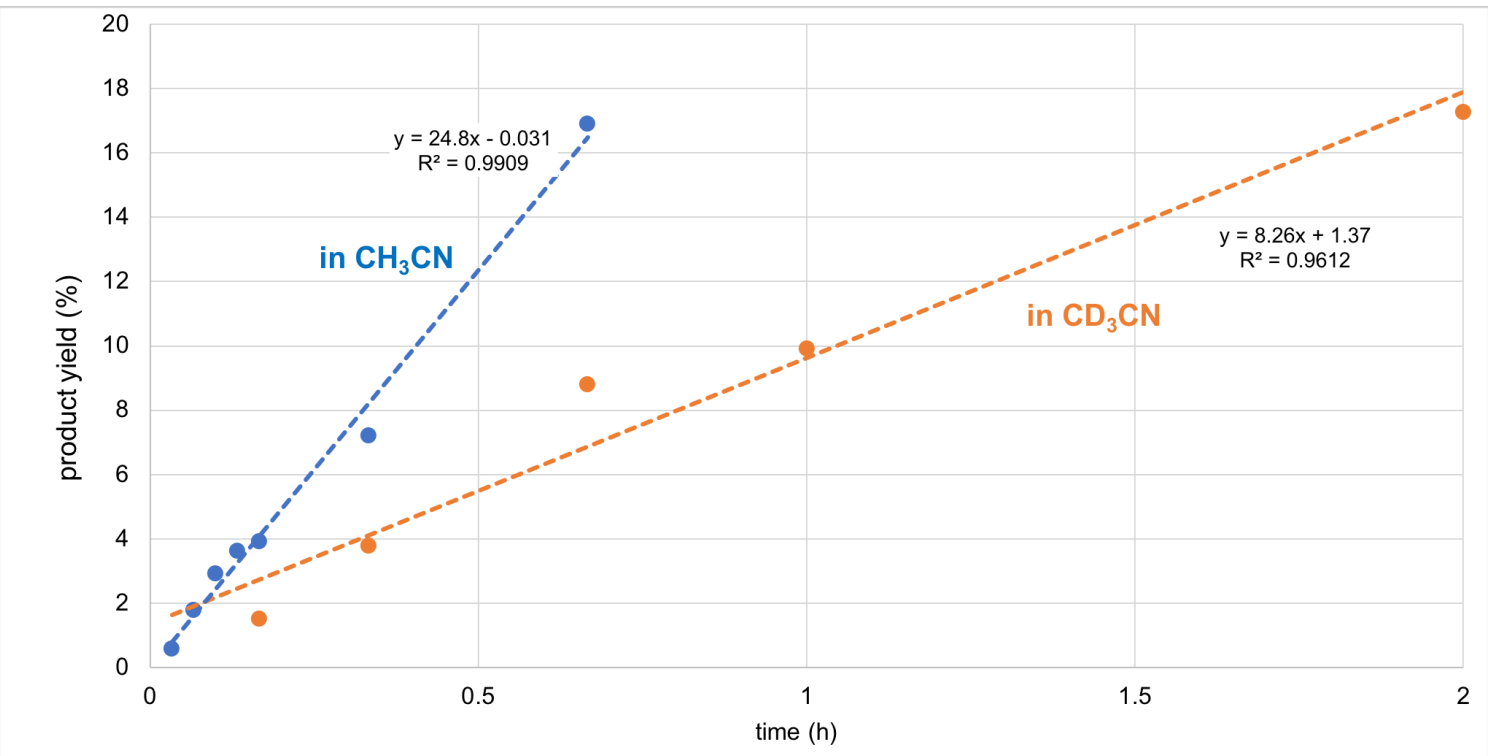




\section{References}

1. Fulmer, G. R.; Miller, A. J. M.; Sherden, N. H.; Gottlieb, H. E.; Nudelman, A.; Stoltz, B. M.; Bercaw, J. E.; Goldberg, K. I. Organometallics 2010, 29, 2176.

2. Crampton, R. H.; El Hajjaji, S.; Fox, M. E.; Woodward, S. Tetrahedron: Asymmetry 2009, $20,2497$.

3. a) Ma, Y.; Wei, S.; Wu, J.; Yang, F.; Liu, B.; Lan, J.; Yang, S.; You, J. Adv. Synth. Catal. 2008, 350, 2645. b) Wei, S.; Li, X.; Yang, Z.; Lan, J.; Gao, G.; Xue, Y.; You, J. Chem. Sci. 2012, 3, 359.

4. Rajca, A.; Olankitwanit, A.; Rajca, R. J. Am. Chem. Soc. 2011, 133, 4750.

5. Chen, Z.; Huo, Y.; An, P.; Wang, X.; Song, C.; Ma, Y. Org. Chem. Front. 2016, 3, 1725.

6. Kawato, Y.; Kumagai, N.; Shibasaki, M. Chem. Commun. 2013, 49, 11227.

7. Dolomanov, O.V.; Bourhis, L. J.; Gildea, R. J.; Howard, J. A. K.; Puschmann, H. J. Appl. Cryst. 2009, 42, 339. 


\section{8. ${ }^{1} \mathrm{H}$ and ${ }^{13} \mathrm{C}$ NMR Spectra}

(5S,5'S)-2,2'-(1,3-Phenylene)bis(5-(tert-butyl)-5,6-dihydro-8H-[1,2,4]triazolo[3,4-c][1,4]oxazin-2-ium) dichloride (S1c): 1 H NMR $\left(400 \mathrm{MHz}, \mathrm{CD}_{3} \mathrm{CN}\right)$

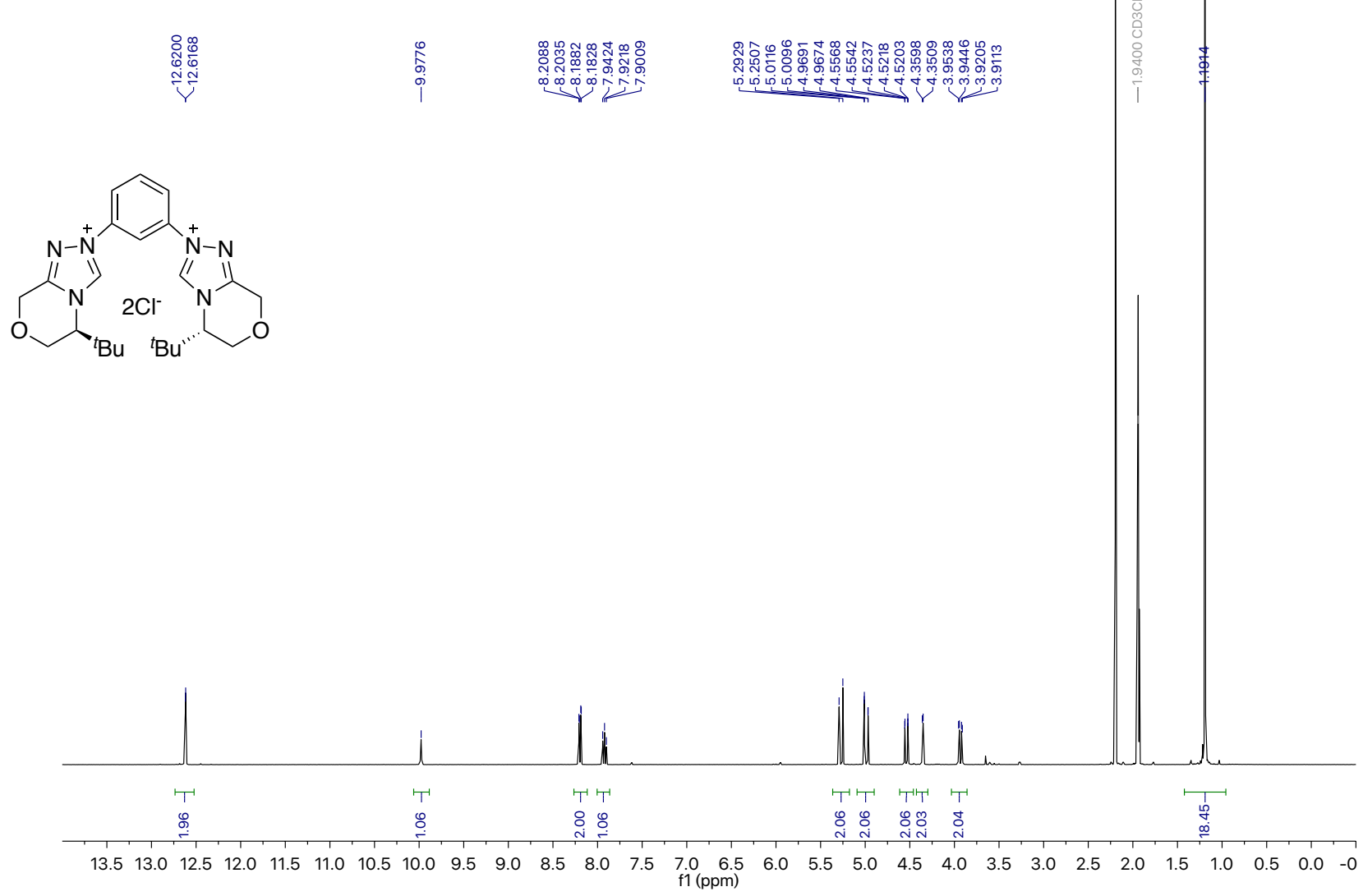

${ }^{13} \mathrm{C}$ NMR $\left(100 \mathrm{MHz}, \mathrm{CD}_{3} \mathrm{CN}\right)$<smiles></smiles>

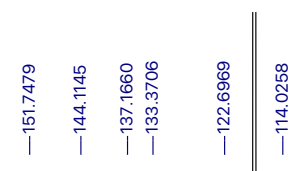

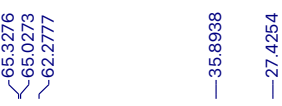$$
\begin{array}{llllllllllllllllllllllll}
220 & 210 & 200 & 190 & 180 & 170 & 160 & 150 & 140 & 130 & 120 & 110 & 100 & 90 & 80 & 70 & 60 & 50 & 40 & 30 & 20 & 10 & 0 & -1
\end{array}
$$ 
Ni(II) pincer complex (S4a):

${ }^{1}$ H NMR $\left(400 \mathrm{MHz}, \mathrm{CDCl}_{3}\right)$

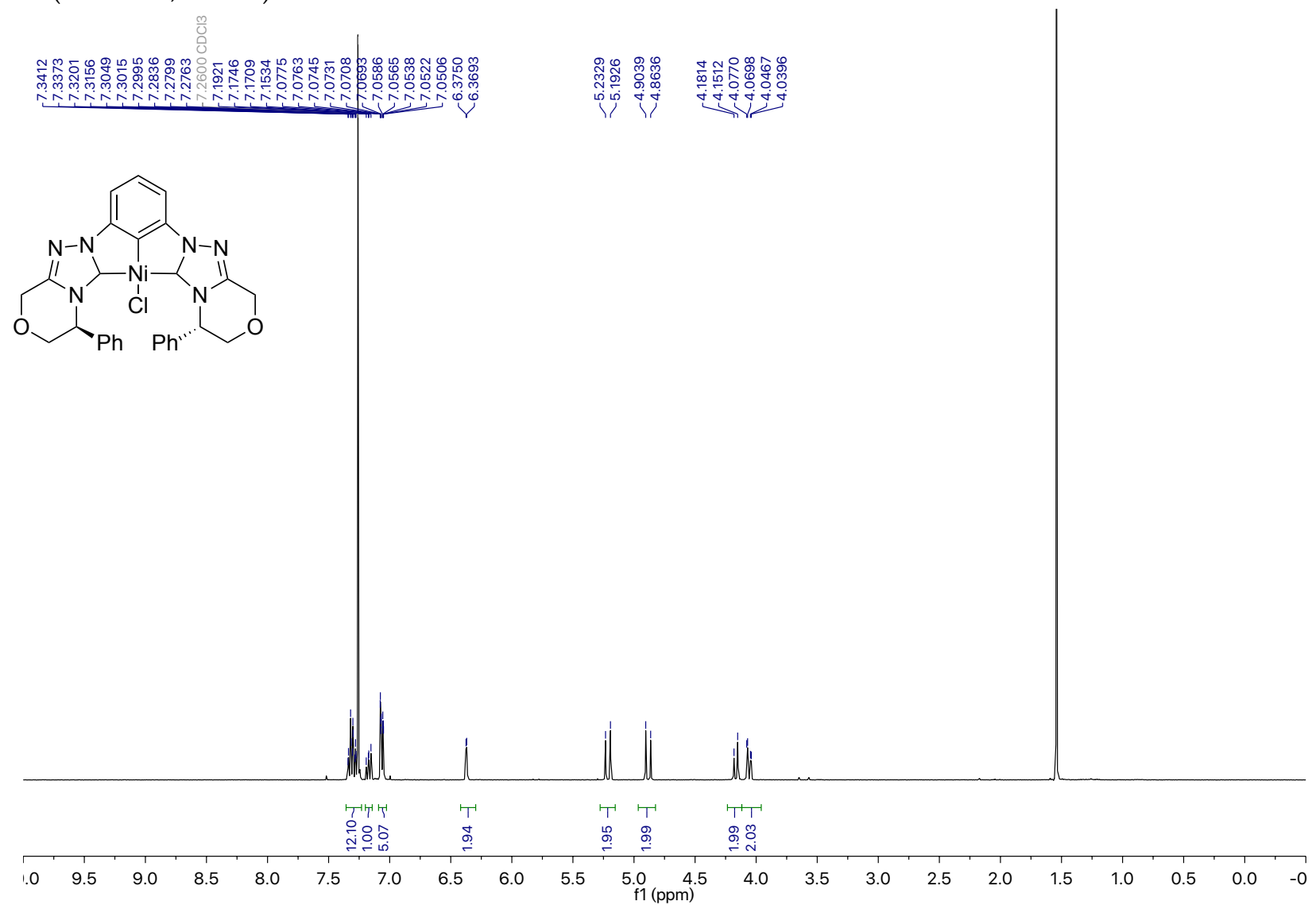

${ }^{13} \mathrm{C}$ NMR (100 MHz, $\left.\mathrm{CD}_{2} \mathrm{Cl}_{2}\right)$

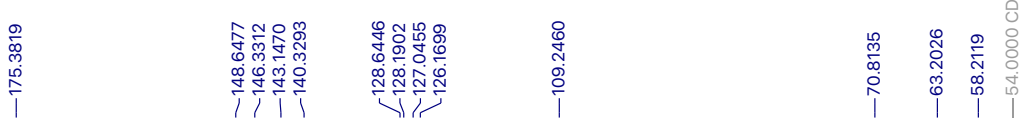
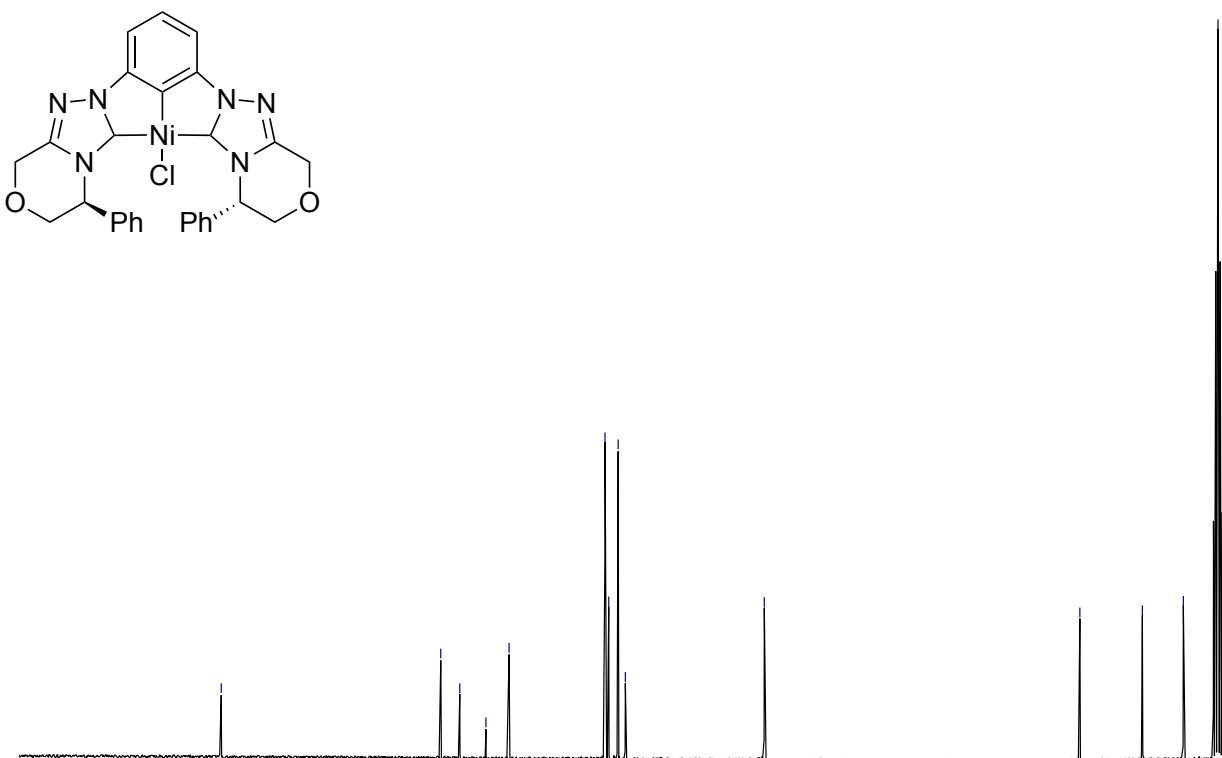

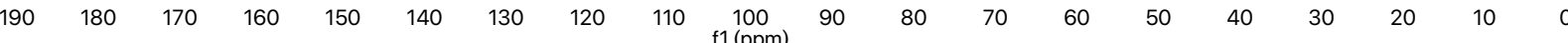


cationic $\mathrm{Ni}(\mathrm{II})$ pincer complex (Ni/L2):

${ }^{1} \mathbf{H}$ NMR $\left(400 \mathrm{MHz}, \mathrm{CD}_{2} \mathrm{Cl}_{2}\right)$

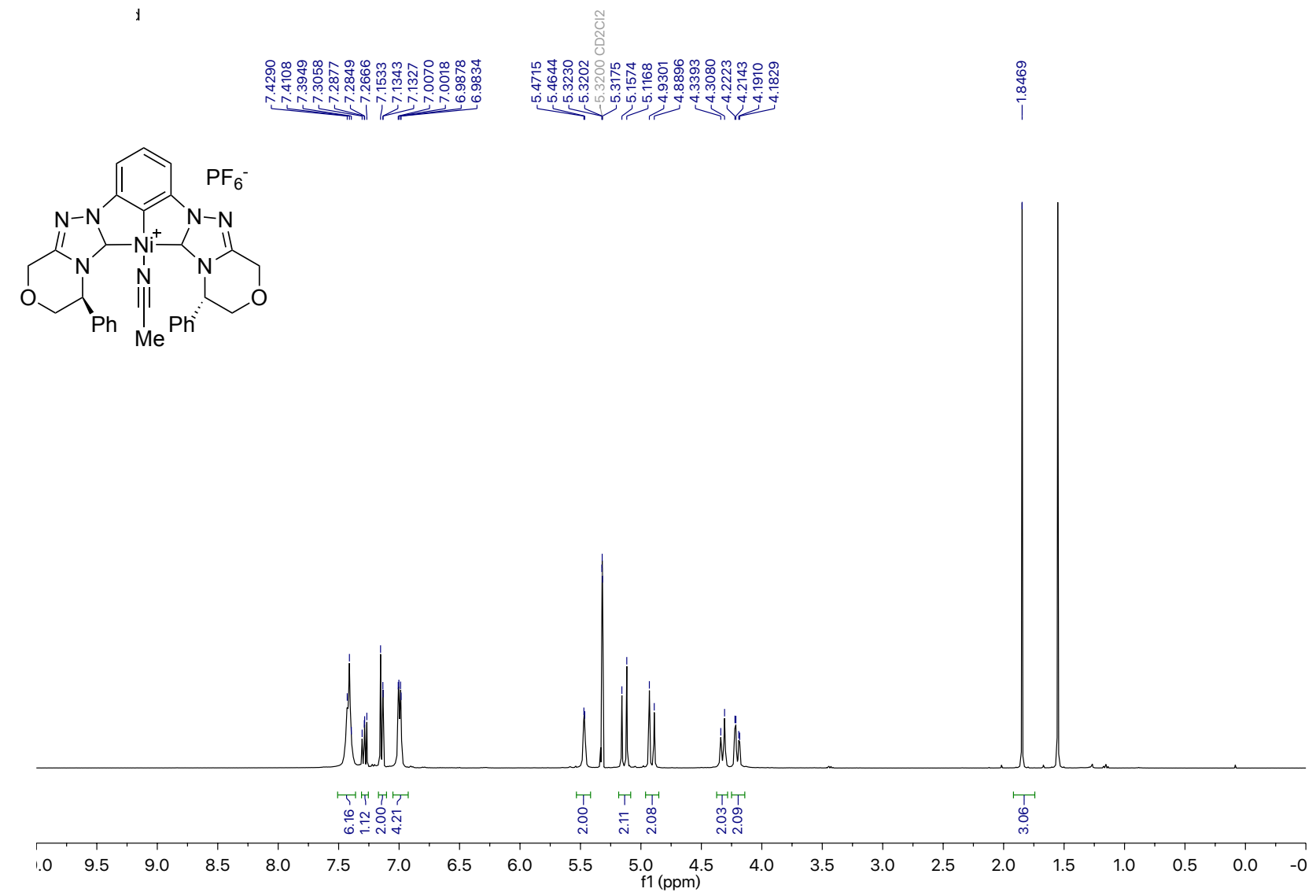

${ }^{13} \mathrm{C}$ NMR (100 MHz, $\left.\mathrm{CD}_{2} \mathrm{Cl}_{2}\right)$

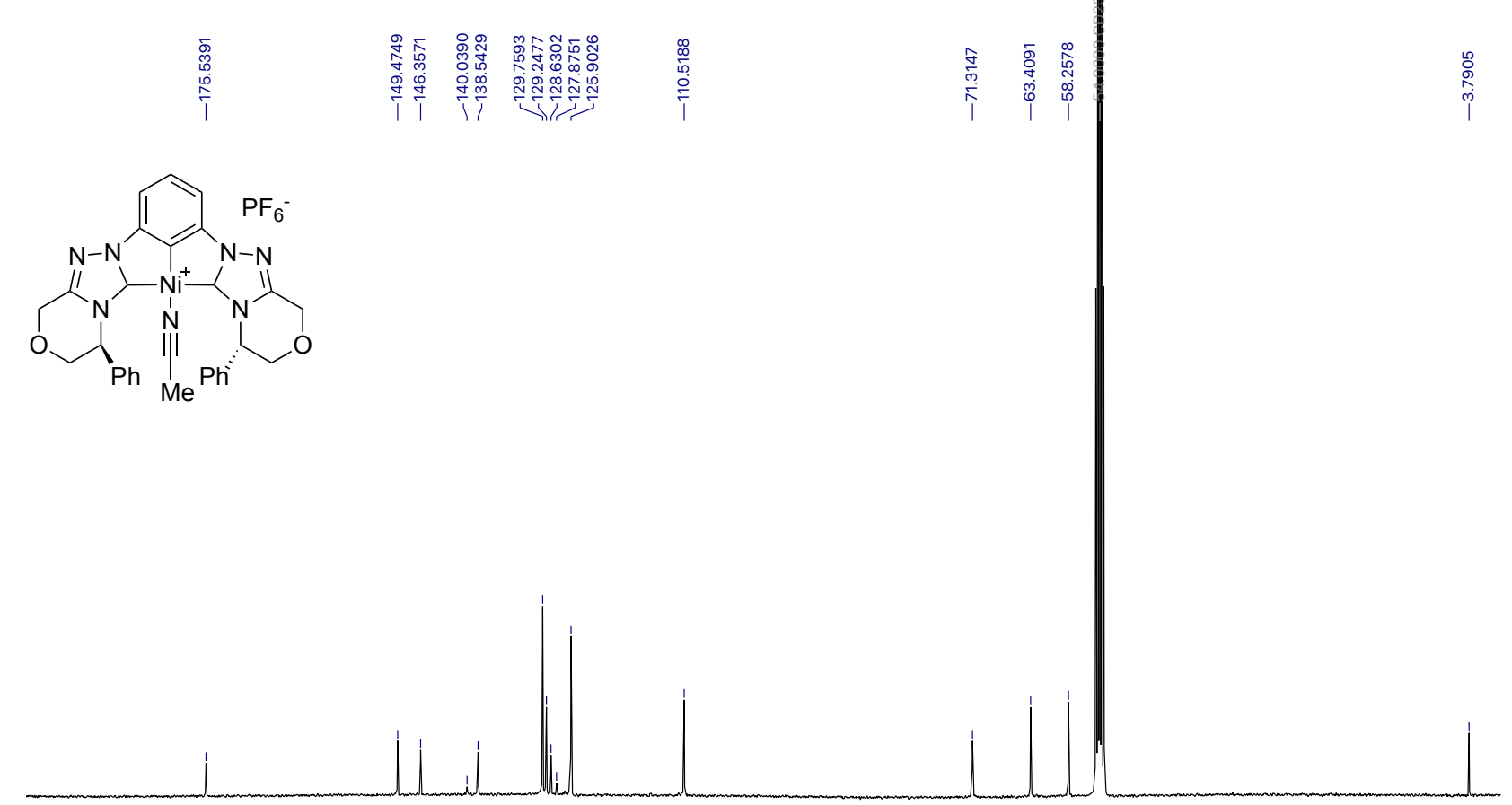

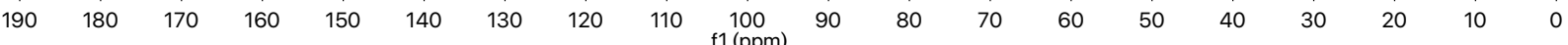


Ni(II) pincer complex (S4b):

${ }_{1}^{1} \mathbf{H}$ NMR (400 MHz, $\mathrm{CDCl}_{3}$ )

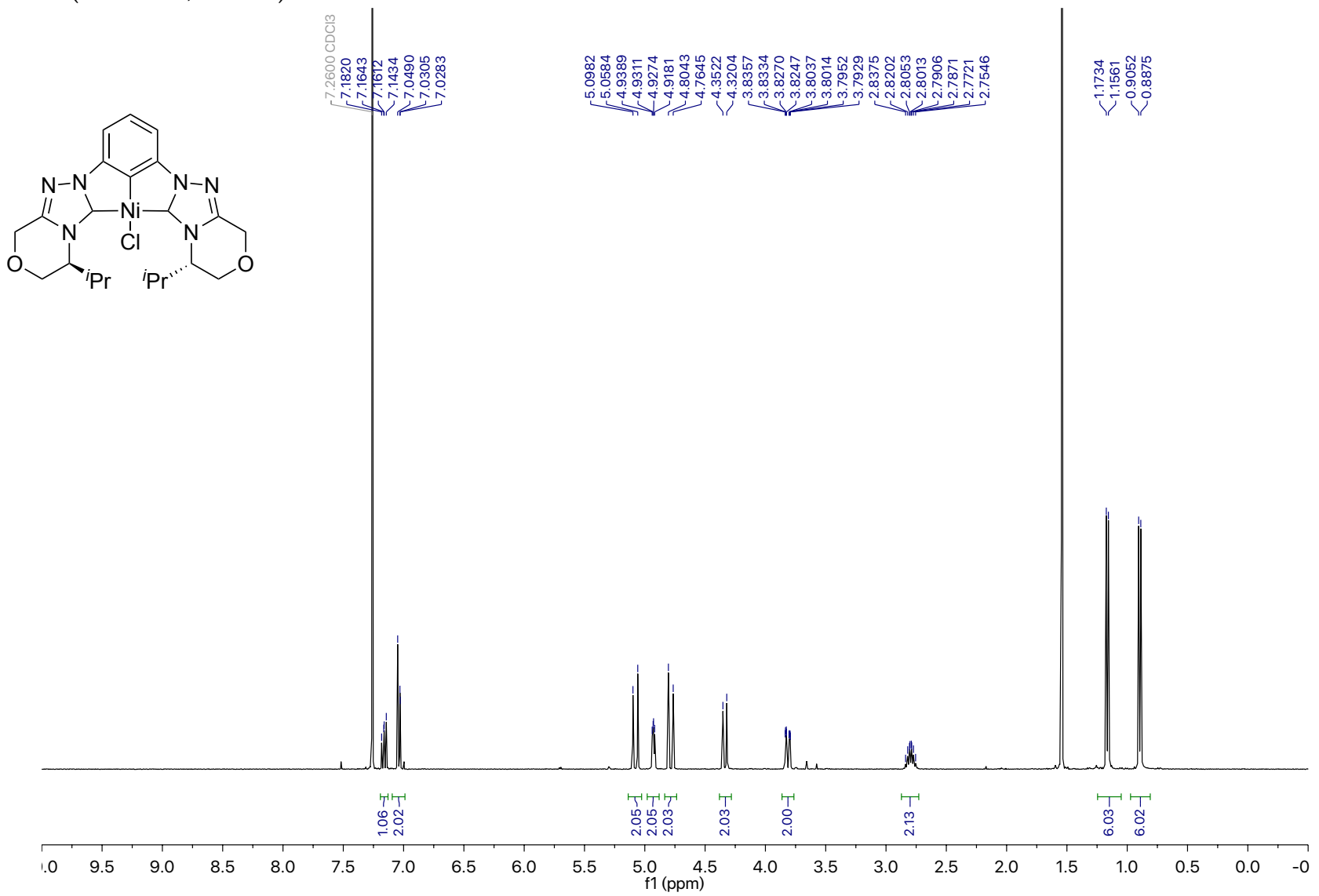

${ }^{13} \mathrm{C}$ NMR (100 MHz, $\left.\mathrm{CDCl}_{3}\right)$

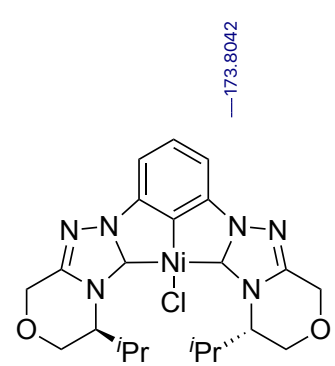


Ni(II) pincer complex (S4c):

${ }_{1}^{1} \mathbf{H}$ NMR (400 MHz, $\mathrm{CDCl}_{3}$ )

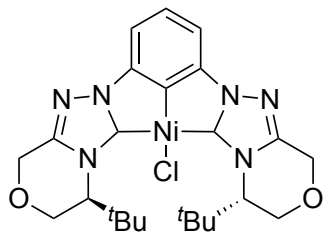
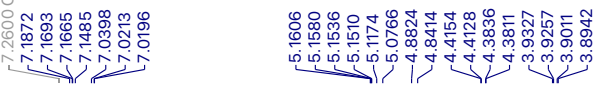

rinin

政

${ }^{t} \mathrm{Bu}$
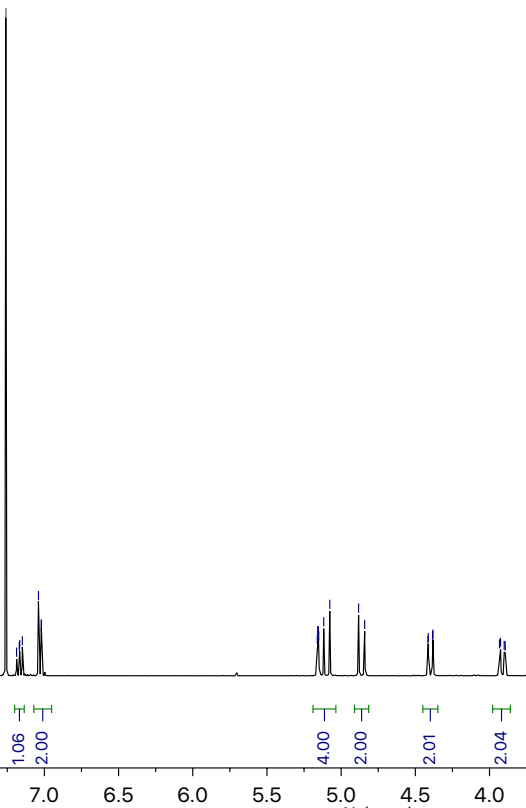

$\begin{array}{lll}T & T \\ T & T & D^{2}\end{array}$

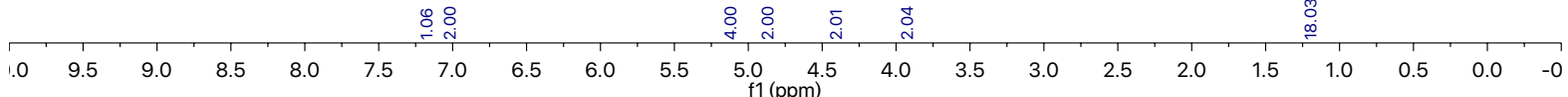

${ }^{13} \mathrm{C}$ NMR (100 MHz, $\left.\mathrm{CDCl}_{3}\right)$
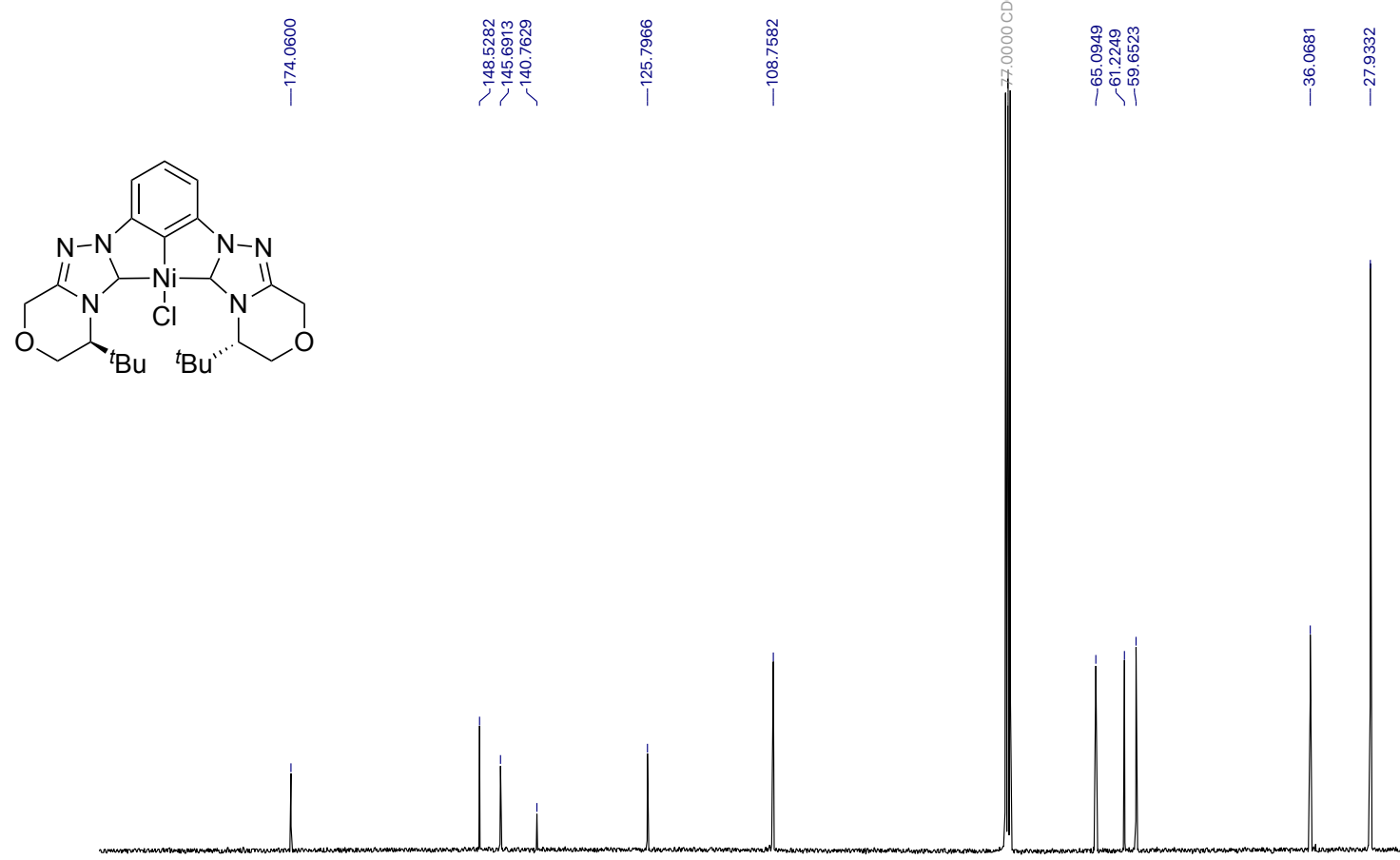

$\begin{array}{llllllllll}190 & 180 & 170 & 160 & 150 & 140 & 130 & 120 & 110 & \begin{array}{l}100 \\ f 1(p p m)\end{array}\end{array}$ 
Ni(II) pincer complex (S4d):

${ }^{1} \mathbf{H}$ NMR $\left(400 \mathrm{MHz}, \mathrm{CDCl}_{3}\right)$

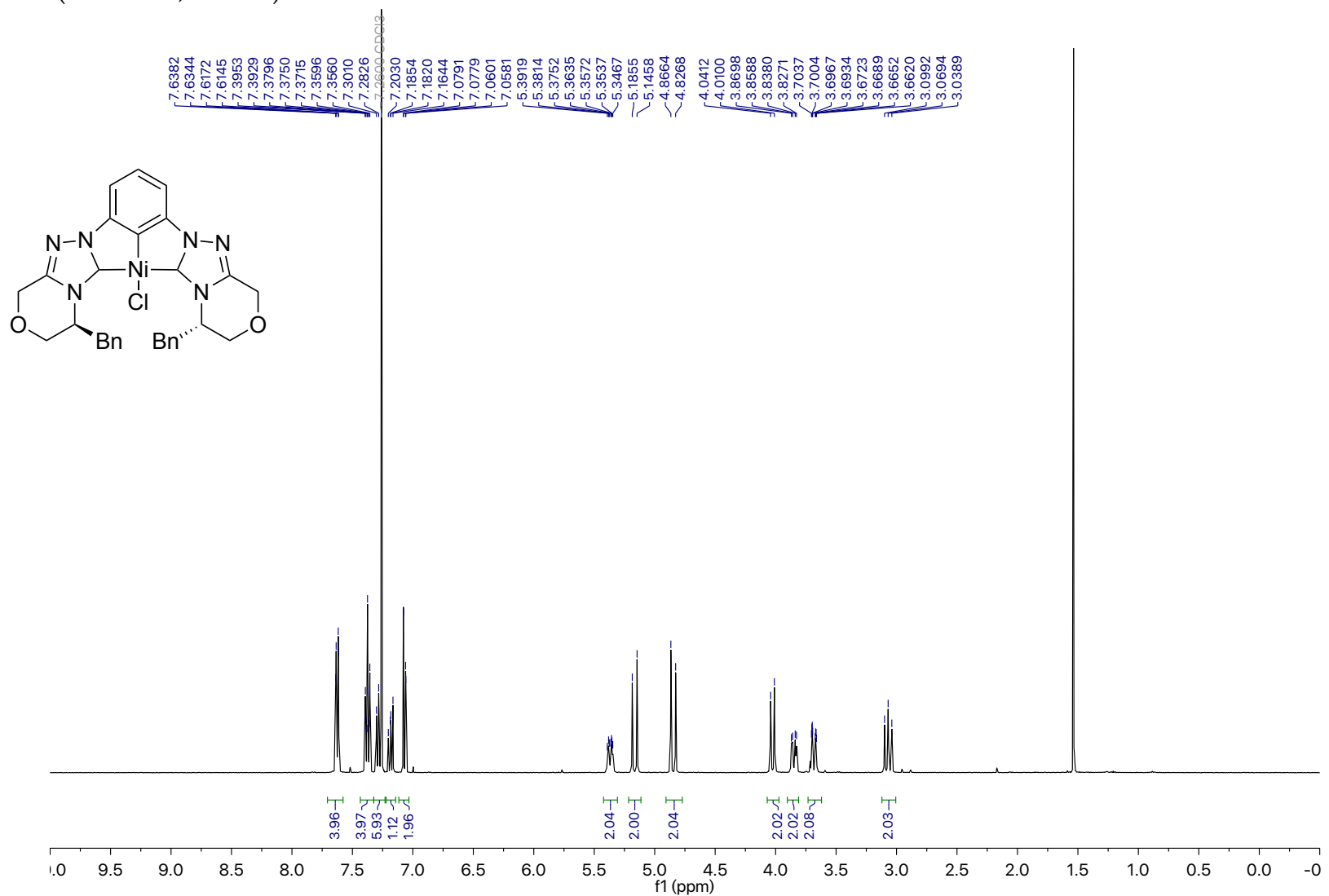

${ }^{13} \mathrm{C} \mathrm{NMR}\left(100 \mathrm{MHz}, \mathrm{CDCl}_{3}\right)$

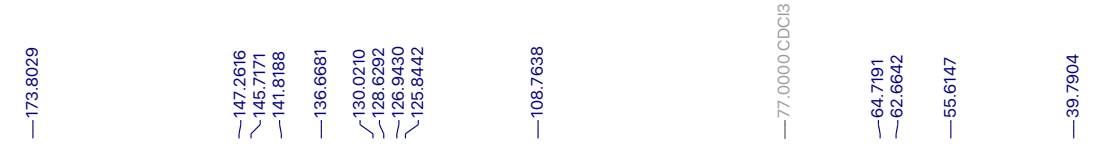

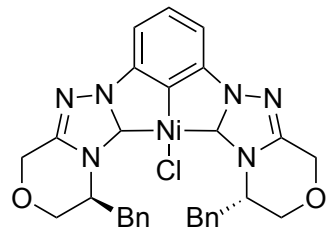

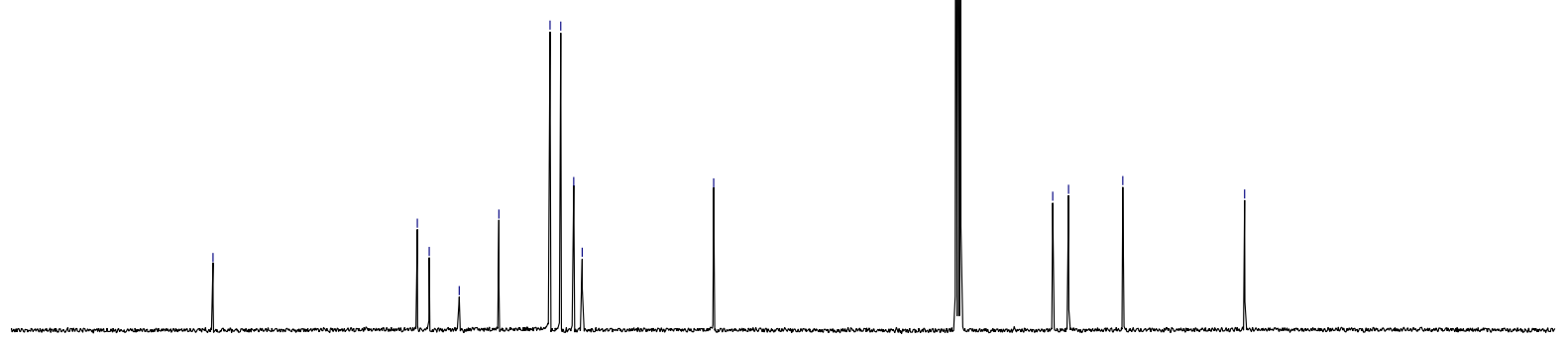

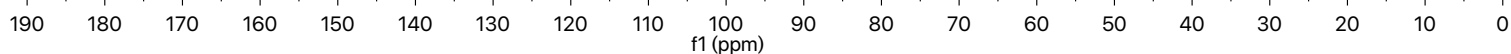


(R)-N-(2-Cyano-1-phenylethyl)-P,P-diphenylphosphinic amide (2a):

${ }^{1} \mathbf{H}$ NMR (400 MHz, $\left.\mathrm{CDCl}_{3}\right)$
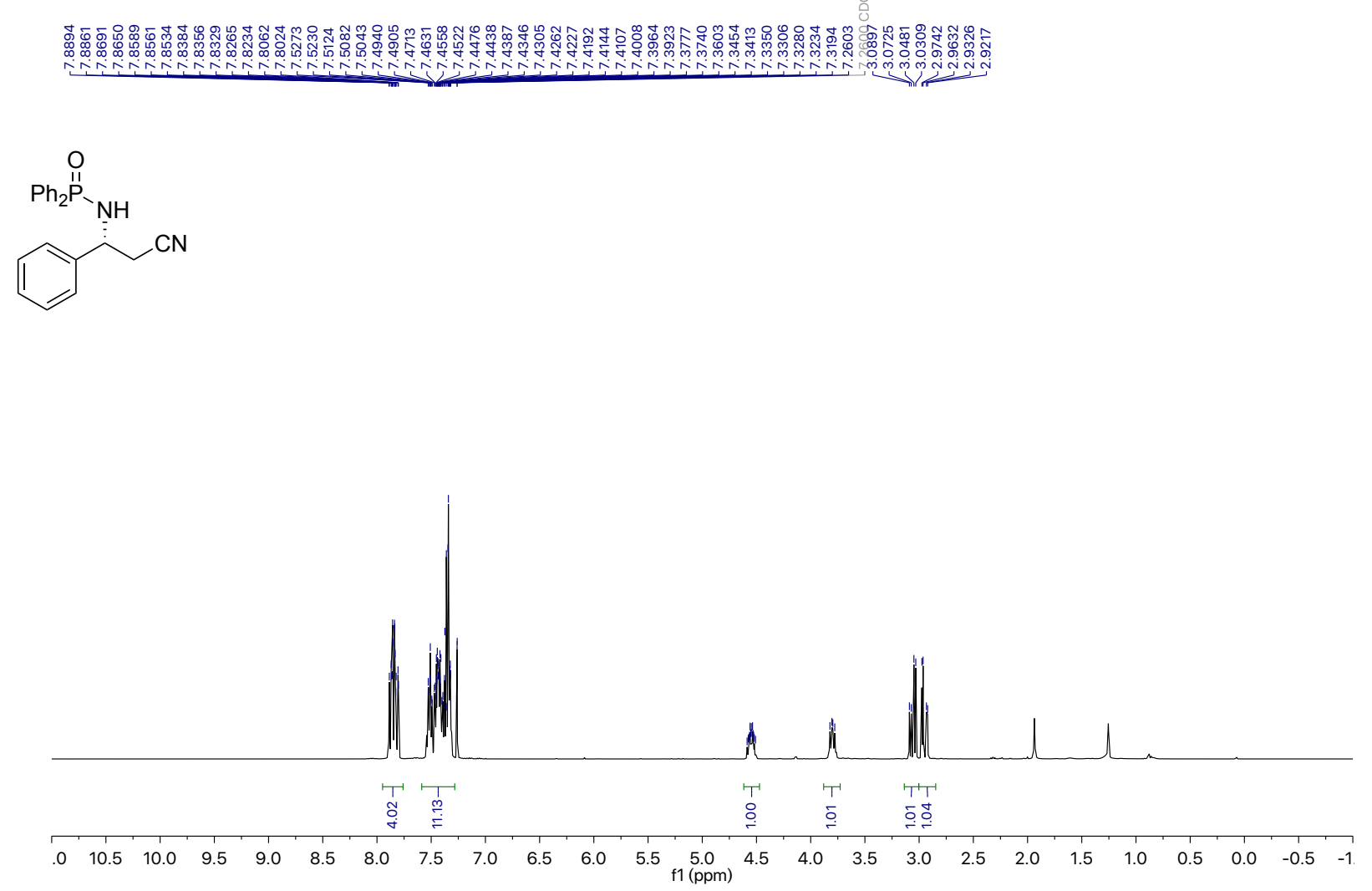

${ }^{13} \mathrm{C}$ NMR $\left(100 \mathrm{MHz}, \mathrm{CDCl}_{3}\right)$
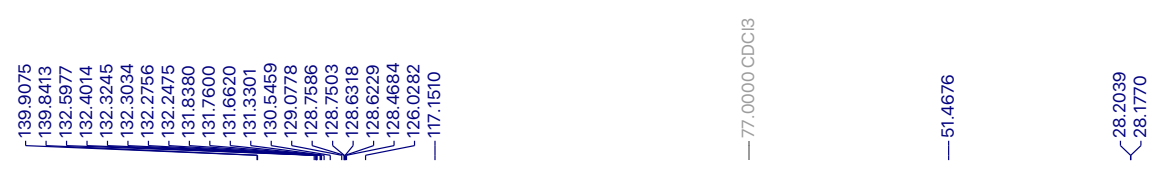<smiles>N#CCC(NP(=O)(O)c1ccccc1)c1ccccc1</smiles>

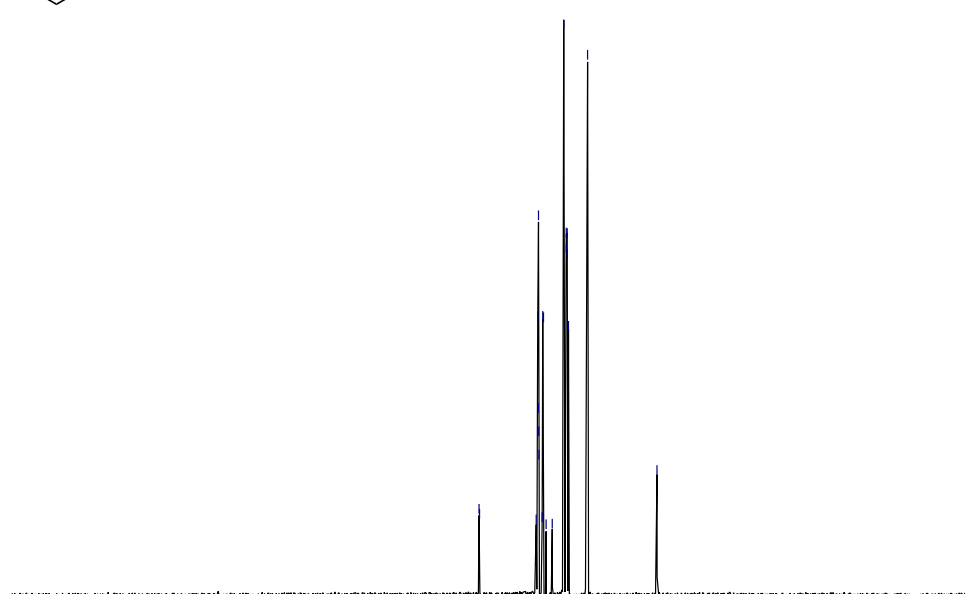

$\begin{array}{llllllllll}190 & 180 & 170 & 160 & 150 & 140 & 130 & 120 & 110 & 100 \\ \mathrm{f} 1(\mathrm{ppm})\end{array}$ 
(R)-N-(2-Cyano-1-(o-tolyl)ethyl)-P,P-diphenylphosphinic amide (2b):

${ }^{1}$ H NMR $\left(400 \mathrm{MHz}, \mathrm{CDCl}_{3}\right)$

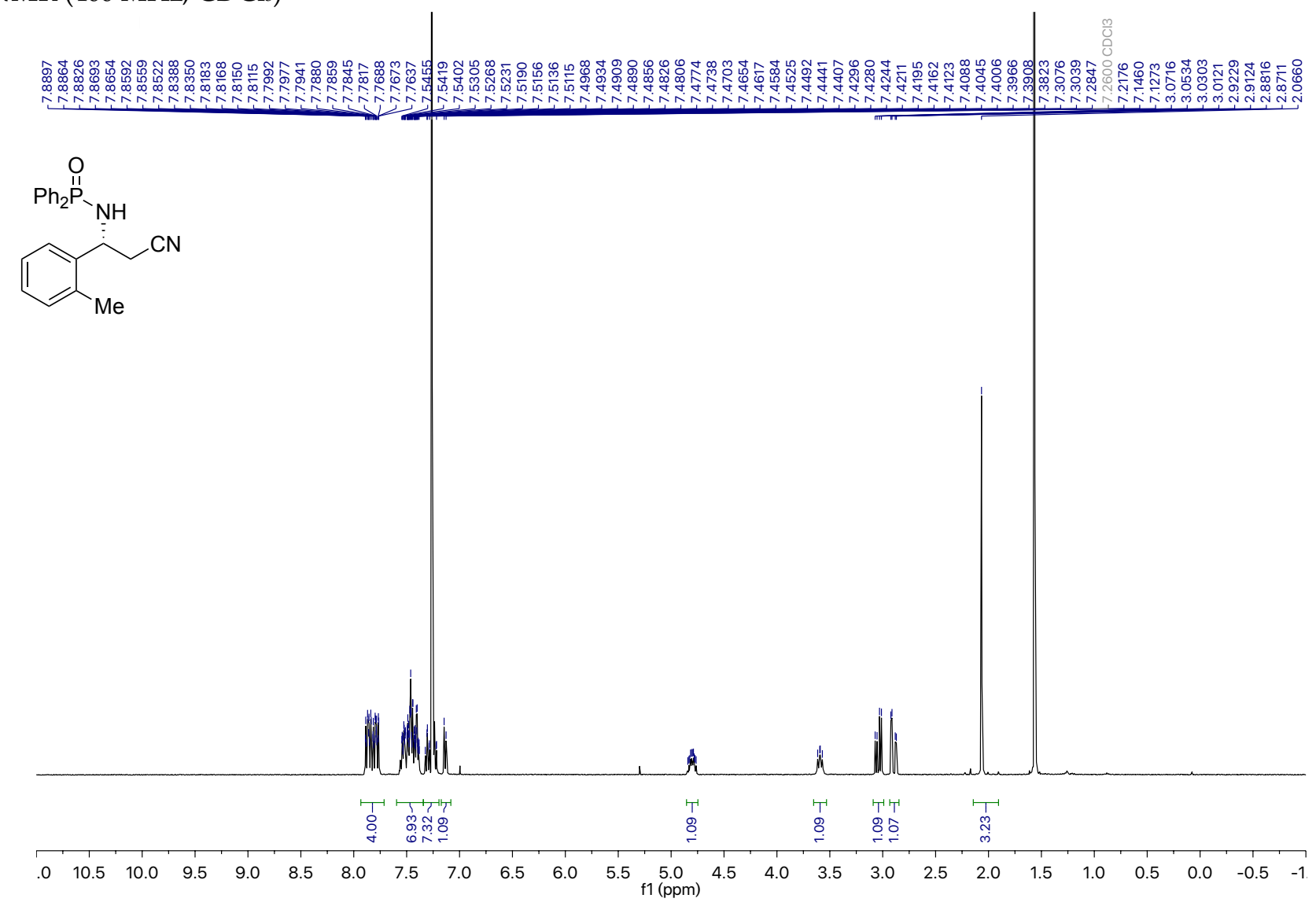

${ }^{13} \mathrm{C}$ NMR $\left(100 \mathrm{MHz}, \mathrm{CDCl}_{3}\right)$

$x$

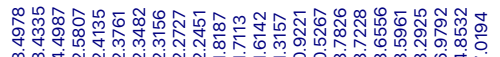

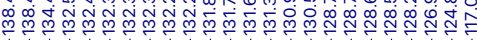<smiles>Cc1ccccc1C(CC#N)NP=O</smiles>

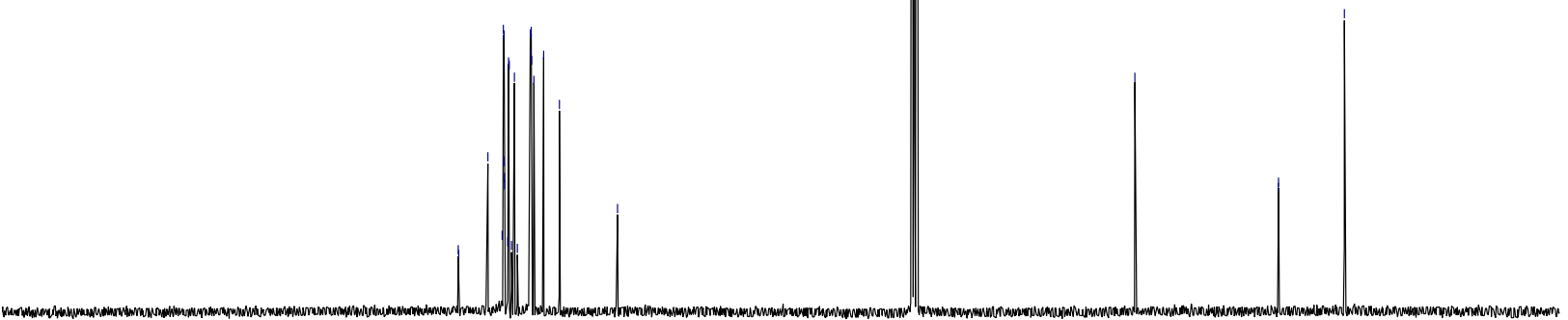

$\begin{array}{lllllllllllllllllllll}190 & 180 & 170 & 160 & 150 & 140 & 130 & 120 & 110 & 100 & 90 & 80 & 70 & 60 & 50 & 40 & 30 & 20 & 10 & 0 & -1\end{array}$ 
(R)-N-(2-Cyano-1-(naphthalen-2-yl)ethyl)-P,P-diphenylphosphinic amide (2c):

${ }^{1}$ H NMR $\left(400 \mathrm{MHz}, \mathrm{CDCl}_{3}\right)$

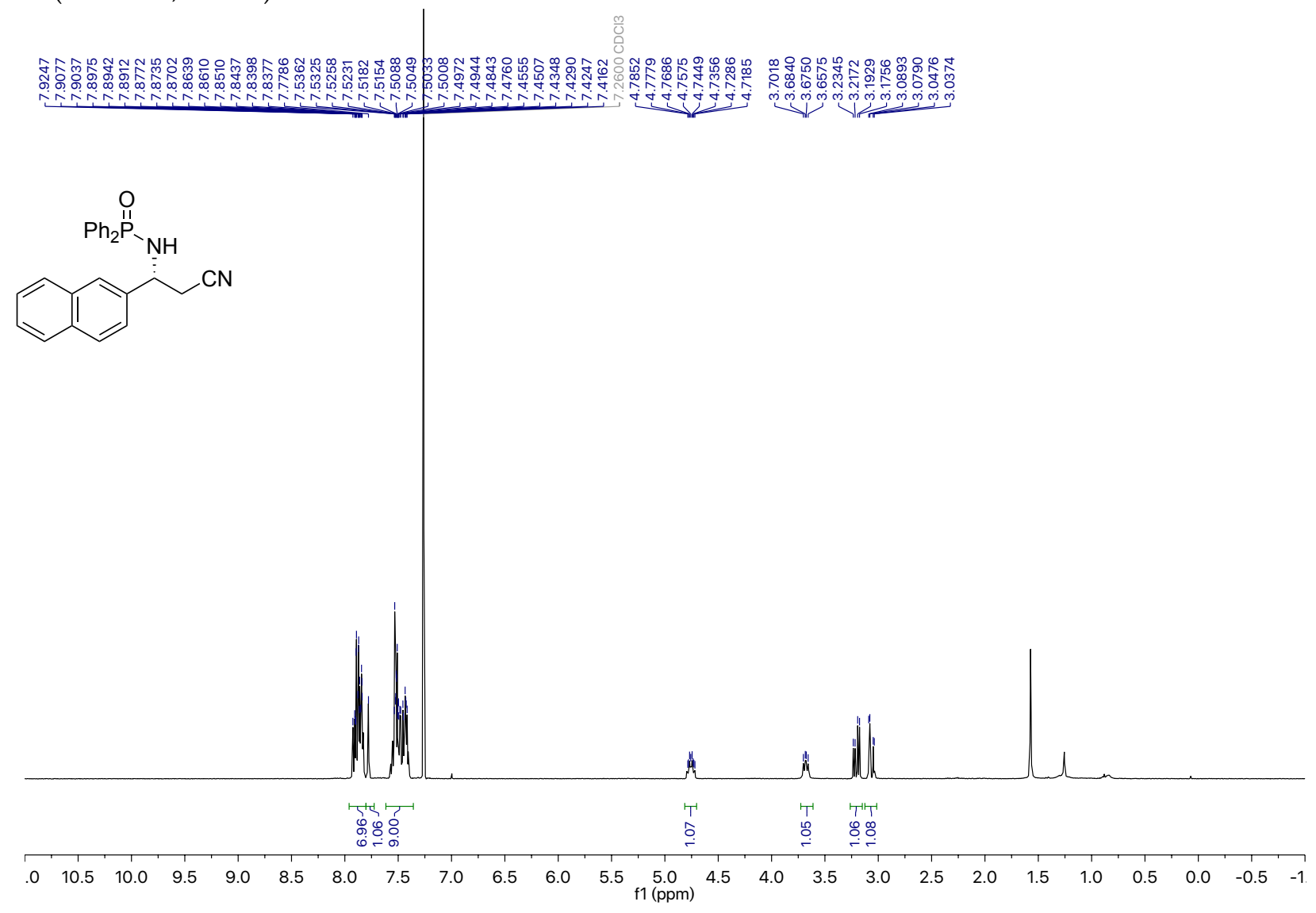

${ }^{13} \mathrm{C}$ NMR $\left(100 \mathrm{MHz}, \mathrm{CDCl}_{3}\right)$

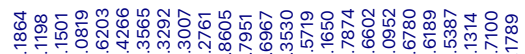

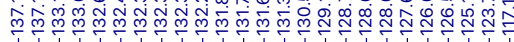

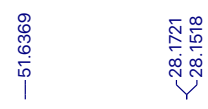<smiles>N#CCCc1ccc2ccccc2c1NPOPNc1ccccc1</smiles>

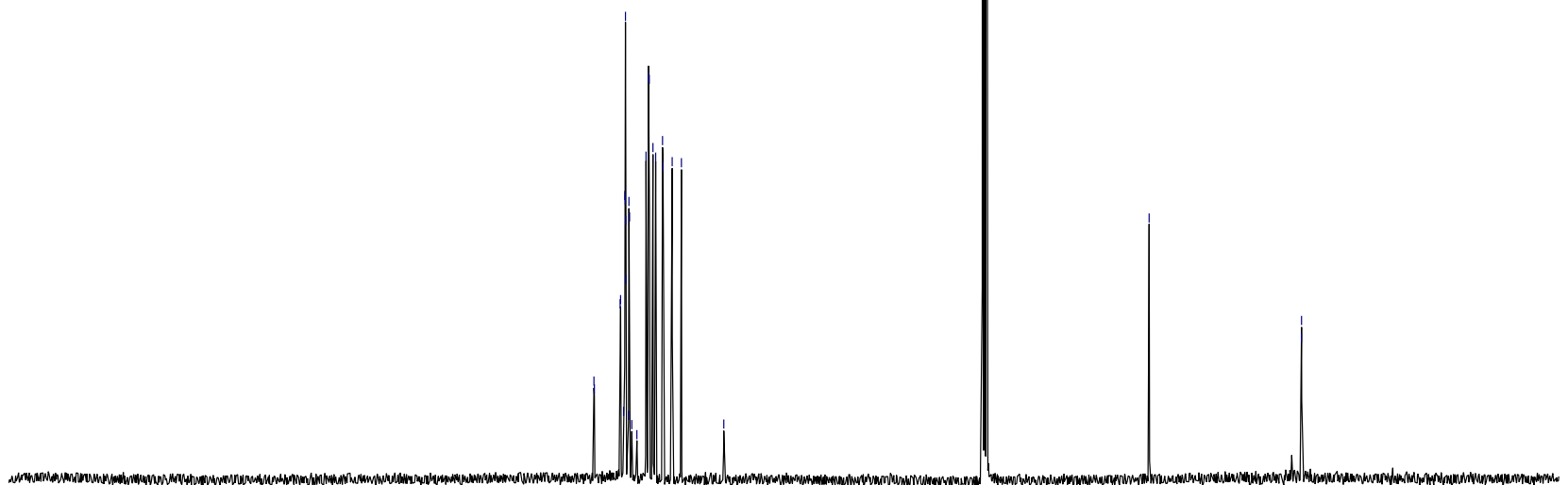

$\begin{array}{lllllllllllllllllllllllll}220 & 210 & 200 & 190 & 180 & 170 & 160 & 150 & 140 & 130 & 120 & 110 & 100 & 90 & 80 & 70 & 60 & 50 & 40 & 30 & 20 & 10 & 0 & -1 C\end{array}$ 
(R)-N-(2-Cyano-1-(naphthalen-1-yl)ethyl)-P,P-diphenylphosphinic amide (2d):

${ }_{1}^{1} \mathrm{H}$ NMR $\left(400 \mathrm{MHz}, \mathrm{CDCl}_{3}\right)$

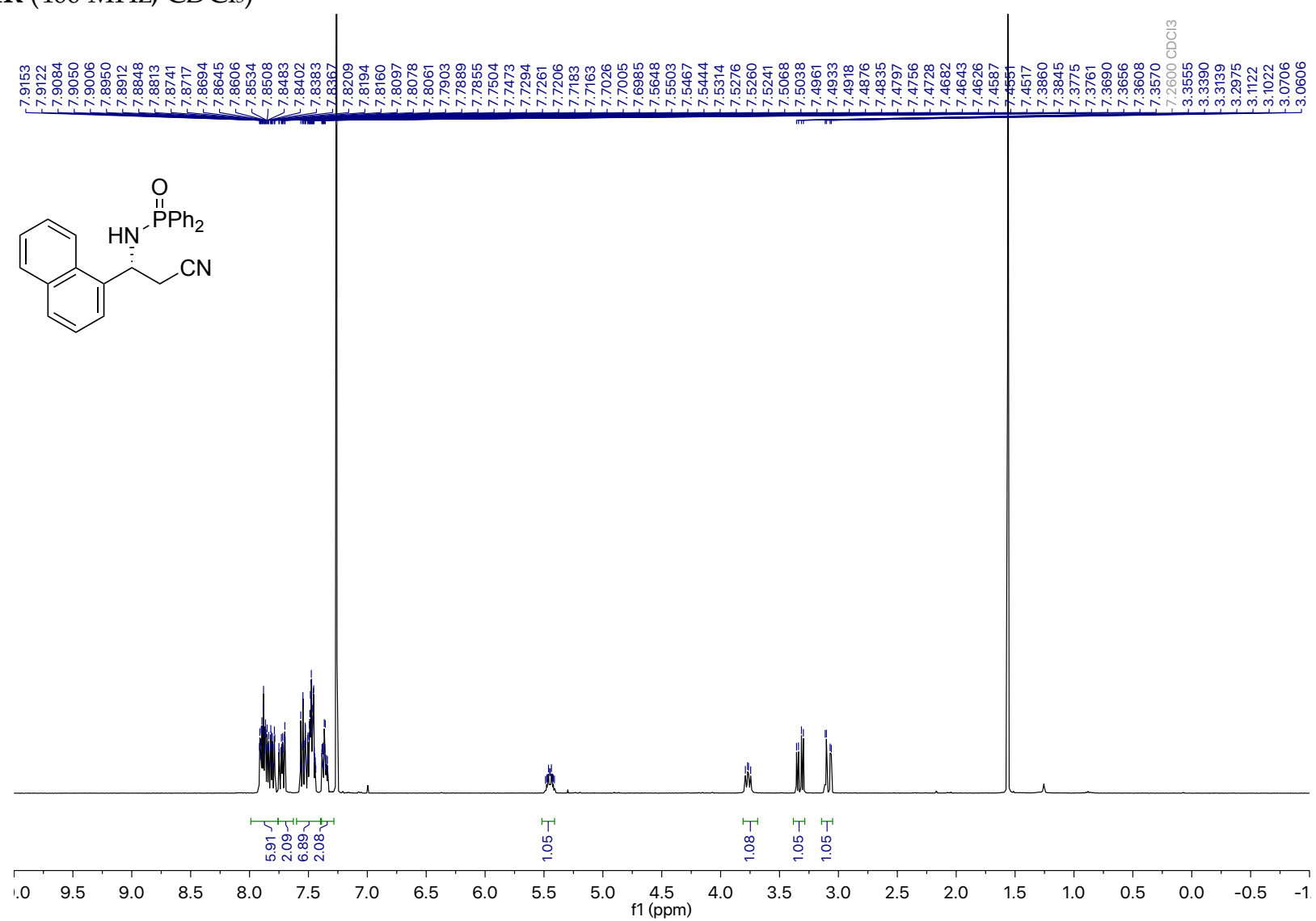

${ }^{13} \mathrm{C}$ NMR $\left(100 \mathrm{MHz}, \mathrm{CDCl}_{3}\right)$<smiles>N#CCCc1cccc2ccccc12</smiles>

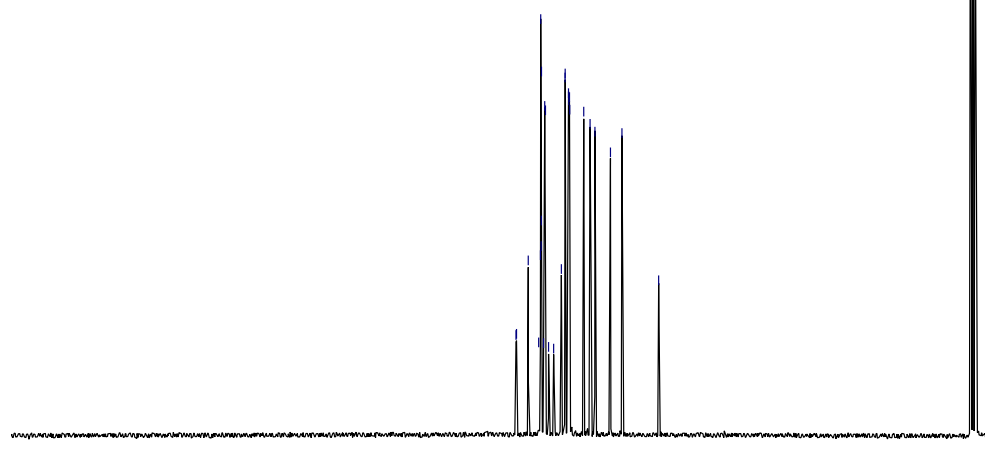

$\begin{array}{llllllllll}190 & 180 & 170 & 160 & 150 & 140 & 130 & 120 & 110 & \begin{array}{c}100 \\ \mathrm{f} 1(\mathrm{ppm})\end{array}\end{array}$ 
(R)- $N$-(2-Cyano-1-(4-methoxyphenyl)ethyl)-P,P-diphenylphosphinic amide (2e):

${ }^{1}$ H NMR $\left(400 \mathrm{MHz}, \mathrm{CDCl}_{3}\right)$

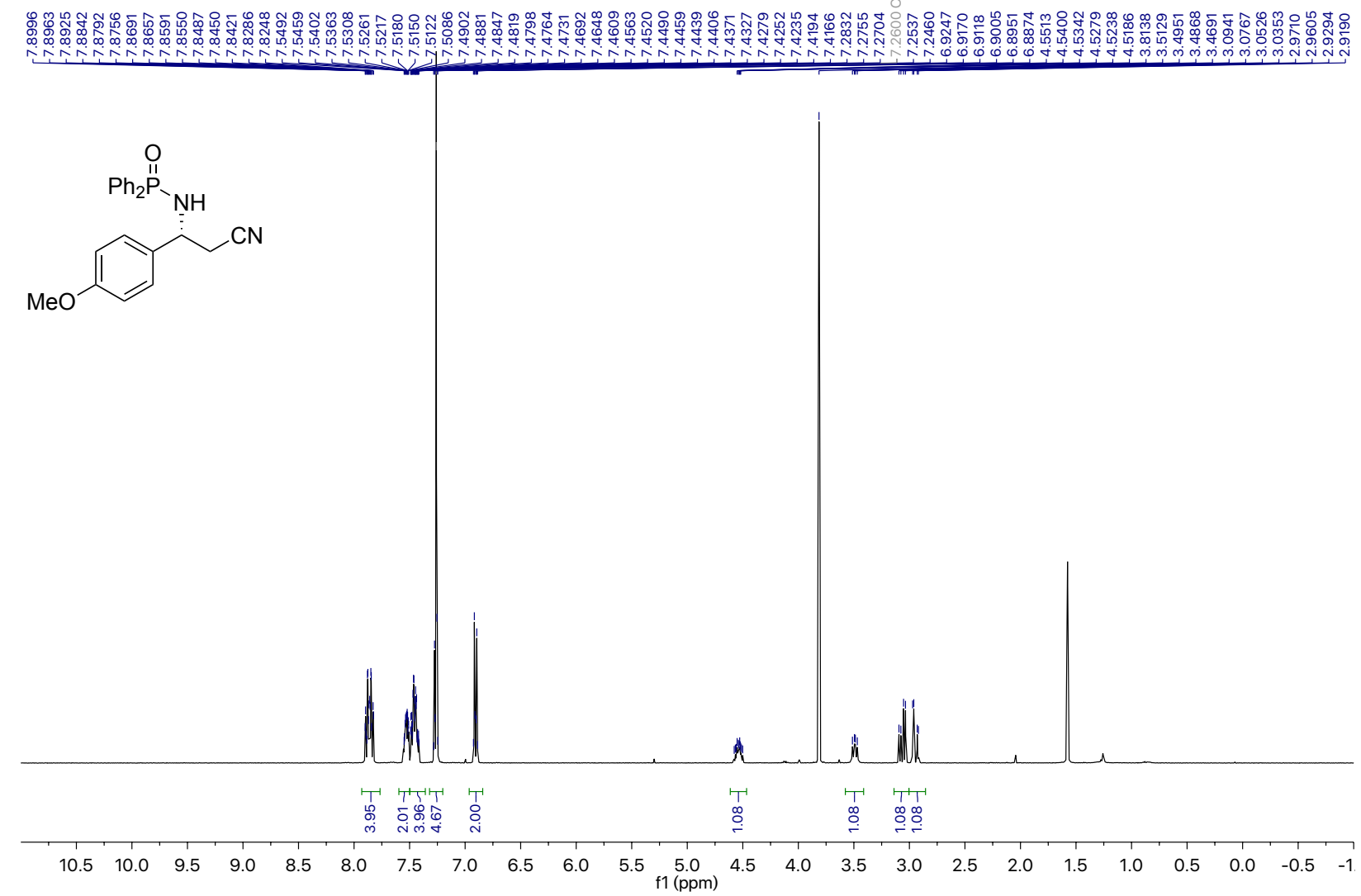

${ }^{13} \mathrm{C}$ NMR $\left(100 \mathrm{MHz}, \mathrm{CDCl}_{3}\right)$
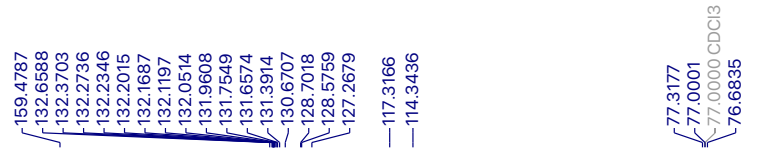

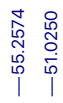

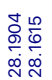<smiles>COc1ccc(CCC#N)cc1</smiles>

$\mathrm{MeO}$
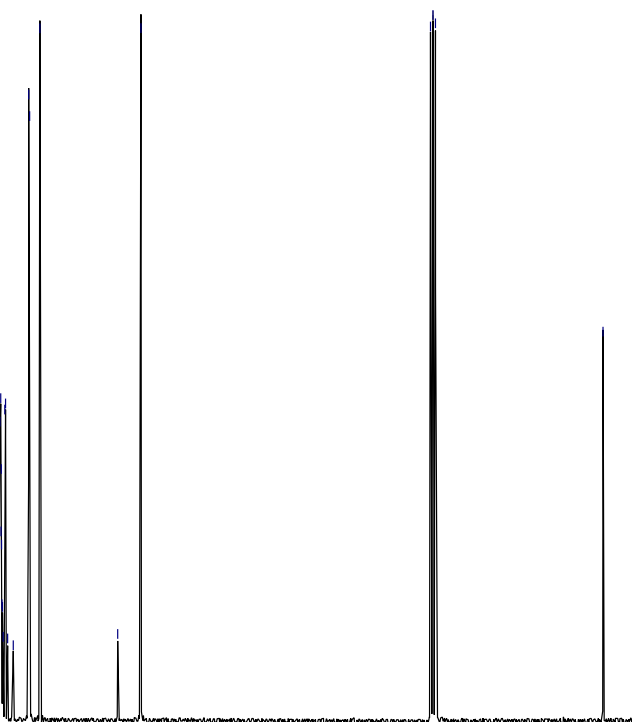

$\begin{array}{llllllllll}190 & 180 & 170 & 160 & 150 & 140 & 130 & 120 & 110 & 100 \underset{\mathrm{f} 1(\mathrm{ppm})}{90}\end{array}$ 
(R)-N-(2-Cyano-1-(4-chlorophenyl)ethyl)-P,P-diphenylphosphinic amide (2f):

${ }^{1} \mathbf{H}$ NMR $\left(400 \mathrm{MHz}, \mathrm{CDCl}_{3}\right)$

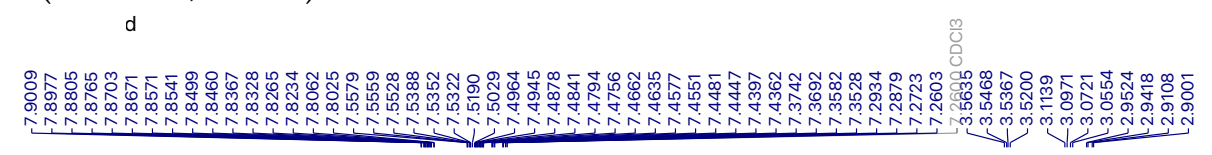<smiles>N#CCC(NPO)c1ccc(Cl)cc1</smiles>

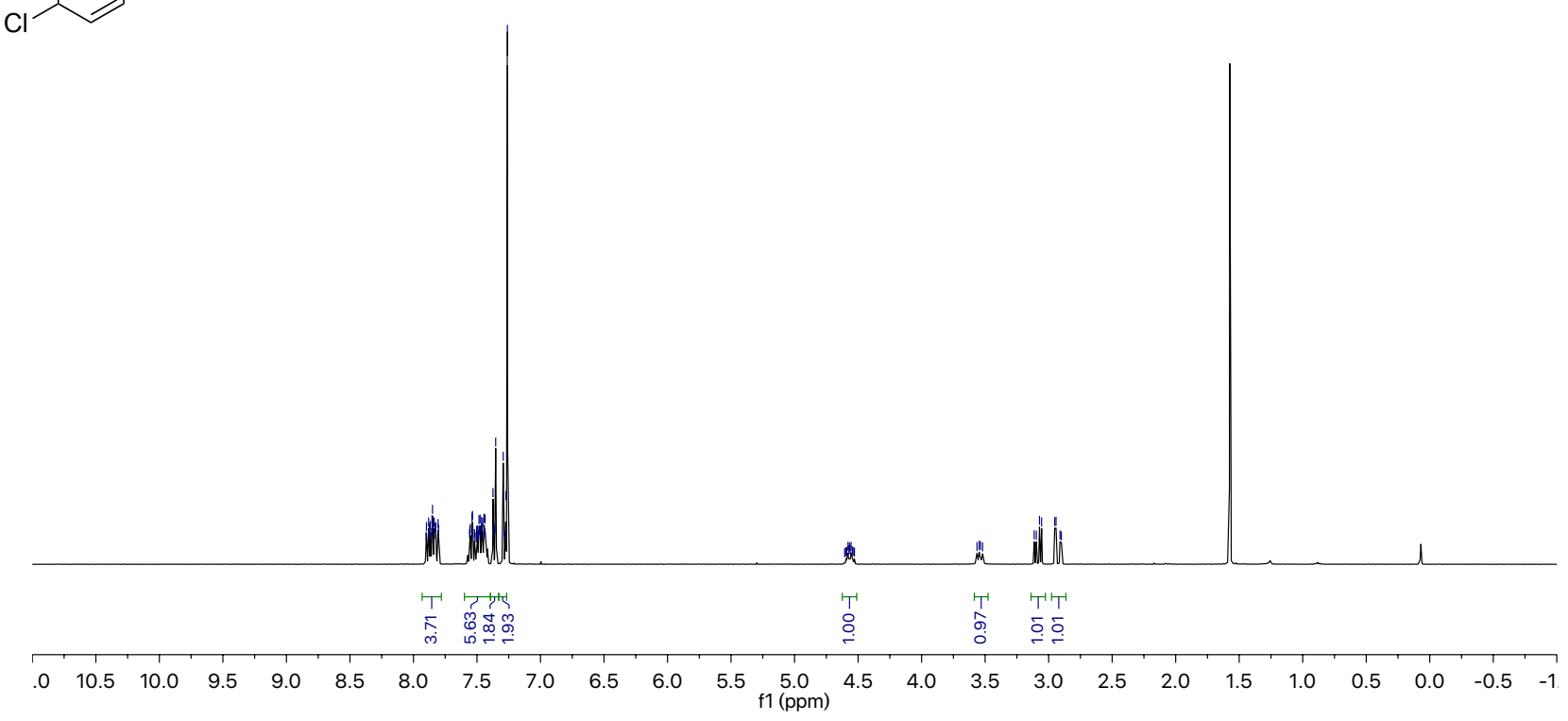

${ }^{13} \mathrm{C}$ NMR $\left(100 \mathrm{MHz}, \mathrm{CDCl}_{3}\right)$

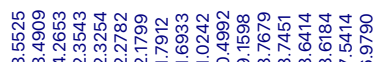

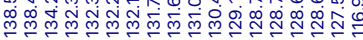
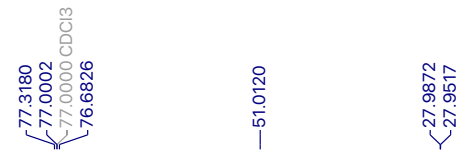<smiles>N#CCC(NPO)c1ccc(Cl)cc1</smiles>

$\begin{array}{llllllllll}190 & 180 & 170 & 160 & 150 & 140 & 130 & 120 & 110 & 100 \underset{\mathrm{f} 1(\mathrm{ppm})}{90}\end{array}$ 
(R)-N-(2-Cyano-1-(4-bromophenyl)ethyl)-P,P-diphenylphosphinic amide (2g):

${ }^{1} \mathrm{H}$ NMR $\left(400 \mathrm{MHz}, \mathrm{CDCl}_{3}\right)$

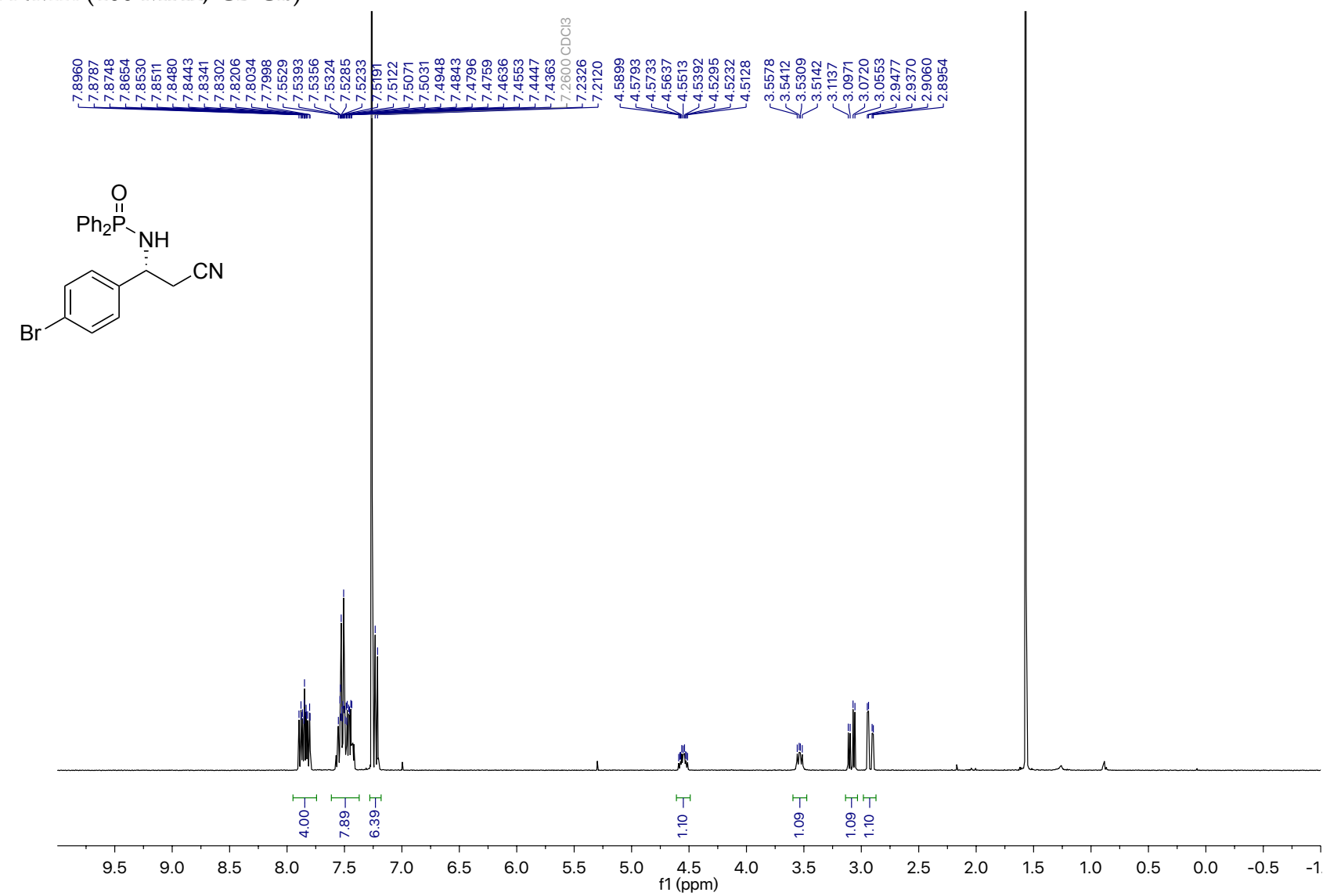

${ }^{13} \mathrm{C}$ NMR $\left(100 \mathrm{MHz}, \mathrm{CDCl}_{3}\right)$

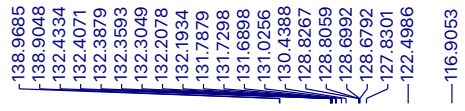<smiles>N#CCCc1ccc(Br)cc1NP(=O)(O)O</smiles>

$\begin{array}{llllllllll}190 & 180 & 170 & 160 & 150 & 140 & 130 & 120 & 110 & \begin{array}{c}100 \\ \mathrm{f} 1(\mathrm{ppm})\end{array}\end{array}$$$
\mathrm{Br}^{2}
$$

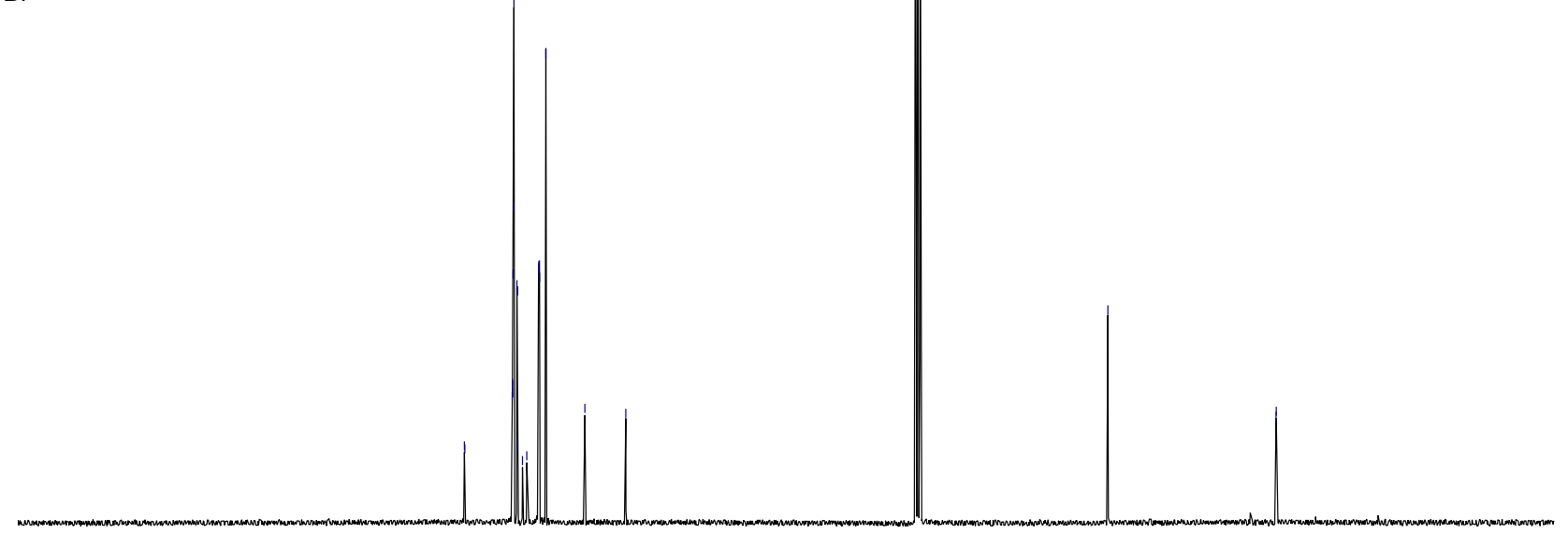


(R)-N-(2-Cyano-1-(4-(trifluoromethyl)phenyl)ethyl)- $P, P$-diphenylphosphinic amide (2h):

${ }^{1}$ H NMR $\left(400 \mathrm{MHz}, \mathrm{CDCl}_{3}\right)$

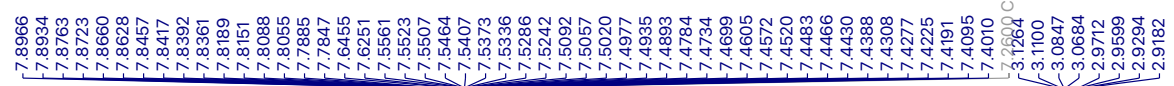

CN

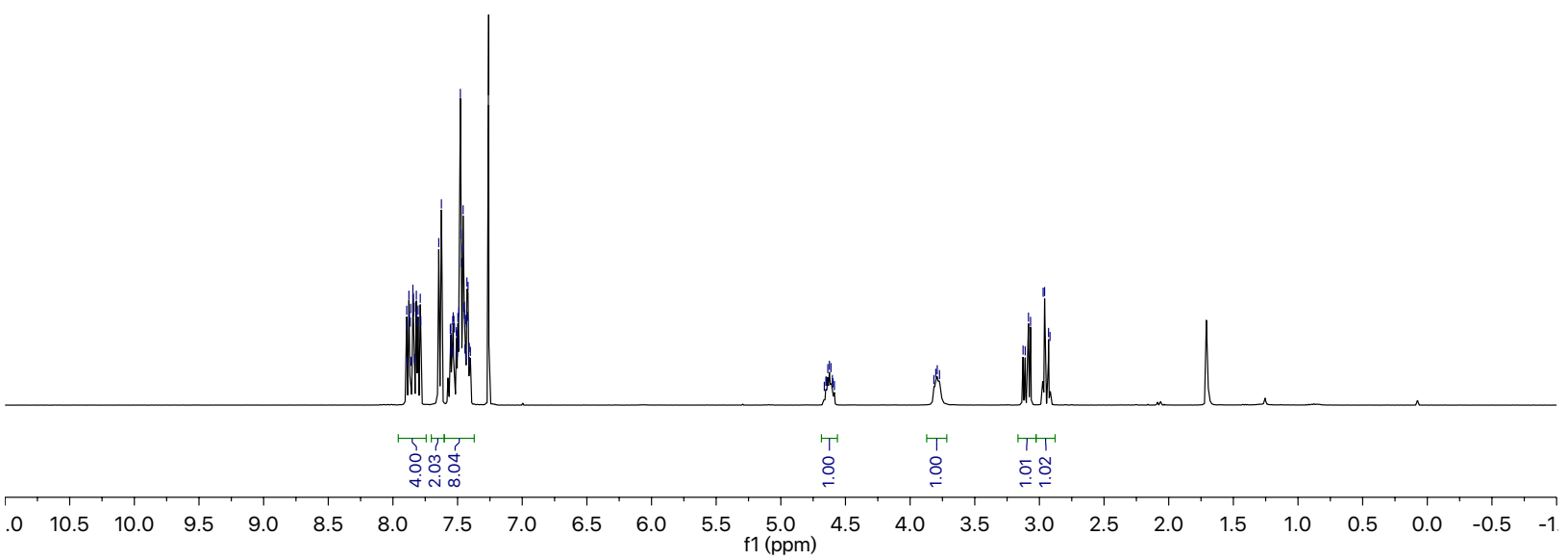

${ }^{13} \mathrm{C} \mathrm{NMR}\left(100 \mathrm{MHz}, \mathrm{CDCl}_{3}\right)$

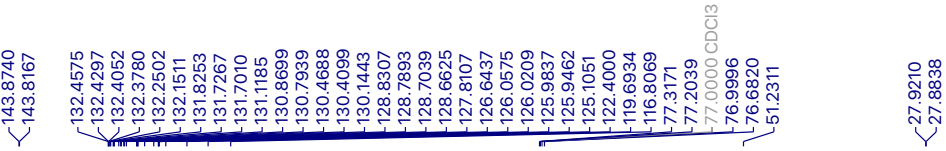<smiles>N#CC[C@H](NP=O)c1ccc(C(F)(F)F)cc1</smiles>

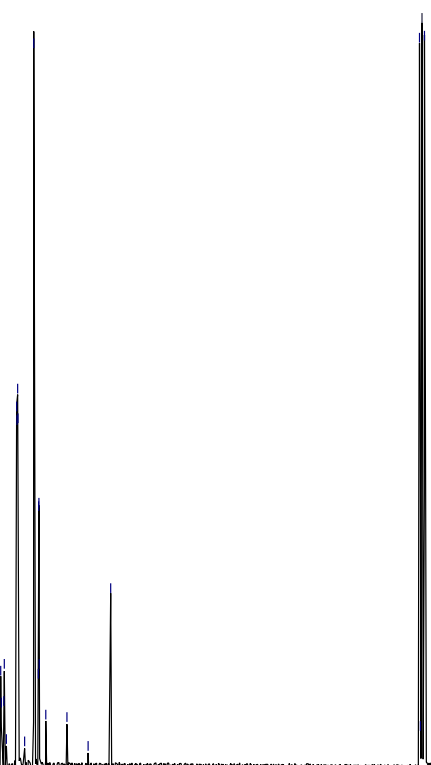

$\mathrm{F}_{3}$

$\begin{array}{llllllllll}190 & 180 & 170 & 160 & 150 & 140 & 130 & 120 & 110 & \begin{array}{c}100 \\ \mathrm{f} 1(\mathrm{ppm})\end{array}\end{array}$ 
(R)-N-(2-Cyano-1-(4-cyanophenyl)ethyl)-P,P-diphenylphosphinic amide (2i):

${ }^{1} \mathbf{H}$ NMR $\left(400 \mathrm{MHz}, \mathrm{CDCl}_{3}\right)$

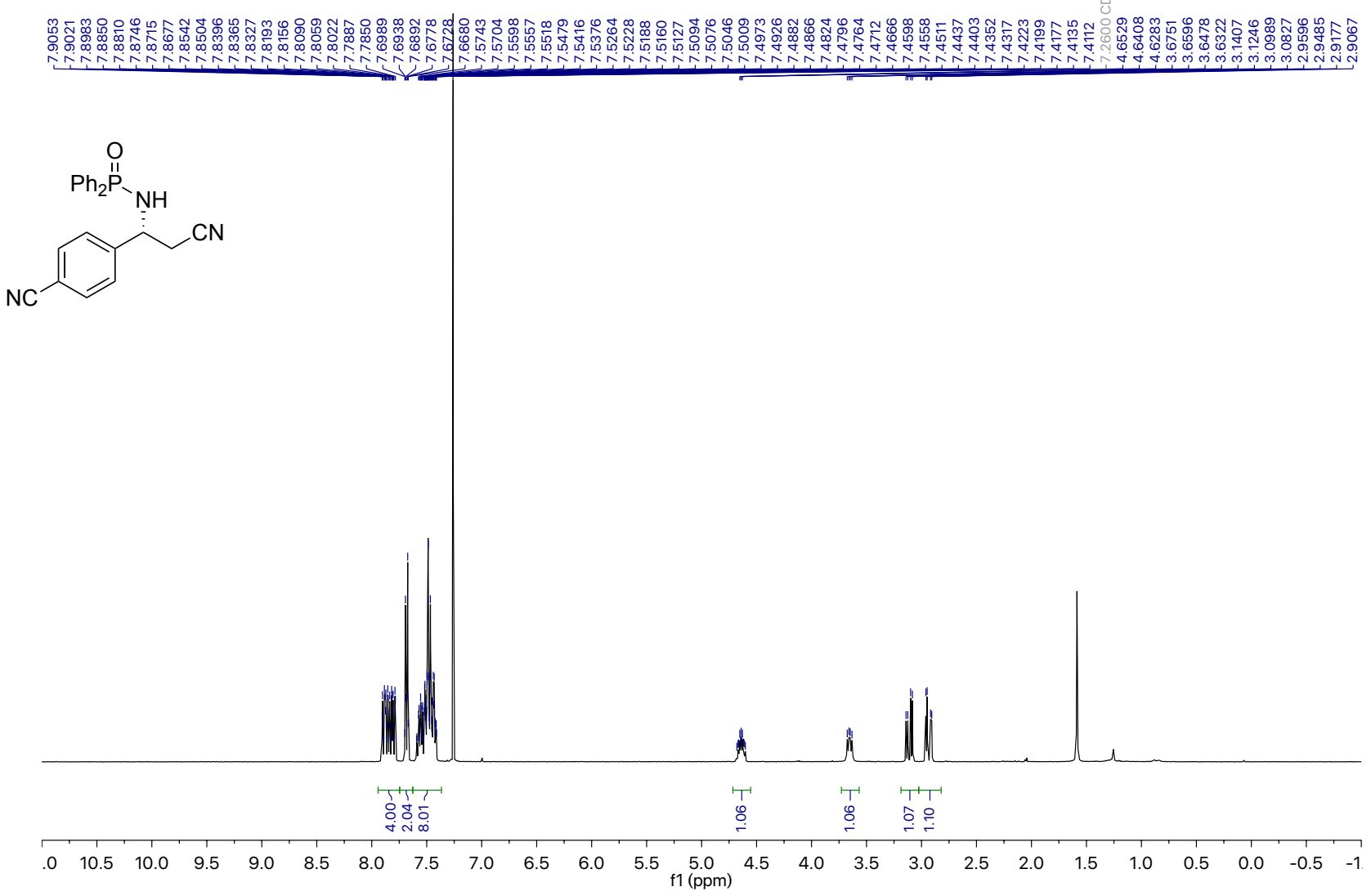

${ }^{13} \mathrm{C}$ NMR $\left(100 \mathrm{MHz}, \mathrm{CDCl}_{3}\right)$

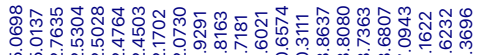

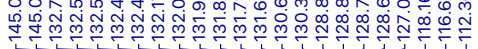<smiles>N#CCCc1ccc(NPO)cc1</smiles>

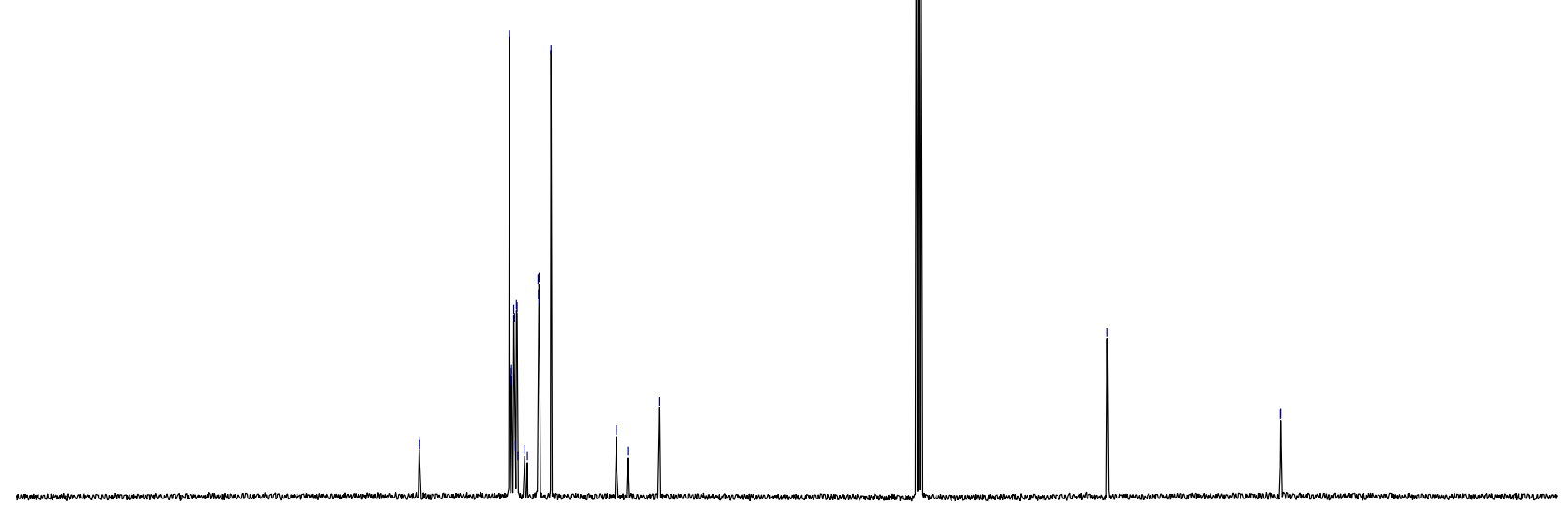

$\begin{array}{llllllllll}190 & 180 & 170 & 160 & 150 & 140 & 130 & 120 & 110 & 100 \underset{\mathrm{f} 1(\mathrm{ppm})}{90}\end{array}$

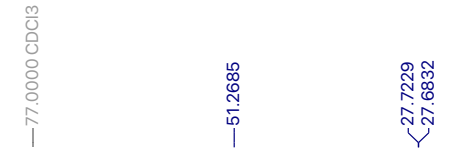


(R)-N-(2-Cyano-1-(furan-2-yl)ethyl)-P,P-diphenylphosphinic amide (2j):

1 H NMR (400 MHz, $\left.\mathrm{CDCl}_{3}\right)$

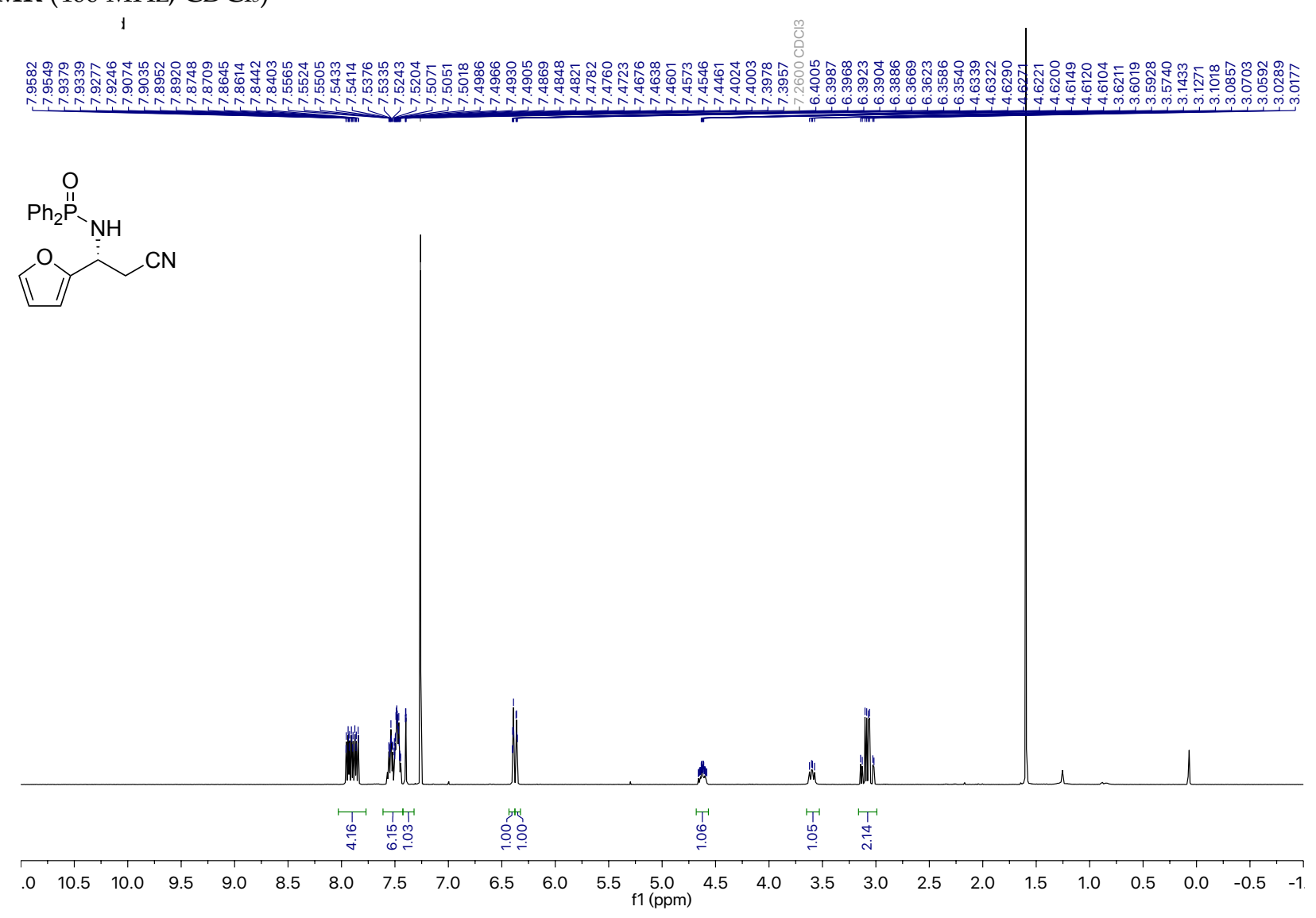

${ }^{13} \mathrm{C}$ NMR $\left(100 \mathrm{MHz}, \mathrm{CDCl}_{3}\right)$

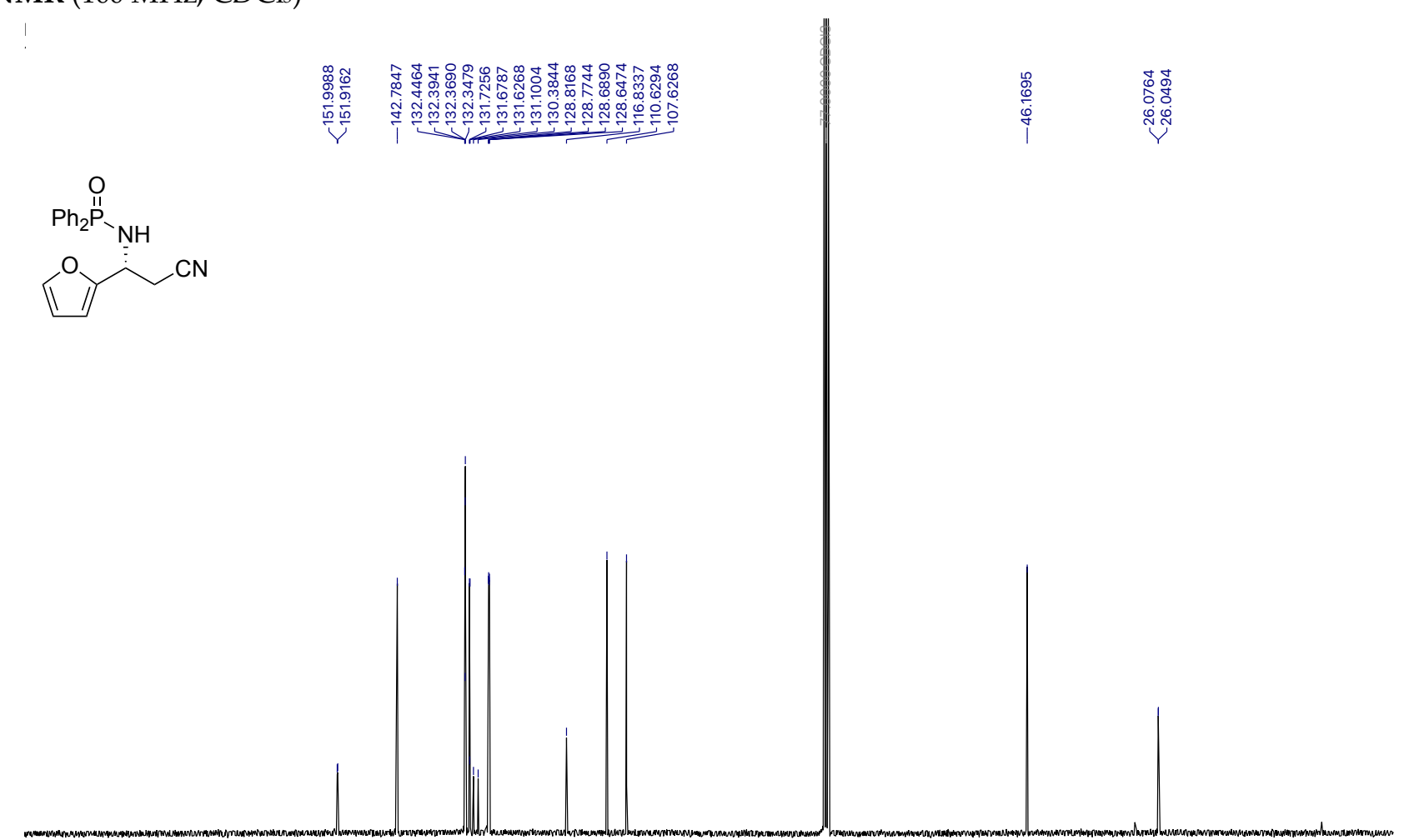

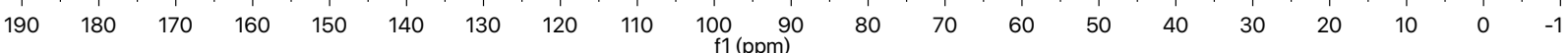


(R)-N-(2-Cyano-1-(thiophen-2-yl)ethyl)-P,P-diphenylphosphinic amide (2k):

${ }^{1} \mathbf{H}$ NMR $\left(400 \mathrm{MHz}, \mathrm{CDCl}_{3}\right)$

d

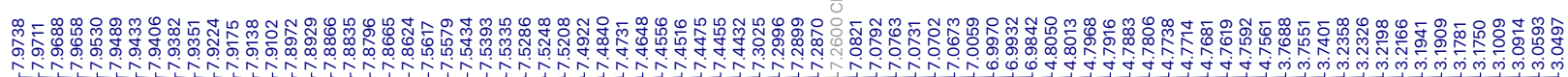<smiles>N#CCC(NP(=O)=O)c1cccs1</smiles>

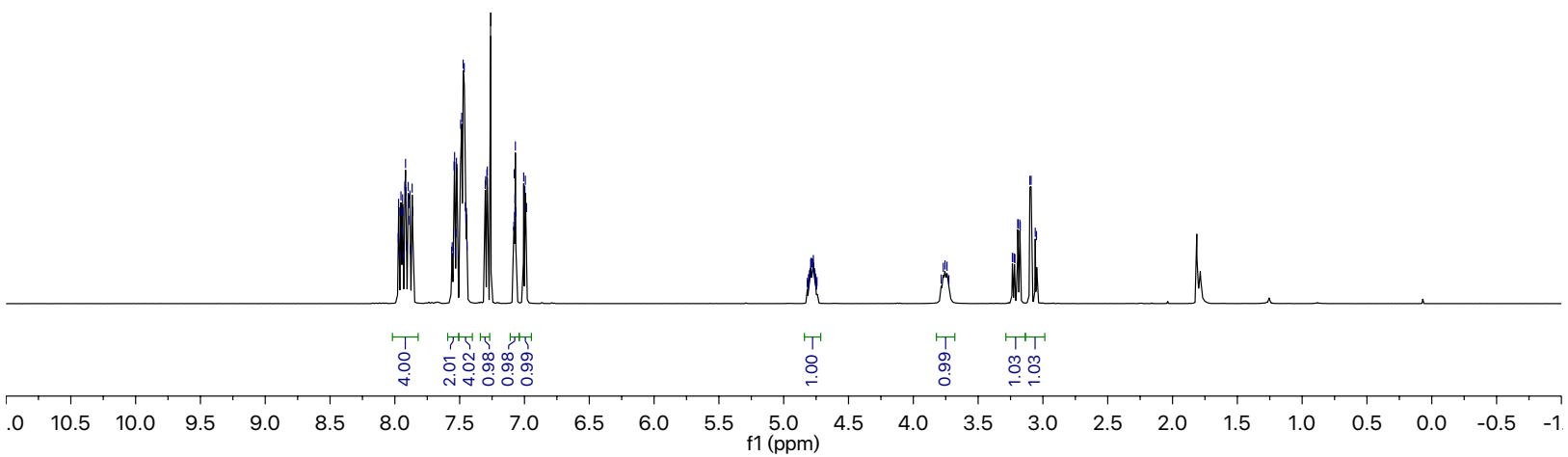

${ }^{13} \mathrm{C}$ NMR $\left(100 \mathrm{MHz}, \mathrm{CDCl}_{3}\right)$

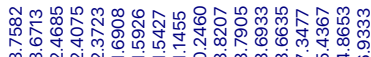

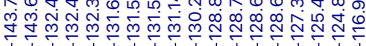<smiles>N#CCC(NP(=O)(O)c1cccs1)c1ccccc1</smiles>

$\begin{array}{llllllllll}190 & 180 & 170 & 160 & 150 & 140 & 130 & 120 & 110 & \begin{array}{c}100 \\ \mathrm{f} 1(\mathrm{ppm})\end{array}\end{array}$
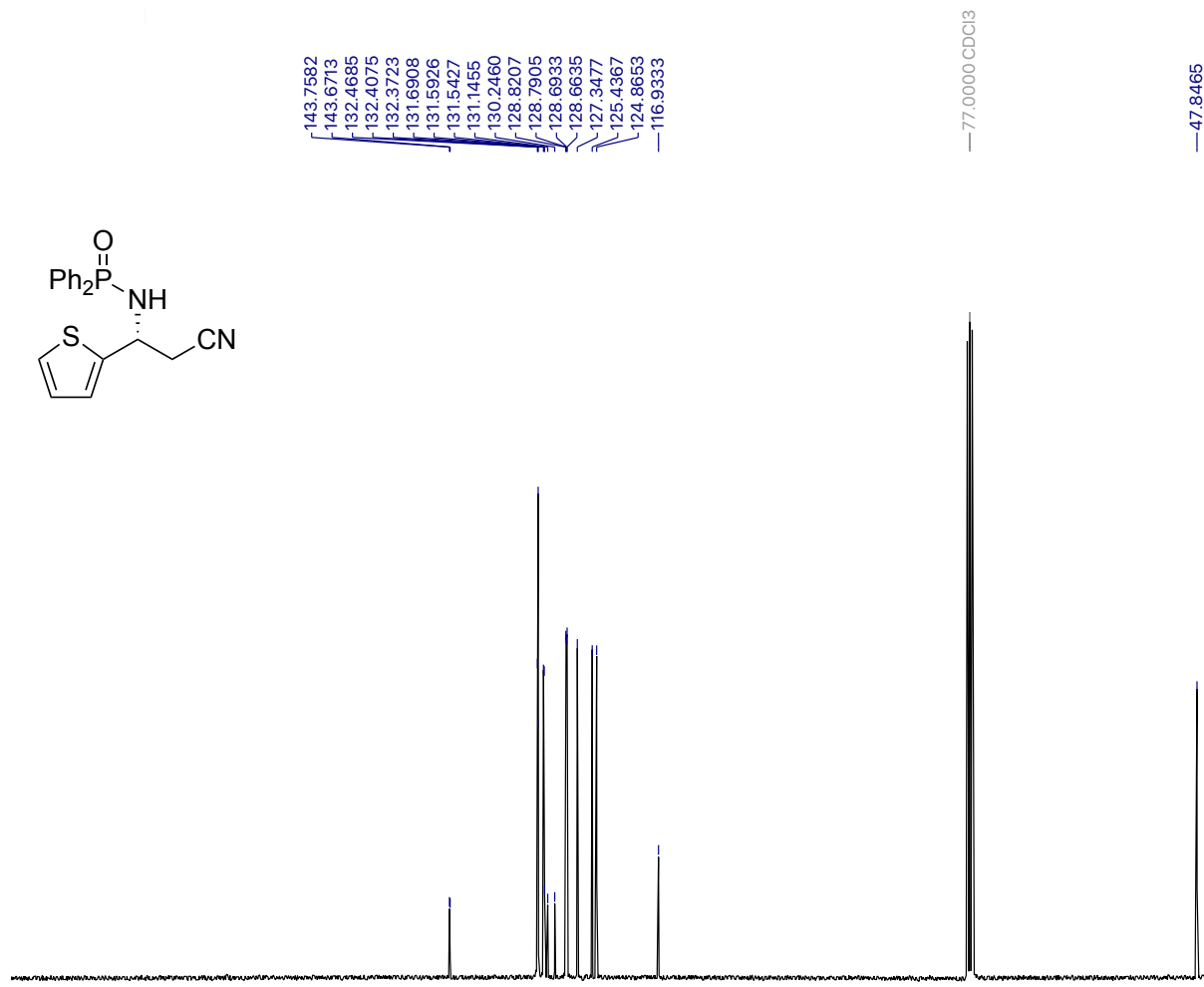
$(R, E)-N$-(1-Cyano-4-phenylbut-3-en-2-yl)-P,P-diphenylphosphinic amide (21):

${ }_{1}^{1} \mathbf{H}$ NMR (400 MHz, $\left.\mathrm{CDCl}_{3}\right)$
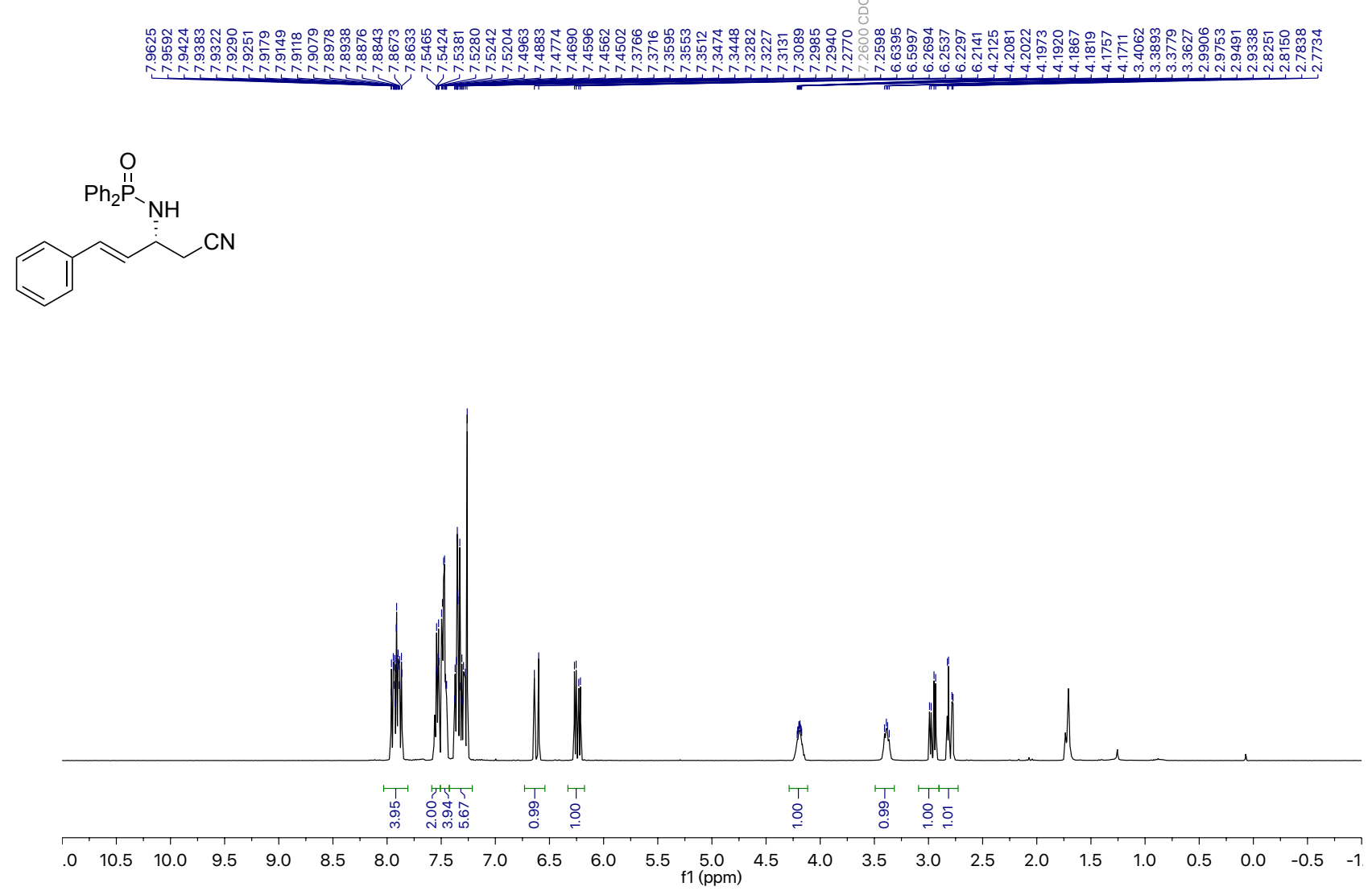

${ }^{13} \mathrm{C}$ NMR $\left(100 \mathrm{MHz}, \mathrm{CDCl}_{3}\right)$

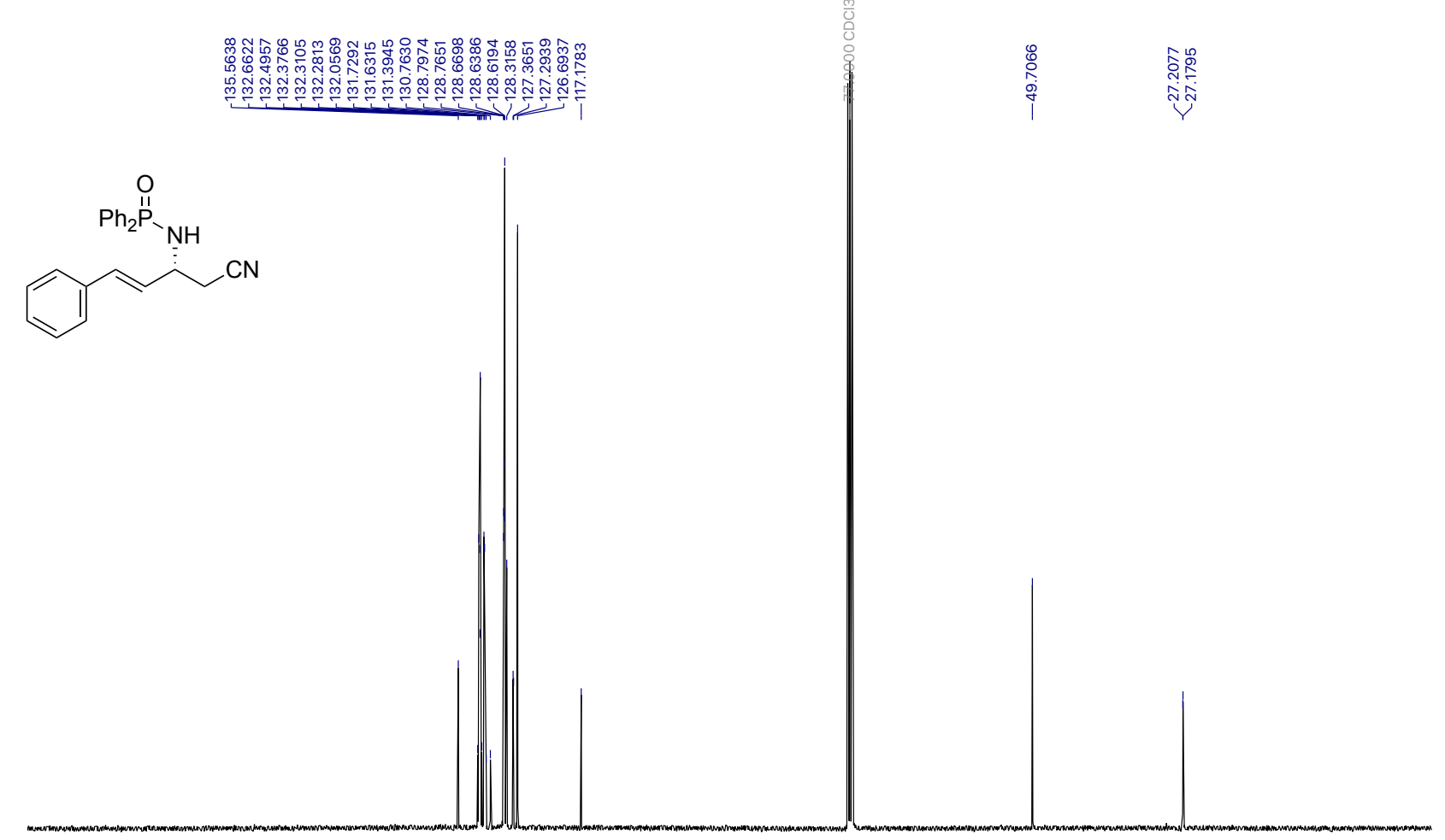

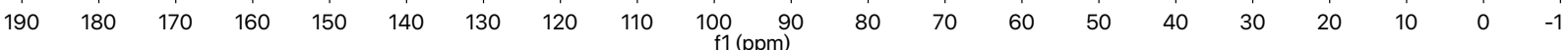


(R)-3-((Diphenylphosphoryl)amino)-3-phenylpropanoic acid (4):

${ }^{1}$ H NMR $\left(400 \mathrm{MHz}, \mathrm{CDCl}_{3}\right)$

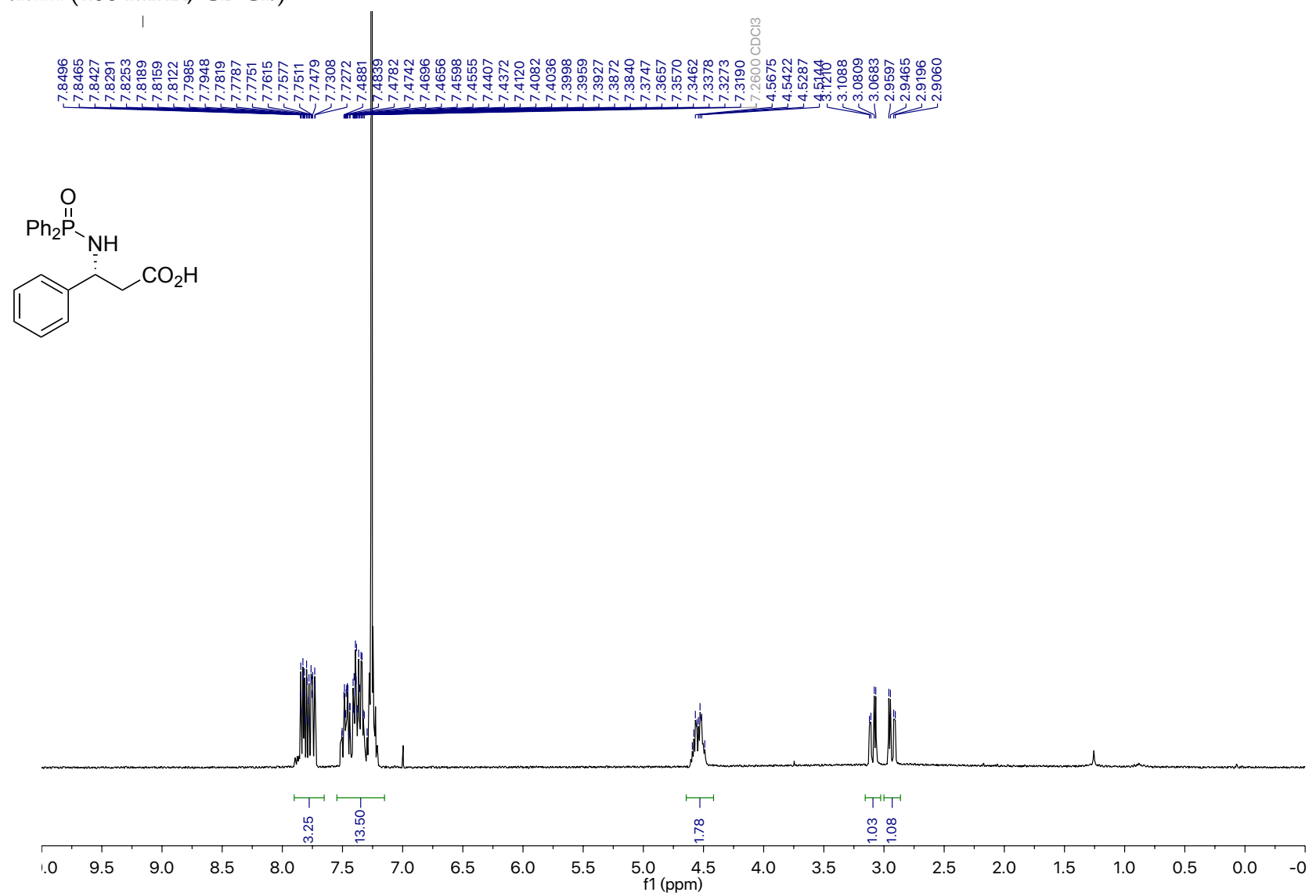

${ }^{13} \mathrm{C}$ NMR $\left(100 \mathrm{MHz}, \mathrm{CDCl}_{3}\right)$

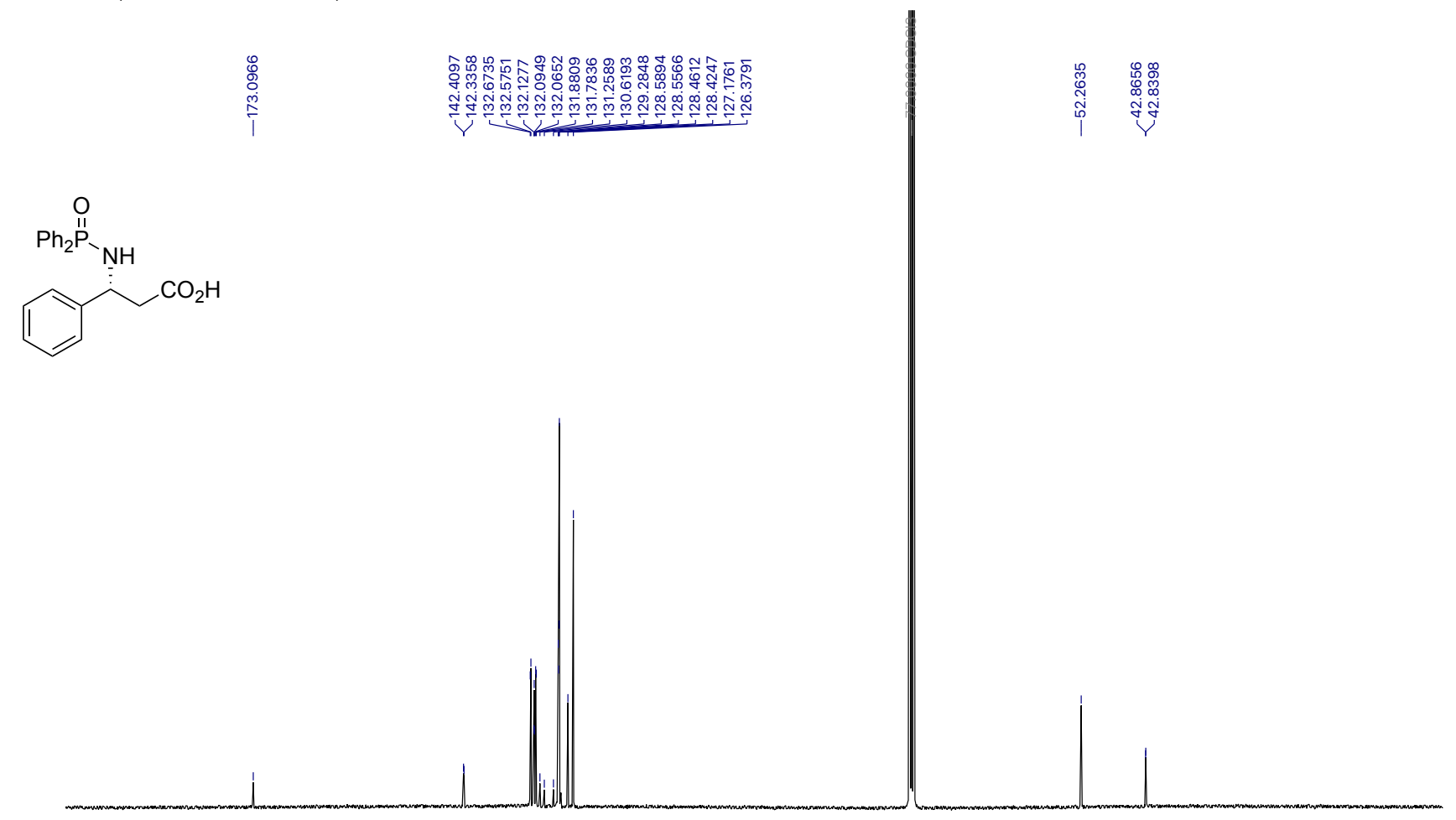

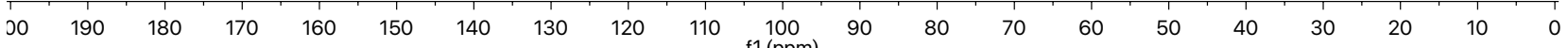


(R)- $N$-(3-Amino-1-phenylpropyl)- $P, P$-diphenylphosphinic amide (5):

${ }^{1} \mathbf{H}$ NMR (400 MHz, $\left.\mathrm{CDCl}_{3}\right)$

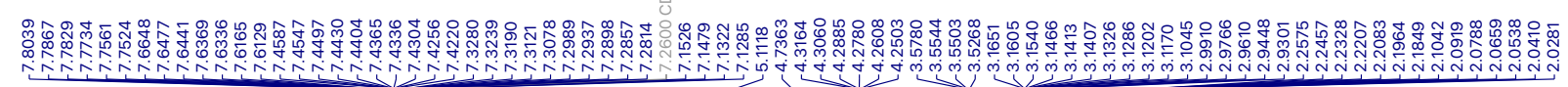<smiles>NCCC(NP=O)c1ccccc1</smiles>

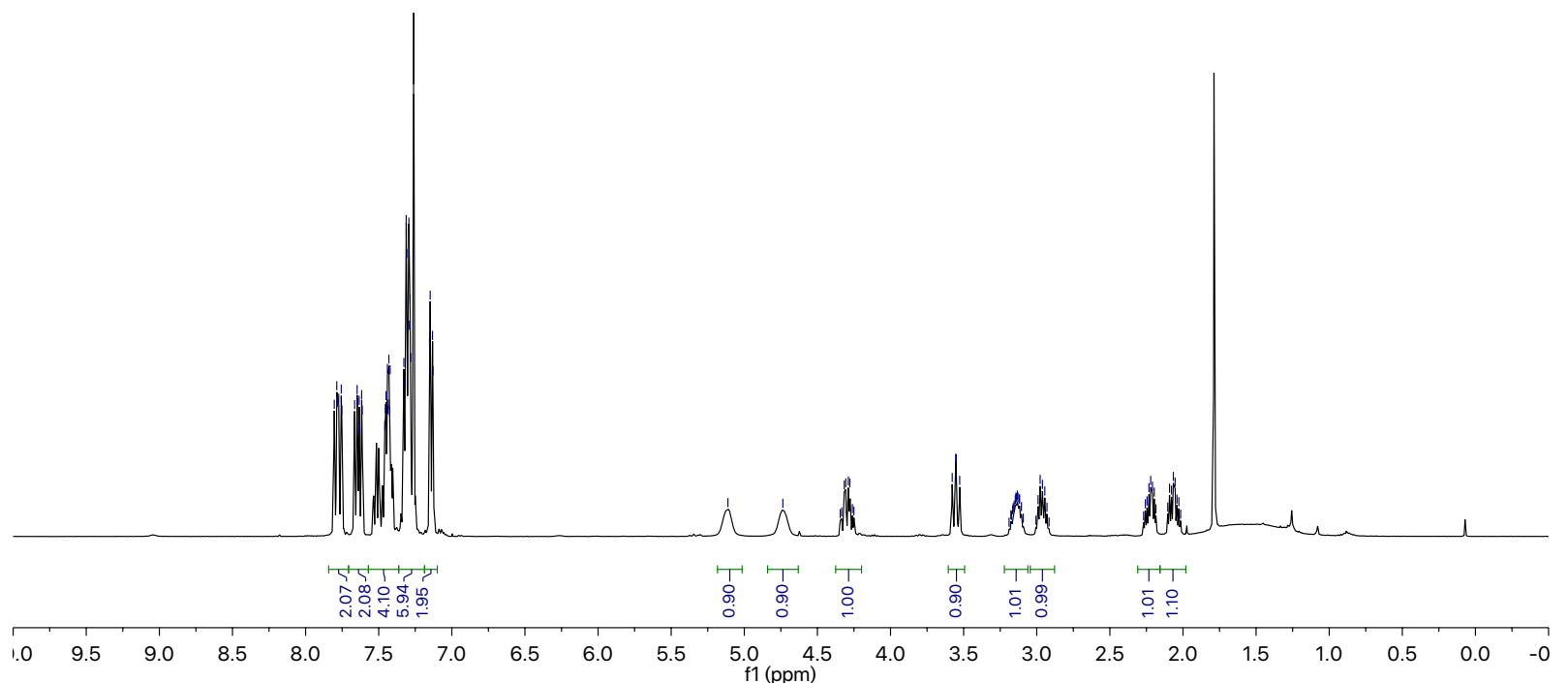

${ }^{13} \mathrm{C}$ NMR (100 MHz, $\left.\mathrm{CDCl}_{3}\right)$<smiles>NCCC(NP=O)c1ccccc1</smiles>

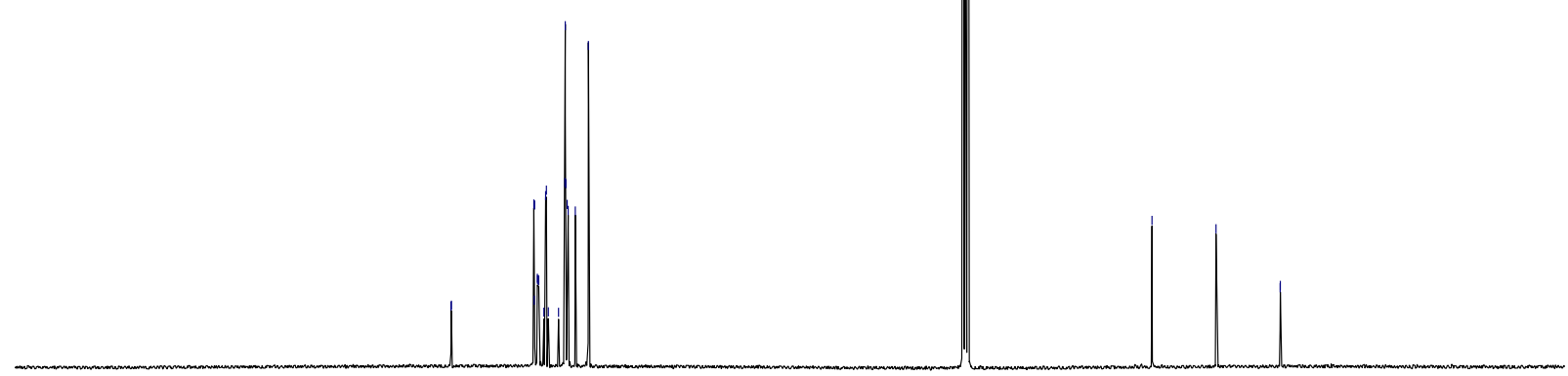

